\title{
$/ *$
}

tracer20.c

Source code for the TRACER contaminated sediment transport model, based on the CAESAR model.

This code and the sample data was prepared for GEOLOGY on 14/01/2003.

This code was developed by Tom Coulthard of the University of Wales Aberystwyth, U.K. More details may be found on http://WwW. coulthard.org.uk or email

T.Coulthard@aber.ac.uk

We (Tom Coulthard and the University of wales, Aberystwyth) accept no responsibility whatsoever for any predictions made or results obtained from this model. It is operated and results interpreted entirely at the users risk.

This code is free for you to use, but we ask that we are correctly acknowledged and referenced in any publications stemming from its use or development. We would also be gratefull if users could let us know how they get on with this code, and whether they made any modifications - so that we can keep on top of how things are progressing.

Many thanks, and good luck!

Tom Coulthard.

PS. MY aplolgies for the awfull coding methods used and any profanities that may exist within the code comments!

Notes on compiling.

I generally use this on Linux machines - compilex command gcc tracer $20 . \mathrm{c}-1 \mathrm{~m}-\mathrm{O}$ -o tracer20. exe

also tested on Compaq alpha (CC not GCC) and SGI machines. It uses fairly bog standard C - no graphcics etc. so should work on just about any stock C compiler.

*/

\#include "stdio.h"

\#include "math.h"

/*** reach $=$ lenght, $x$ section $=$ width, active=active layer thickness $* * /$

\#define reach 204

\#define xsection 887

\#define ACTIVE FACTOR 1

\#define TRUE 1

\#define FAISE 0

\#define ERODEFACTOR $0.05 / *$ normally $0.02 * /$

\#define DX 50

\#define root $70.71 / *$ must be equal to root $(2 \times \mathrm{DX}) * /$

\#define LIMIT 3

\#define MIN $Q 0.5 / *$ must be $0.001 *$ pow $(\mathrm{DX}, 2)$, since discovered 0.002

better..*/

\#define CREEP_RATE 0.0025

/** these all save me writing the code in every time **/ 


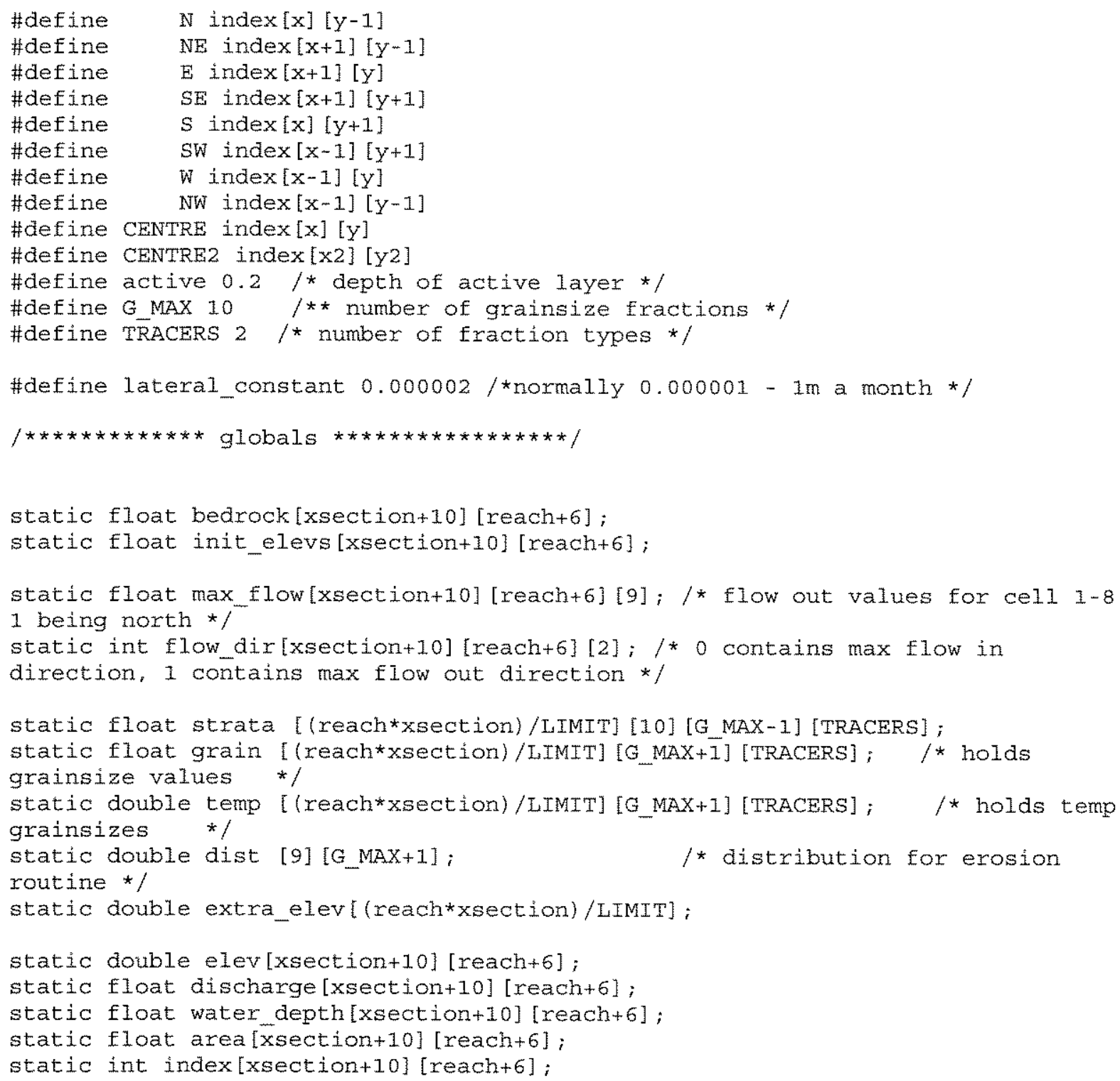

static int cross scan [xsection+1] [reach+3]; static int down scan [reach +1$][x$ section +3$]$;

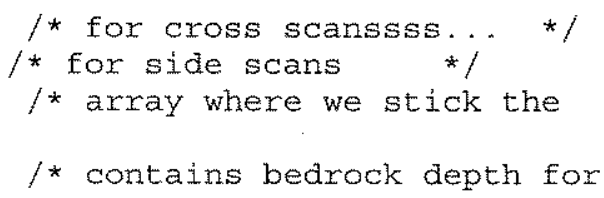




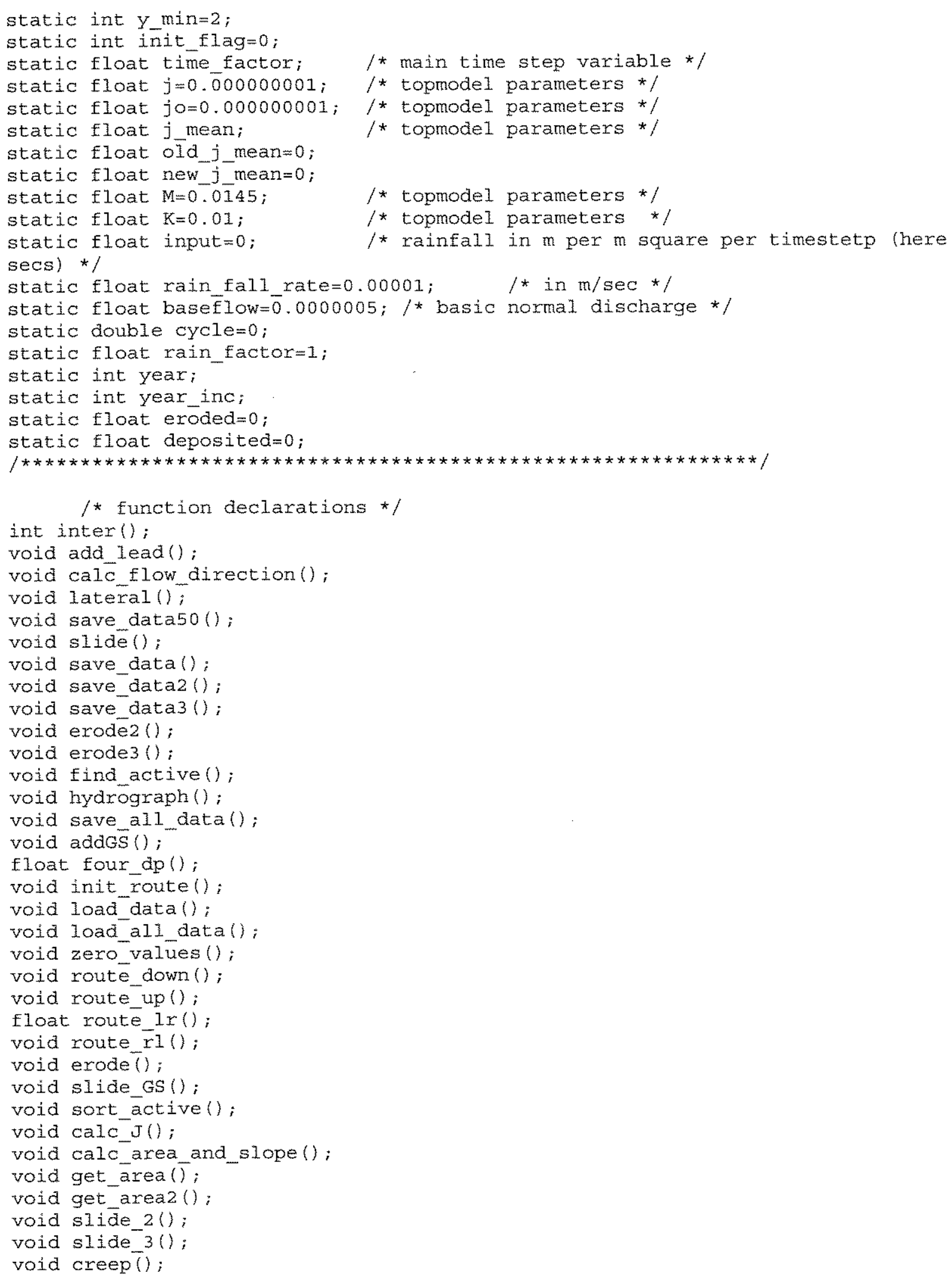




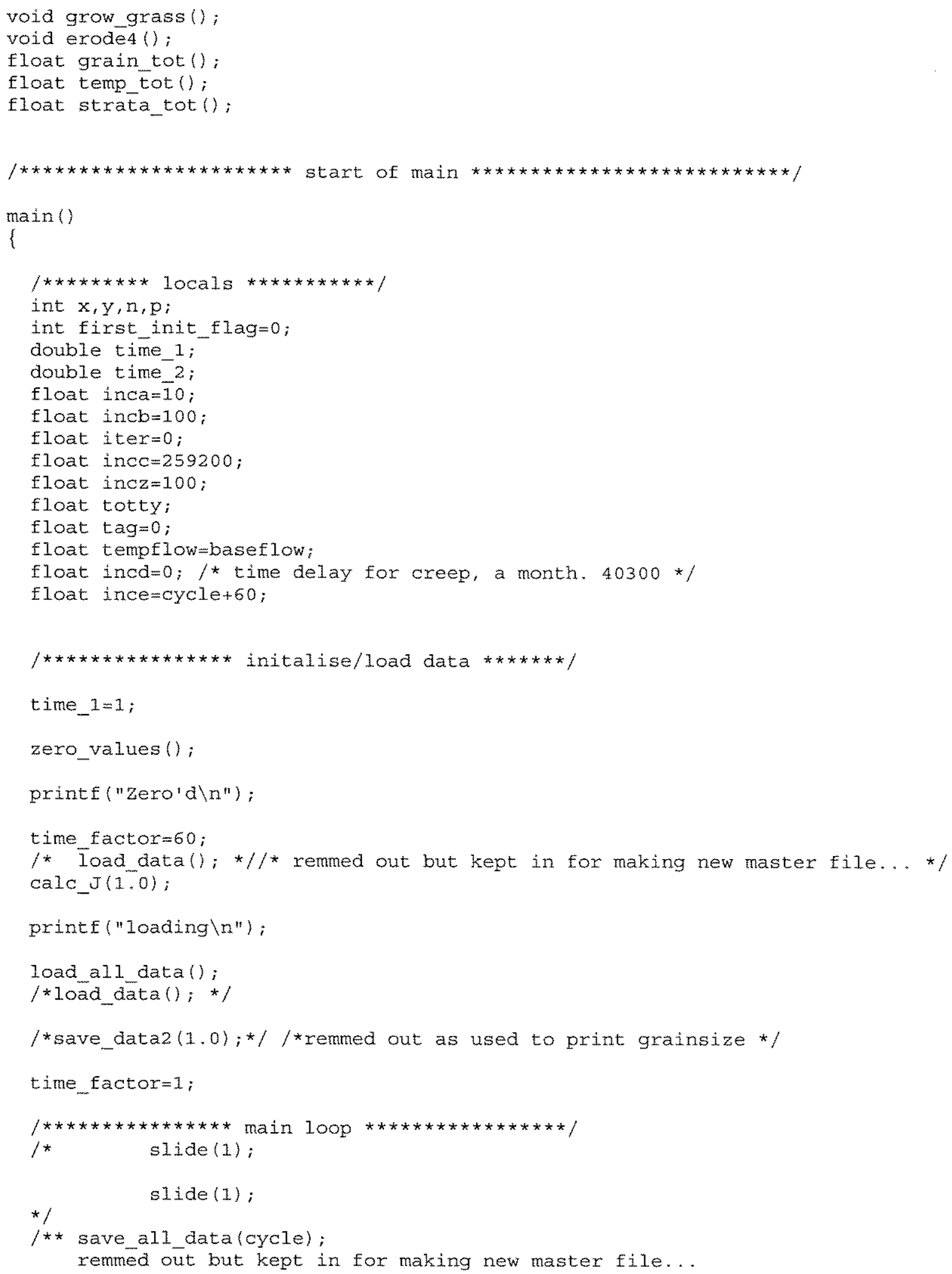




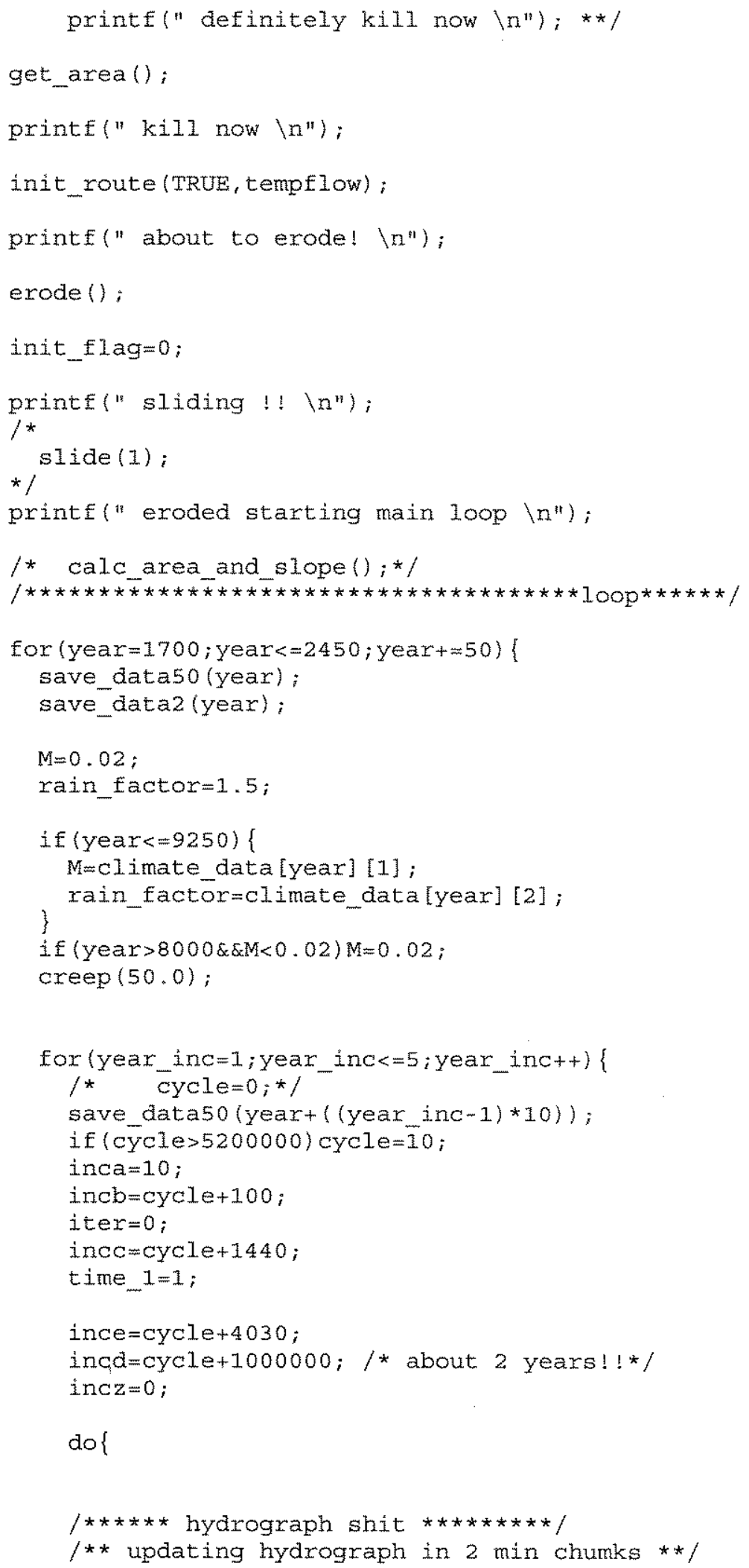




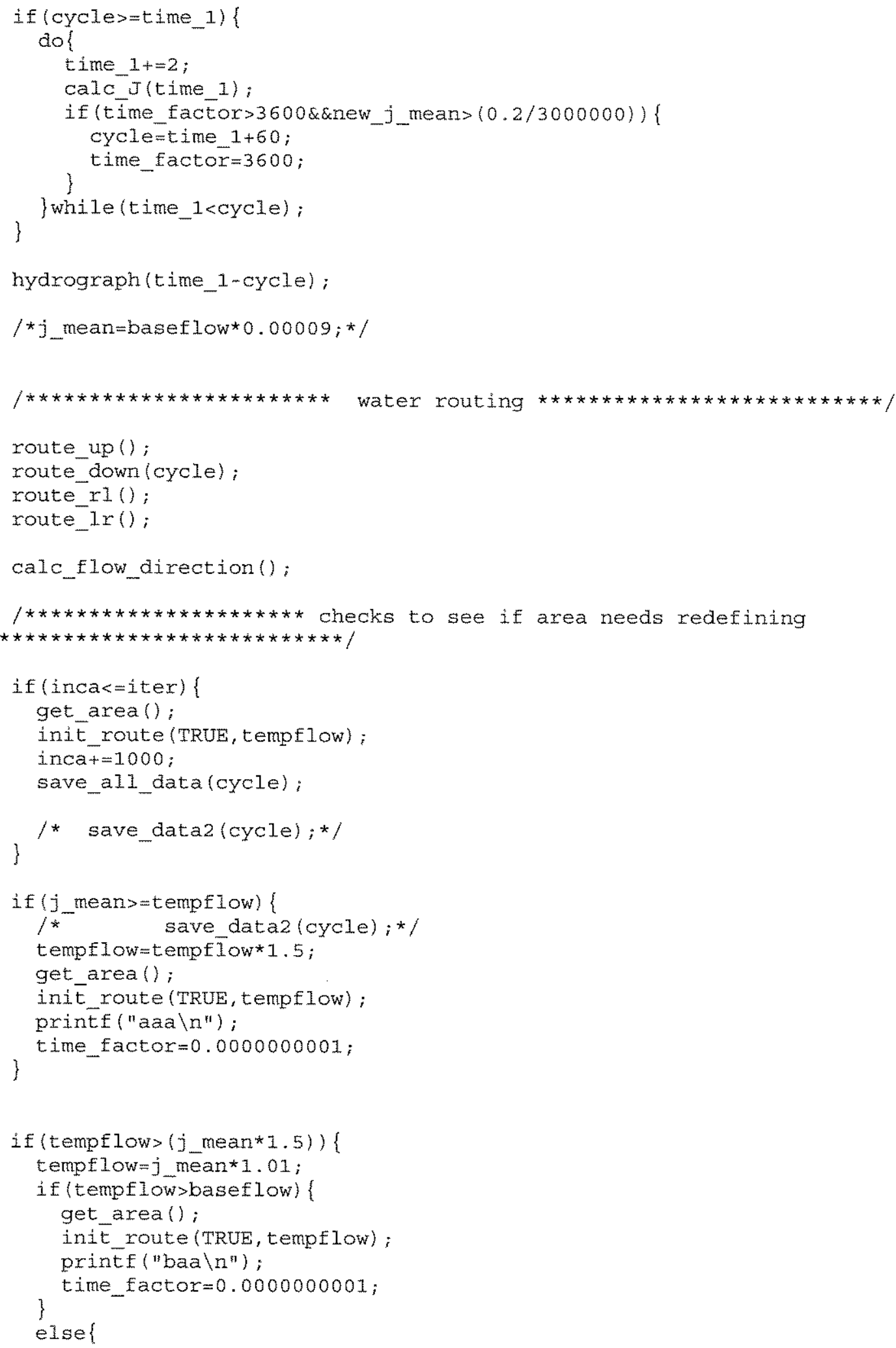




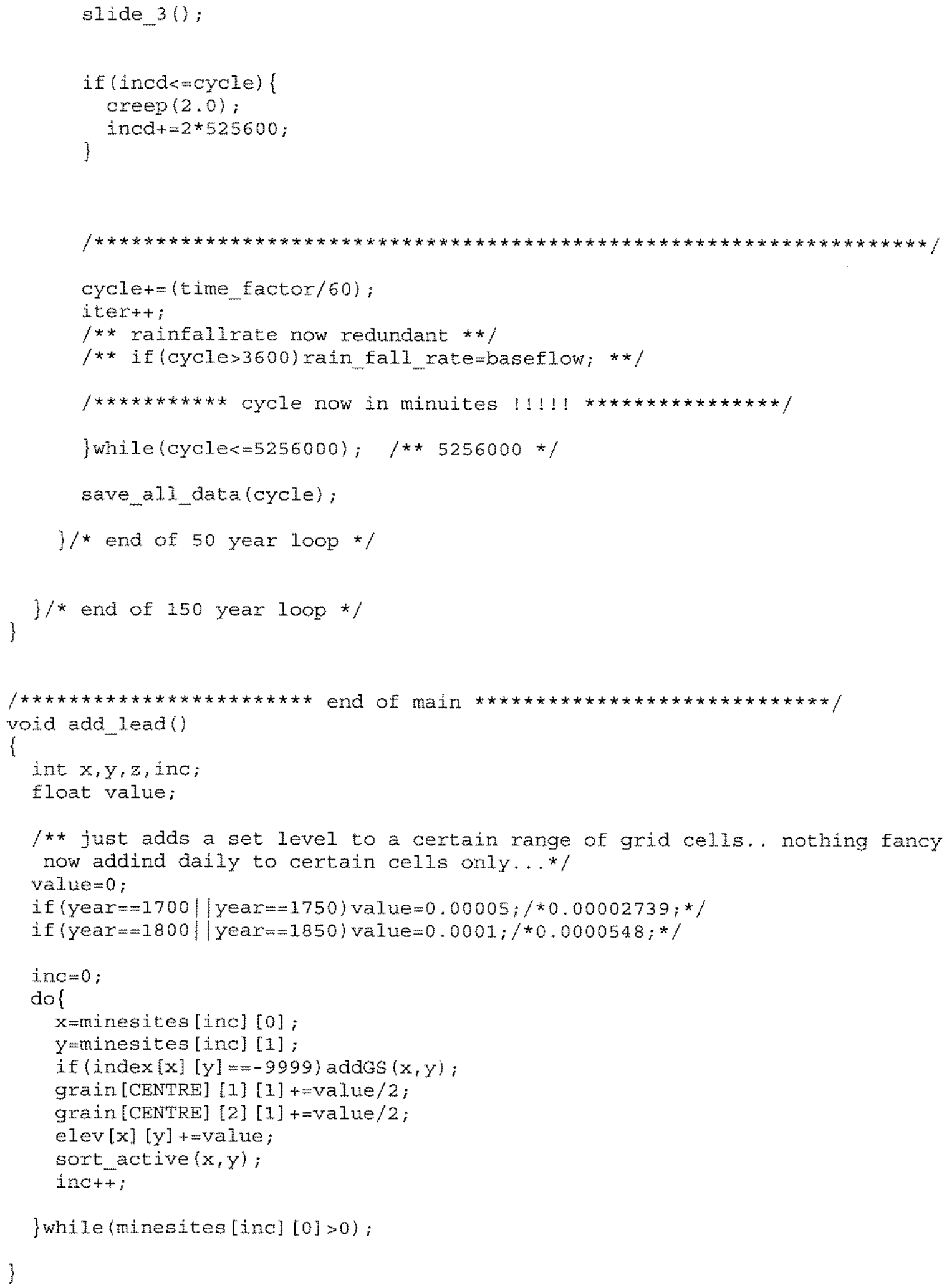




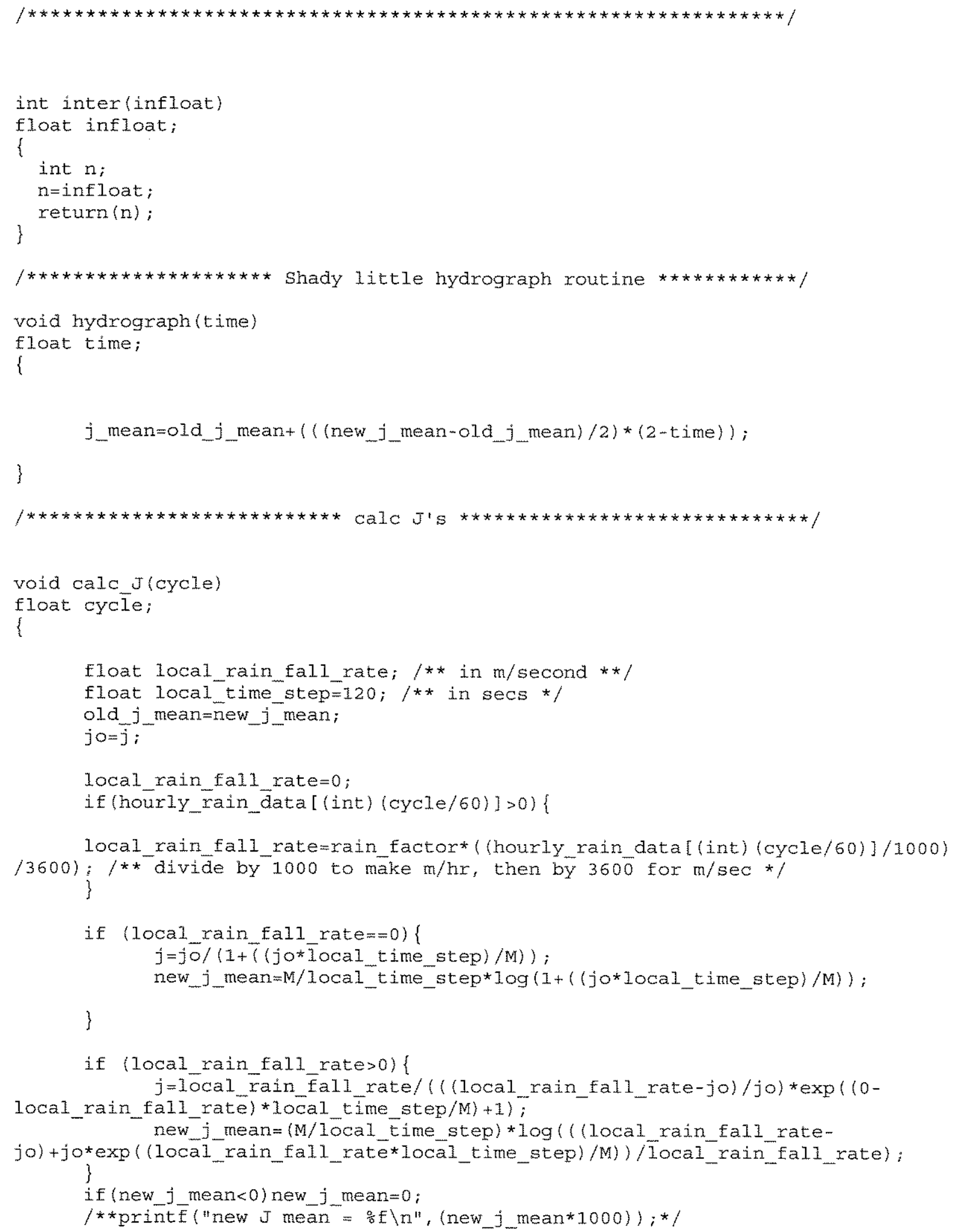




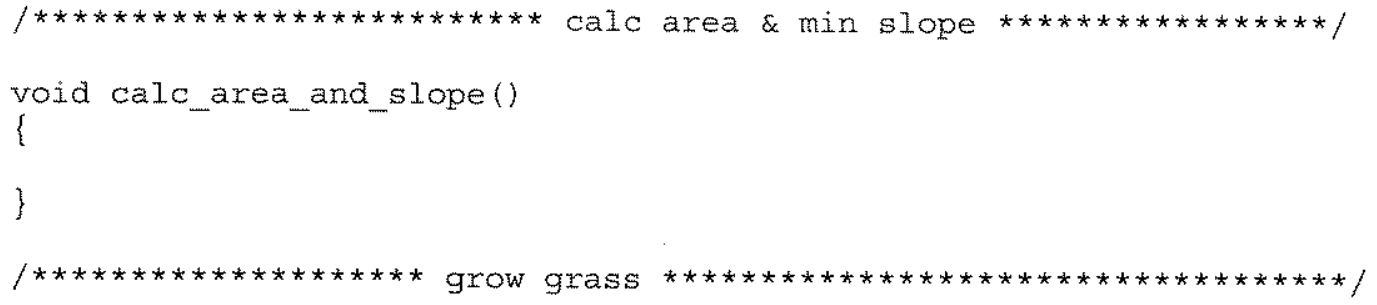




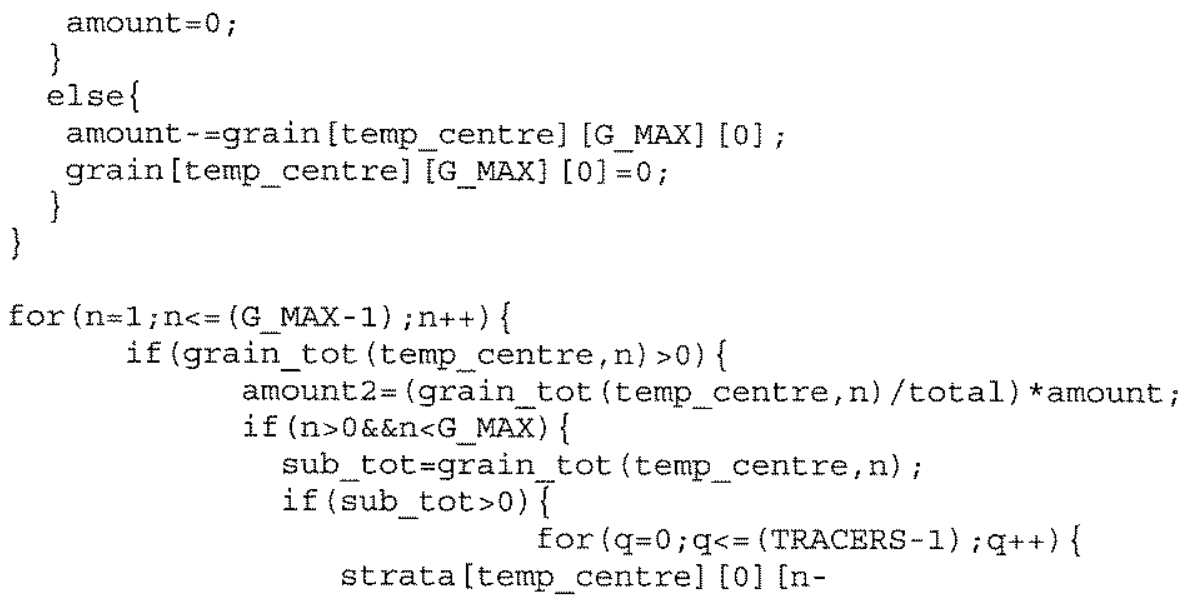

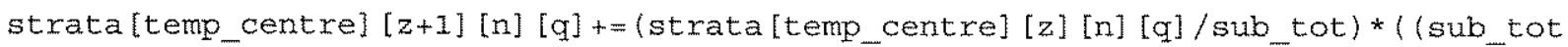
(tota12)*amount);

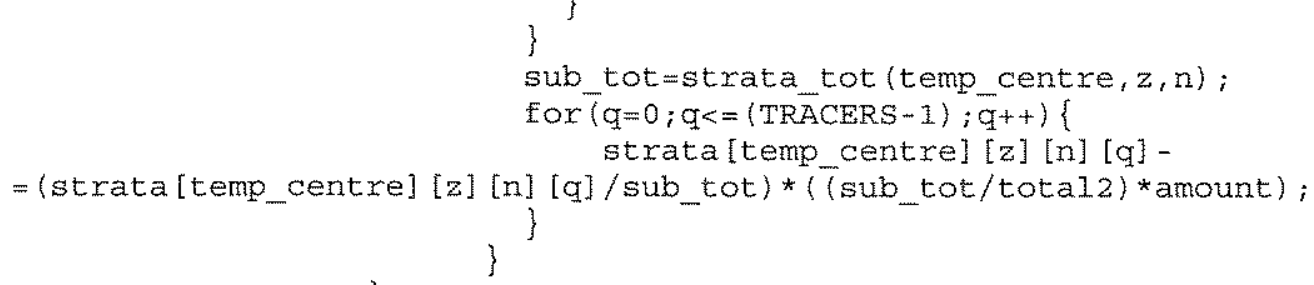




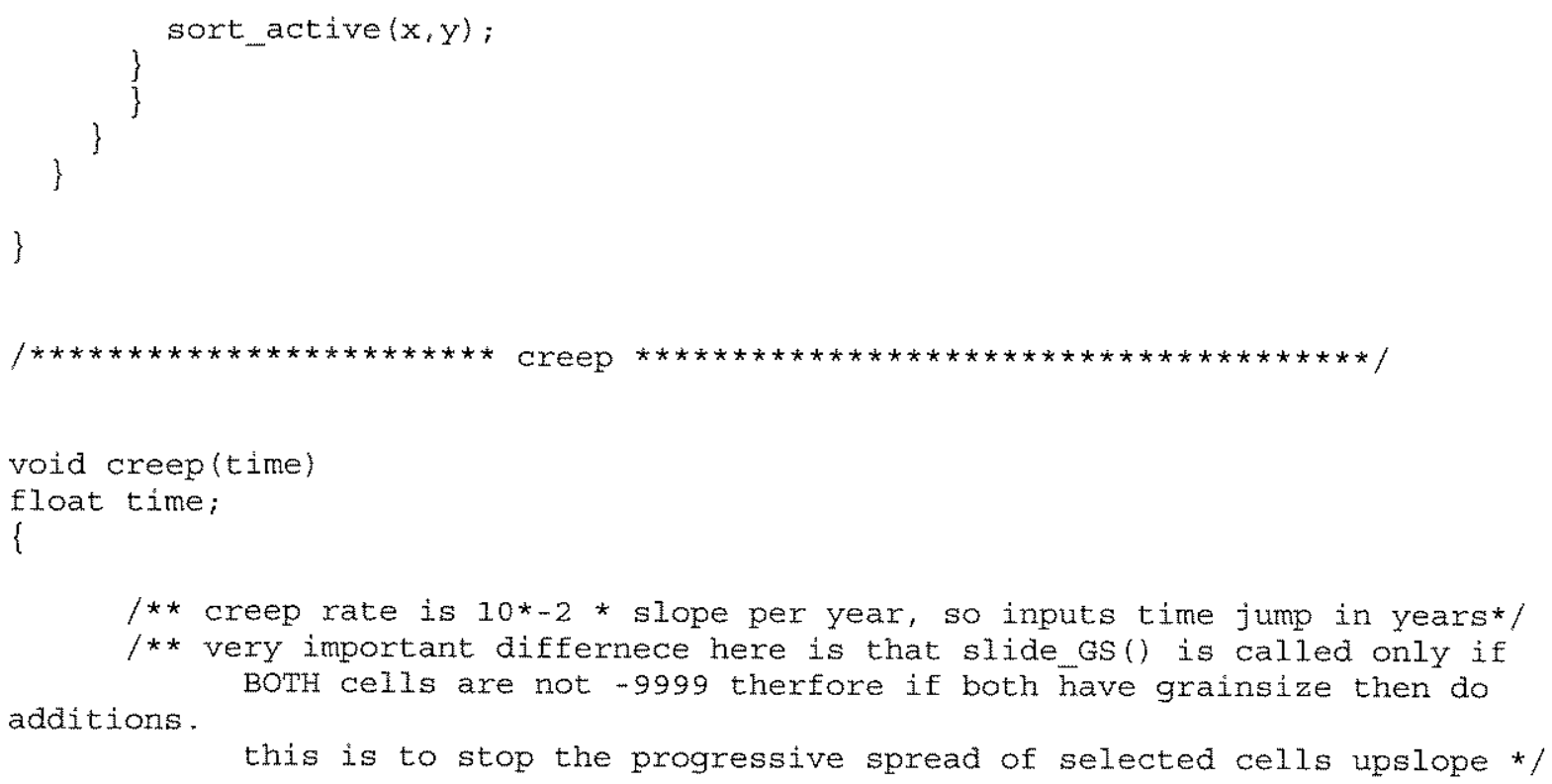




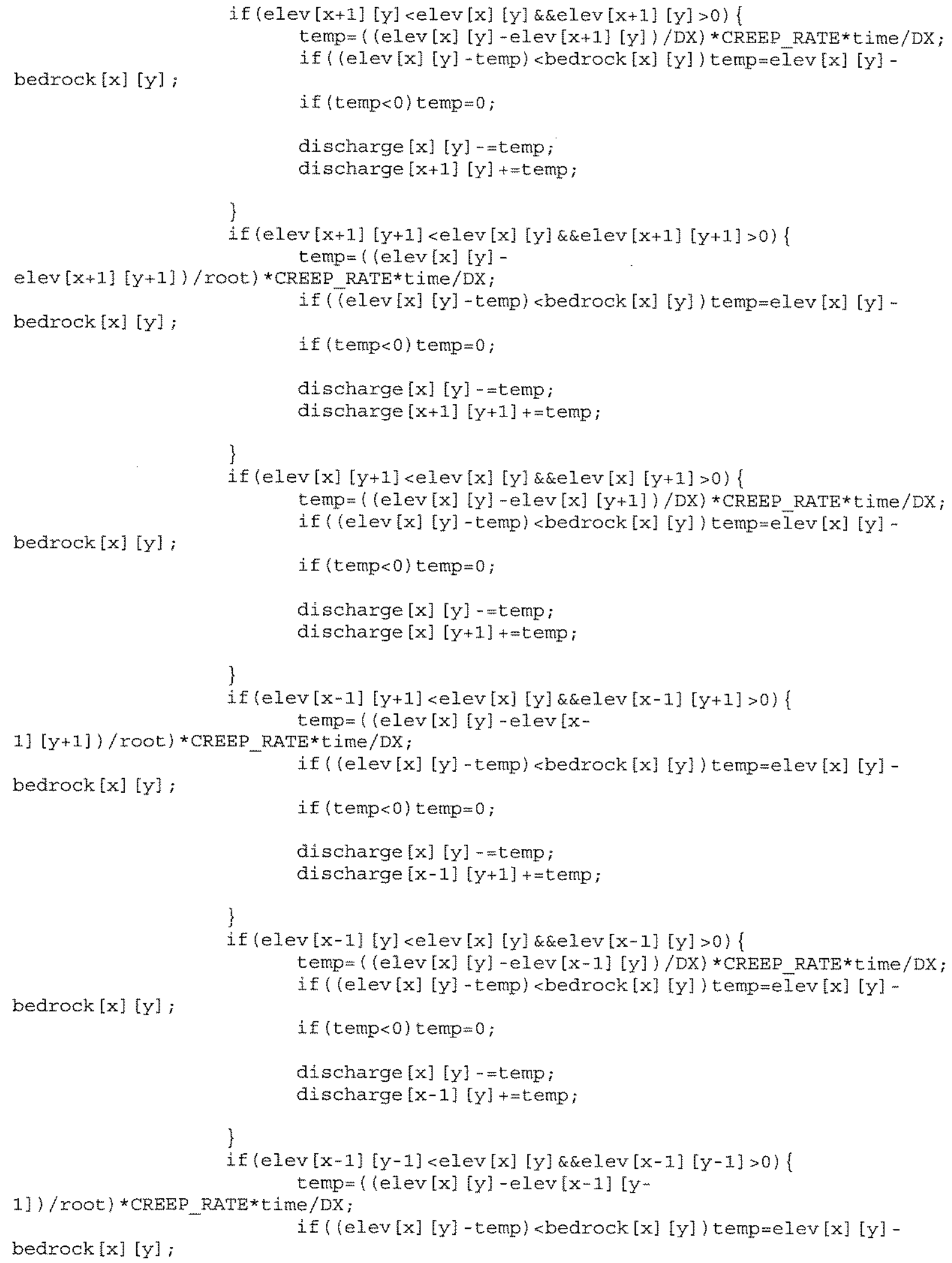




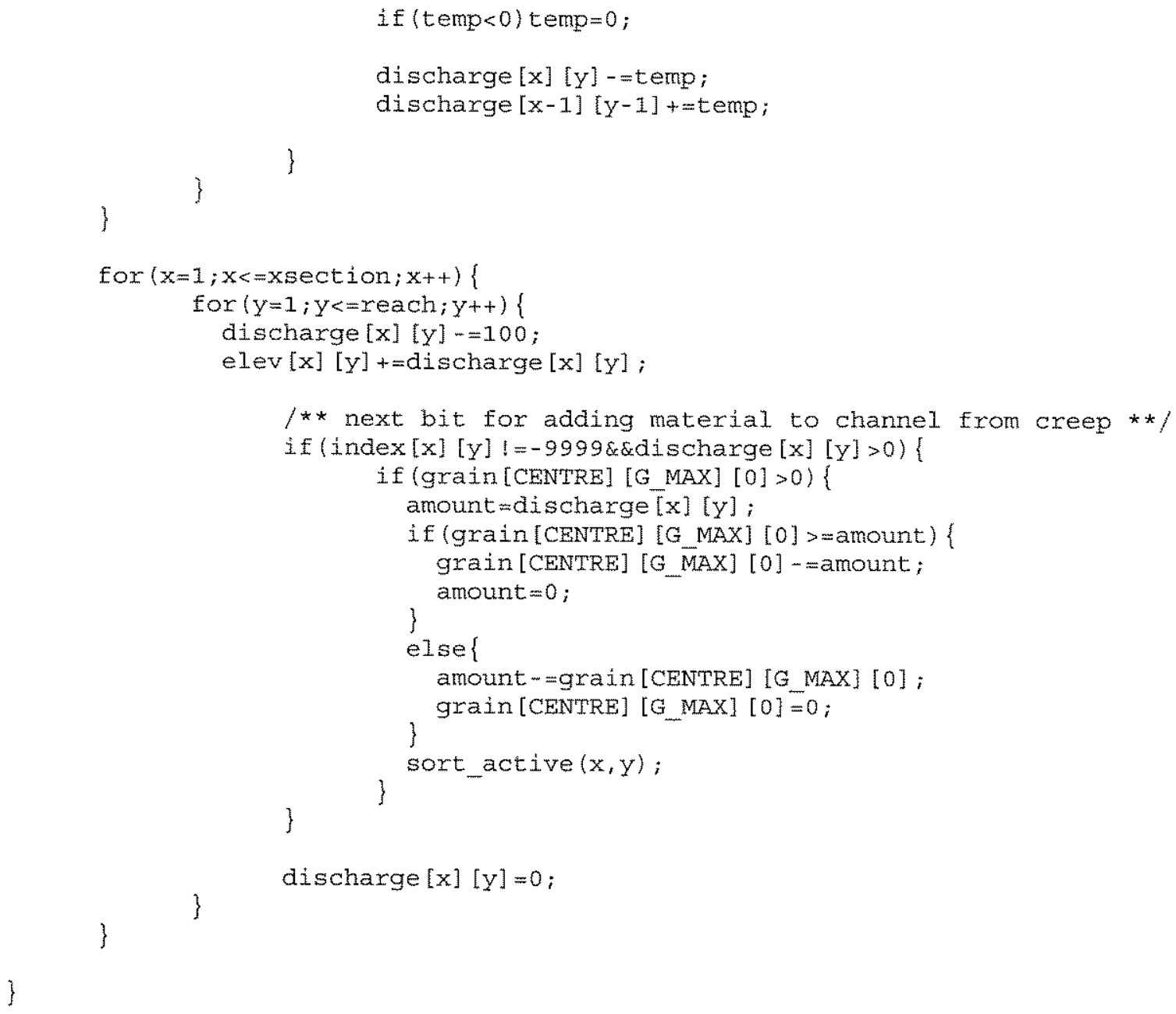




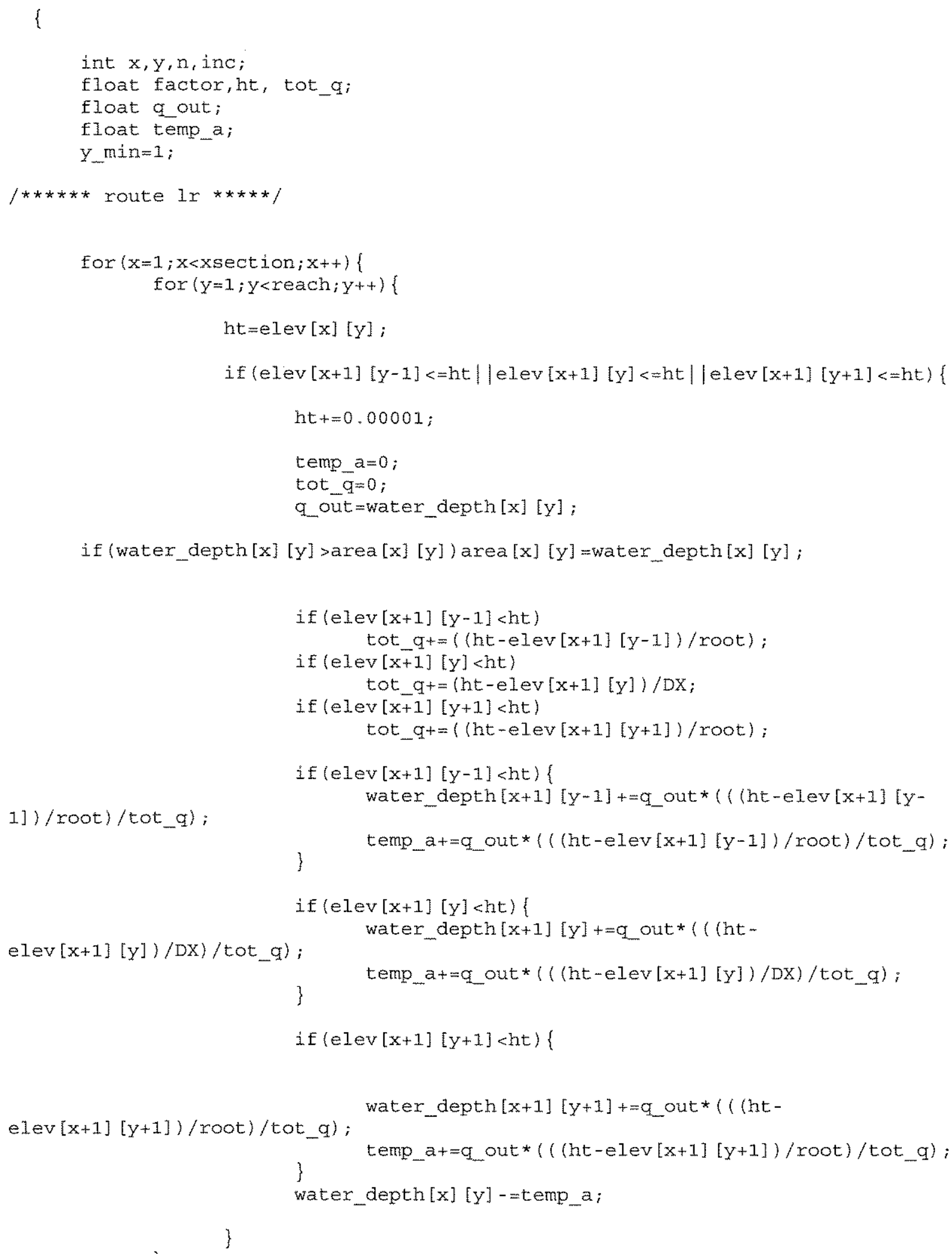




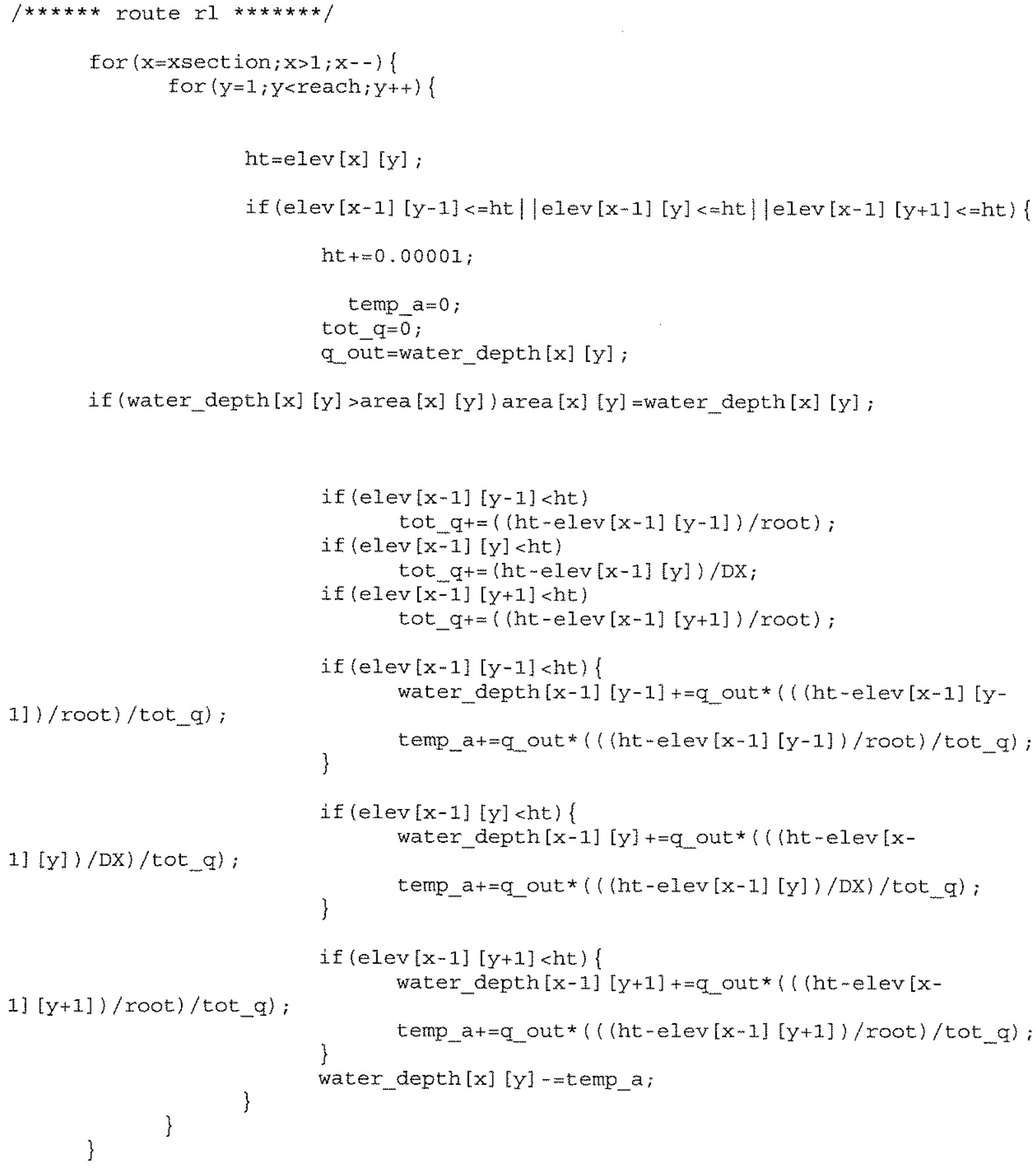




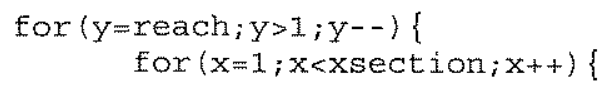




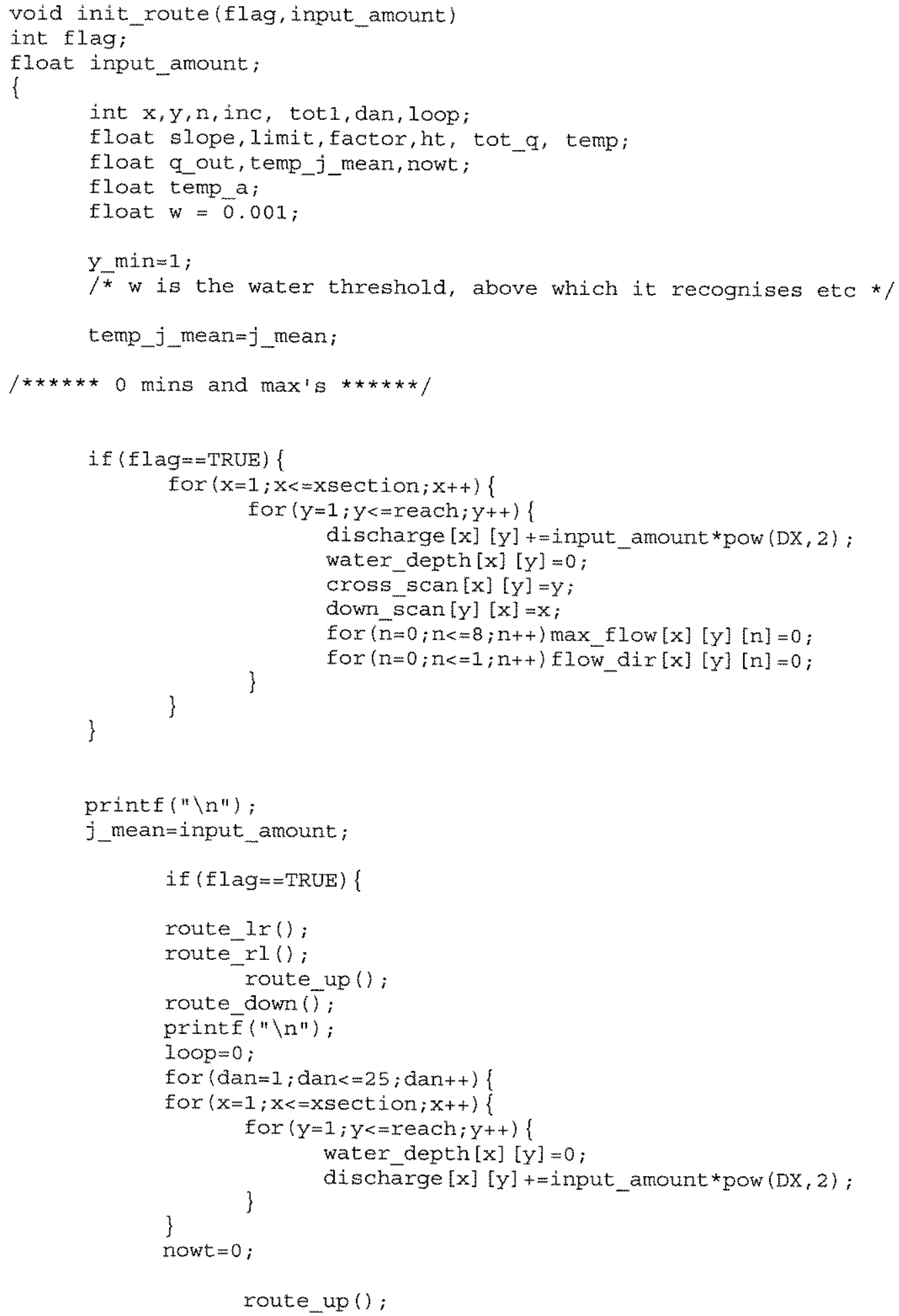




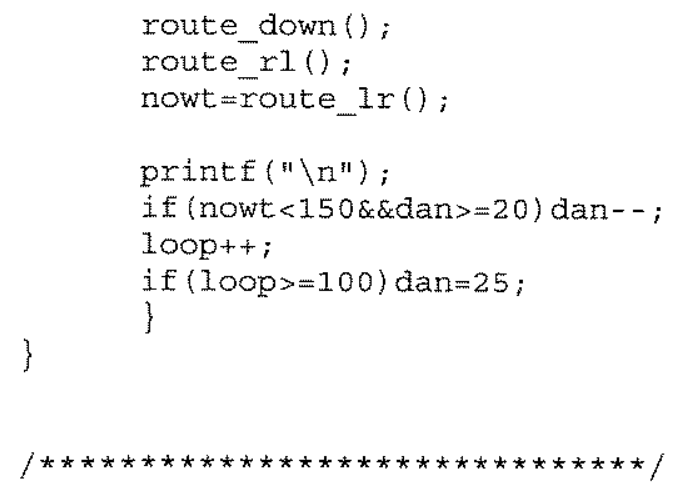

if (water_depth $[x][y]<w \& \& w a t e r$ depth $[x+1][y]<w \& \& w a t e r \operatorname{depth}[x+2][y]<w \& \& w a t e$ $r$ depth $[x-1][y]<w \& \& w a t e r$ depth $[x-2][y]<w$

3] $[y]<w \& \& w a t e r \_d e p t h[x][y-1]<w$

$$
\text { \&\&water_depth }[x+3][y]<w \& \& w a t e r \_d e p t h[x-
$$

\&\&water_depth $[x][y+1]<w \& \& w a t e r$ depth $[x][y-$

$2]<w \& \& w a t e r$ depth $[x][y+2]<w \& \& w a t e r$ depth $[x][y+3]<w$

\&\&water depth $[\mathrm{x}][\mathrm{y}-3]<w)\{$

\} water depth $[\mathrm{x}][y]=-9999$;

\}

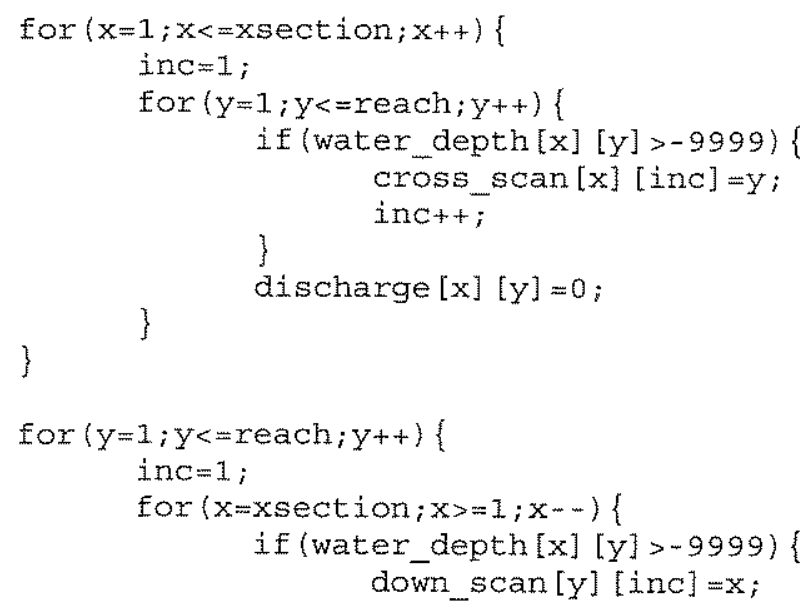




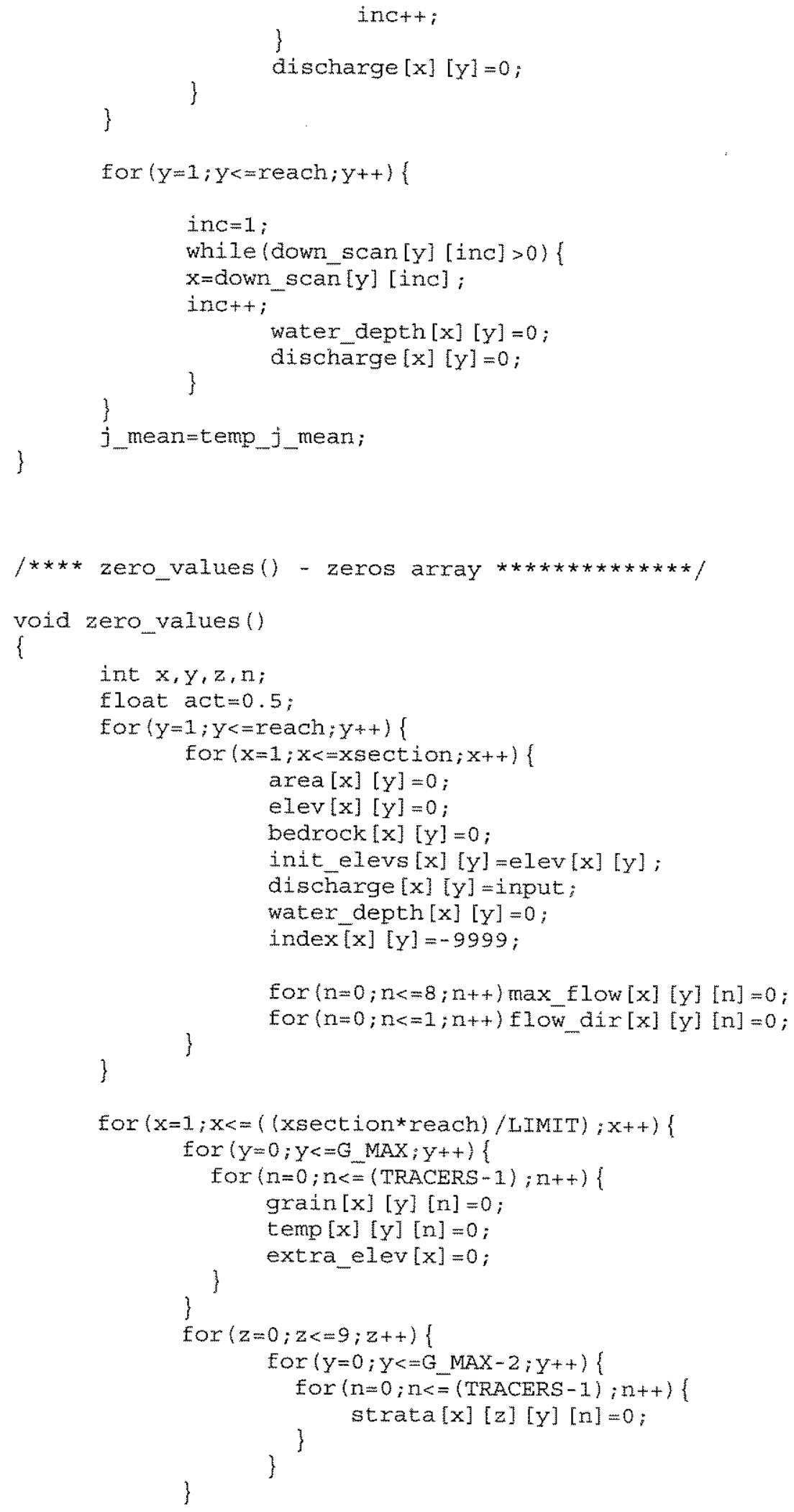




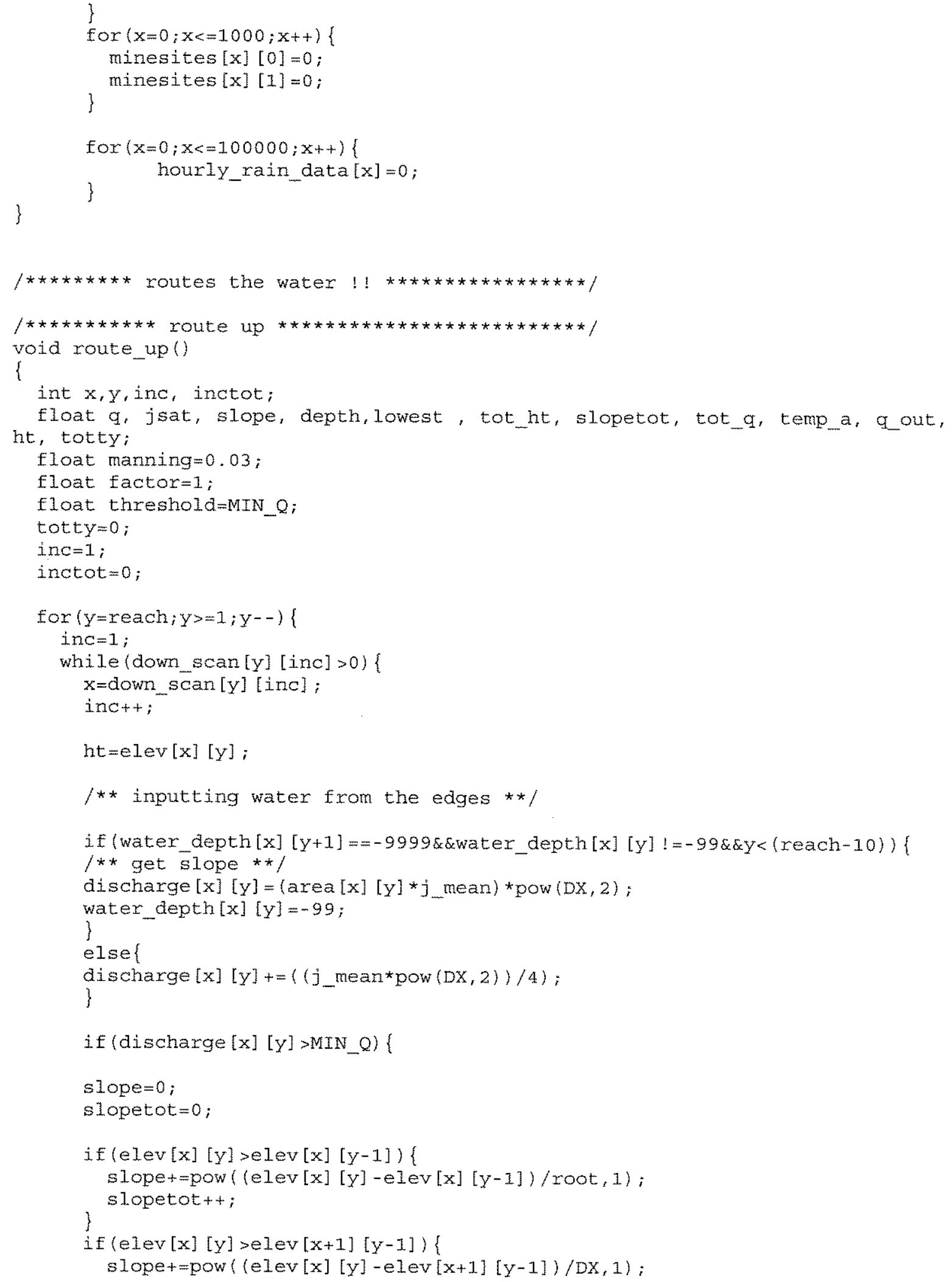




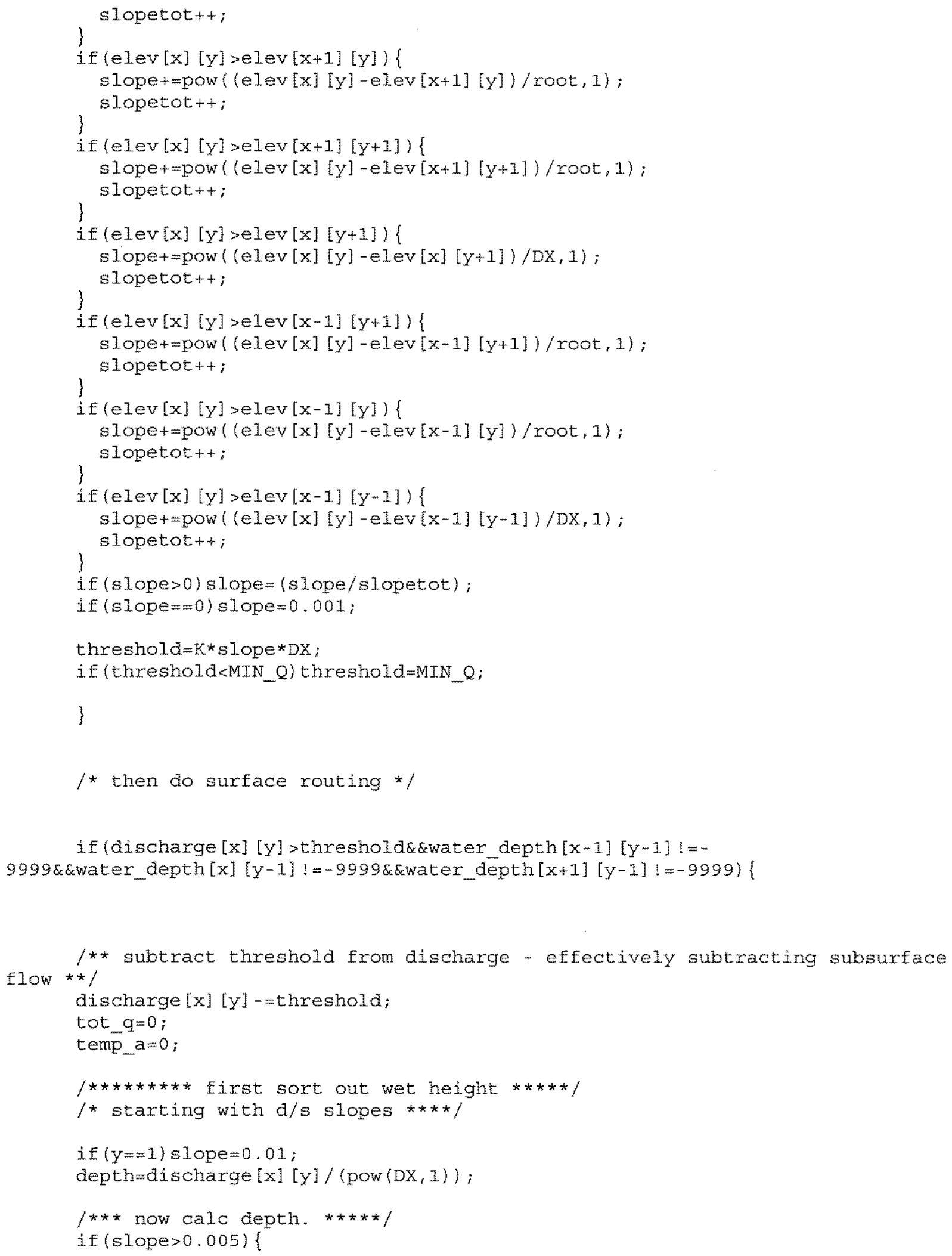




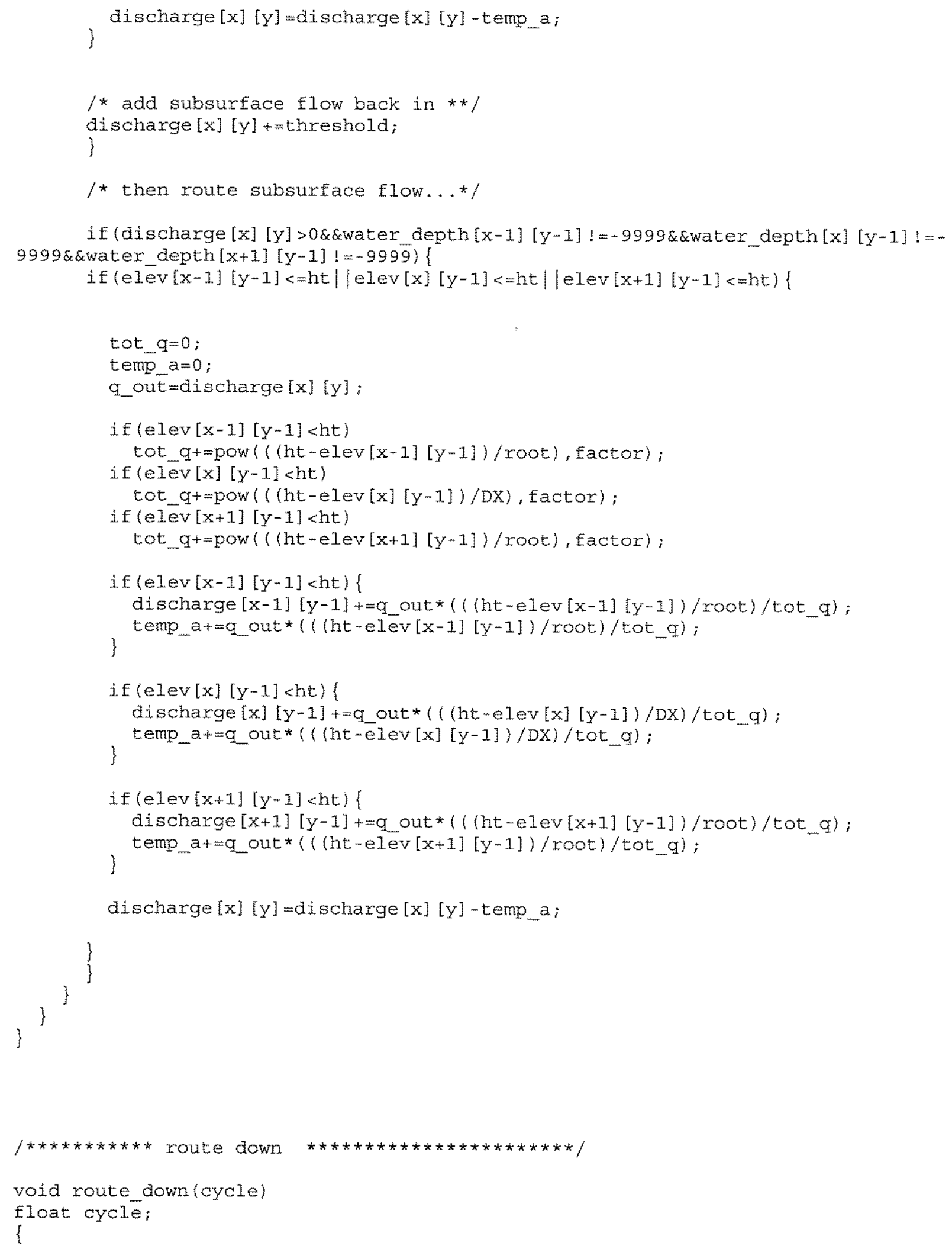


int $x, y$, inc, inctot;

float $q$, jsat, slope, depth, lowest, tot_ht, slopetot, tot_q, temp_a, q_out, ht, totty;

float manning $=0.03$;

float factor $=1$;

float threshold=MIN $Q$;

totty $=0$;

inc $=1$;

inctot $=0$;

for $(y=1 ; y<=r e a c h ; y++)\{$

inctot $+=$ inc:

inc $=1$;

while (down scan $[y][$ inc] $>0)$ ( $\mathrm{x}=$ down_scan $[y][\mathrm{inc}]$;

inct+;

$h t=\operatorname{lev}[\mathrm{x}][\mathrm{y}]$;

/* route water if not enough to flow */

if (water_depth $[\mathrm{x}][\mathrm{y}-1]=-9999 \& \&$ water_depth $[\mathrm{x}][\mathrm{y}] !=-99)\{$

discharge $[x][y]=(\operatorname{area}[x][y] * j$ mean $) *$ pow $(D x, 2)$;

water_depth $[x][y]=-99$;

\}

else

discharge $[x][y]+=((j$ mean*pow $(D X, 2)) / 4)$;

\}

if (discharge $[\mathrm{x}][\mathrm{y}]>\operatorname{MIN} \_$) (

slope $=0$;

slopetot $=0$

if (elev $[x][y]>\operatorname{lev}[x][y-1])($ slopet=pow ( (elev $[x][y]-$ elev $[x][y-1]) / \operatorname{root}, 1)$; ) slopetot $++;$

if $(e \operatorname{lev}[x][y]>\operatorname{lev}[x+1][y-1])\{$

slope $+=$ pow $($ (elev $[x][y]-\operatorname{elev}[x+1][y-1]) / D X, 1)$;

\}

slopetot+ti

if (elev $[x][y]>\operatorname{elev}[x+1][y])($

slopet=pow $((e l e v[x][y]-e l e v[x+1][y]) /$ root, 1$)$;

)

slopetot + ;

if (elev $[x][y]>\operatorname{elev}[x+1][y+1])\{$

slope $+=$ pow $((\operatorname{elev}[x][y]-\operatorname{lev}[x+1][y+1]) / \operatorname{root}, 1)$;

\}

slopetot+t;

if $(e \operatorname{lev}[x][y]>\operatorname{lev}[x][y+1])\{$ 


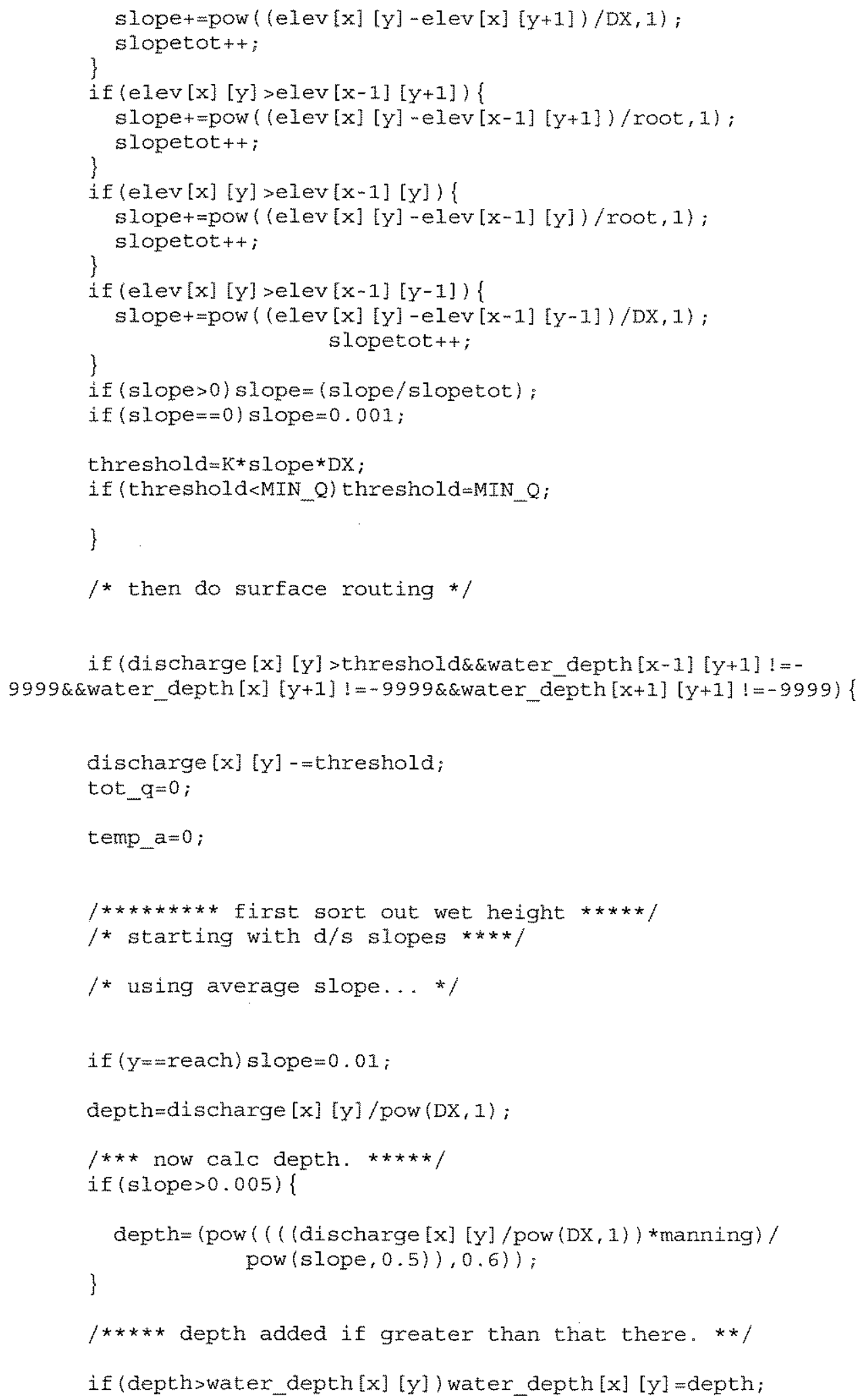




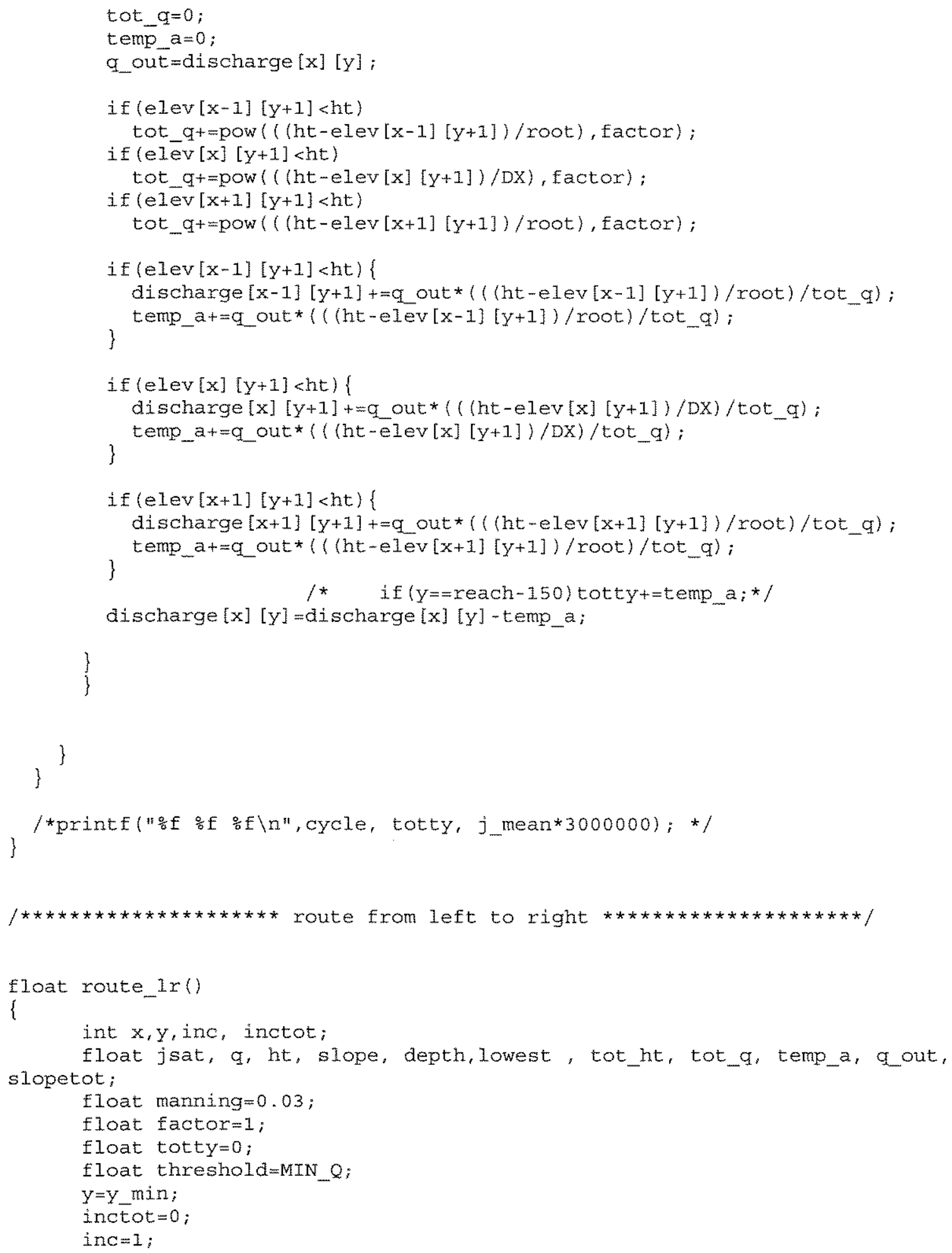




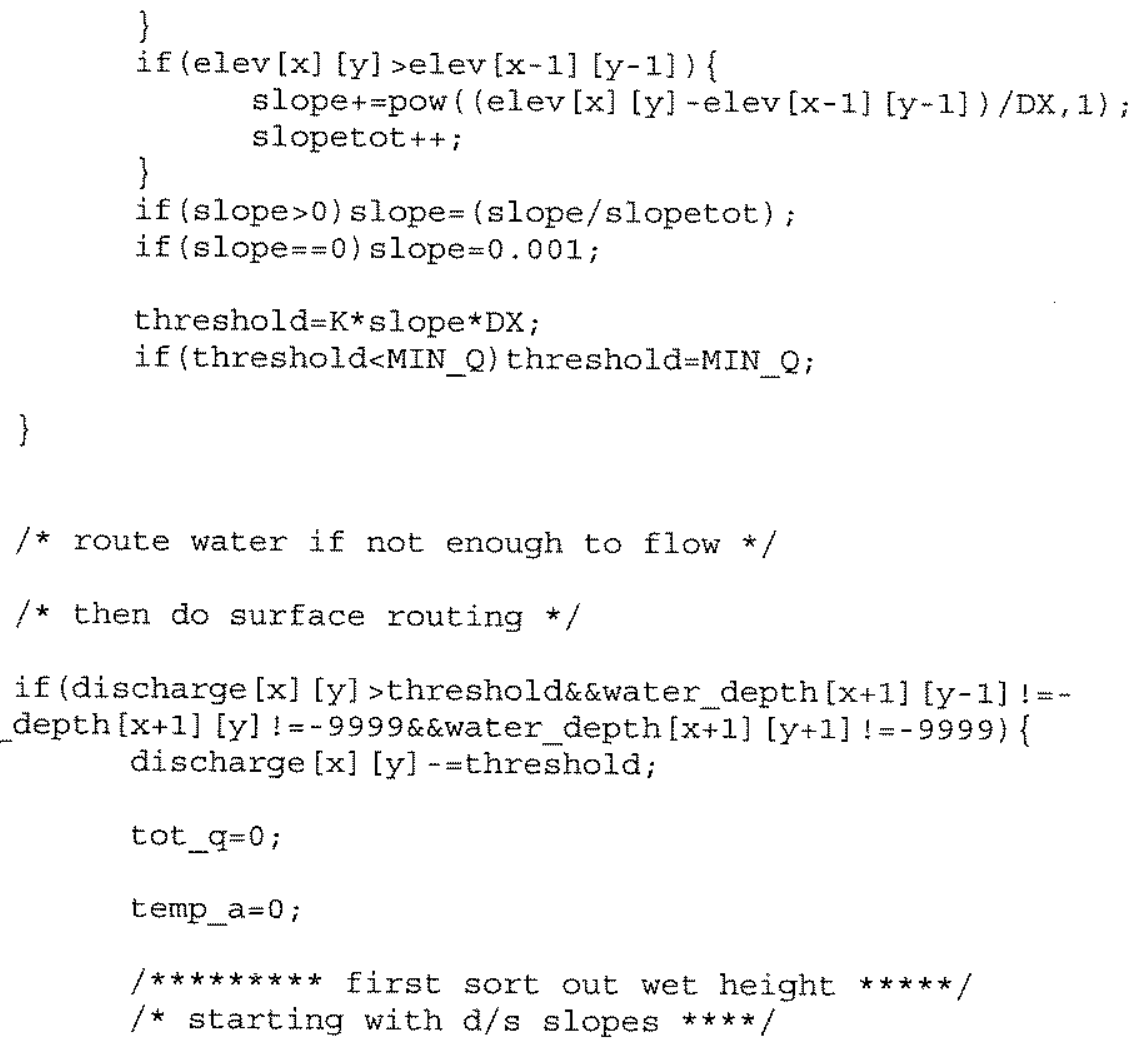




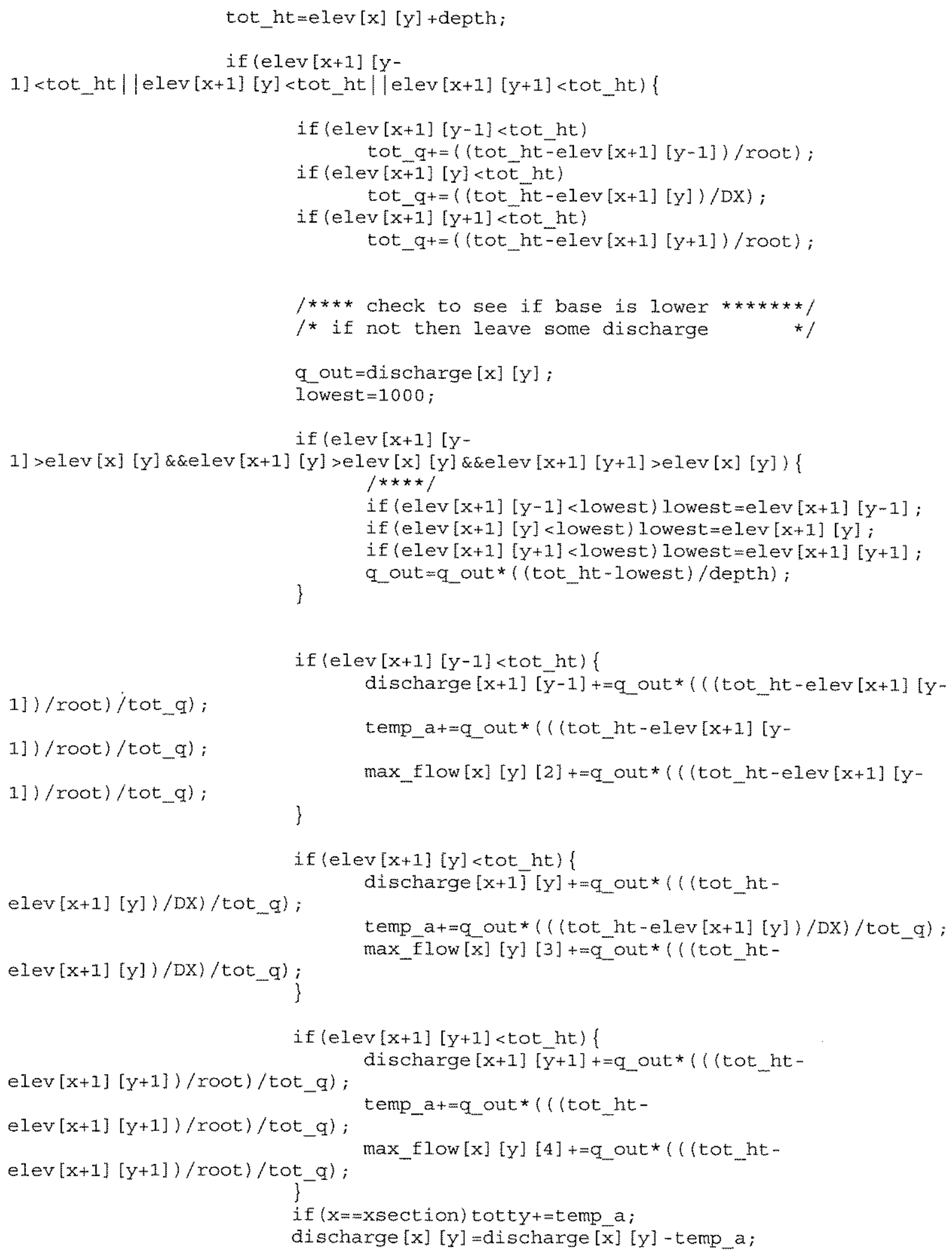




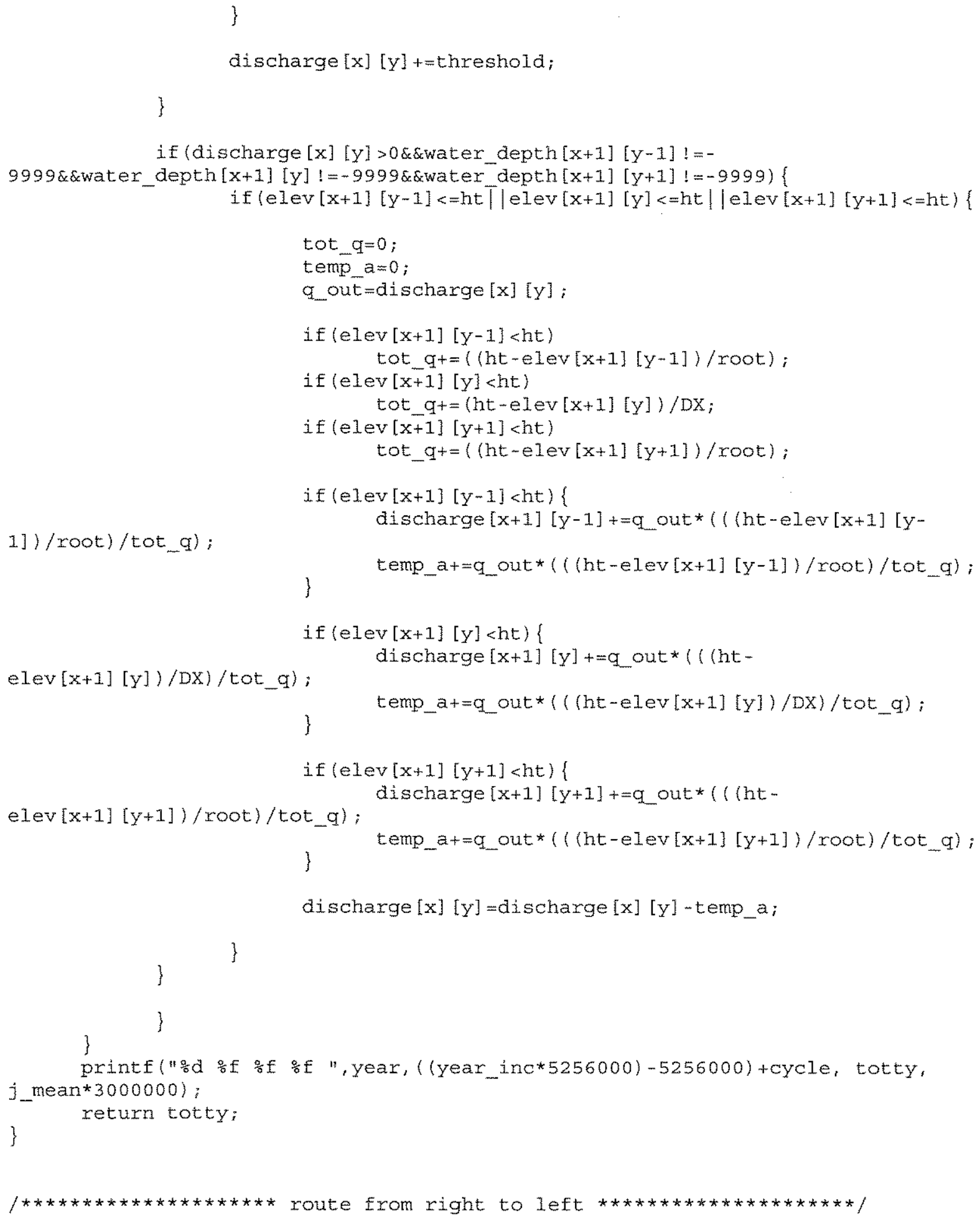




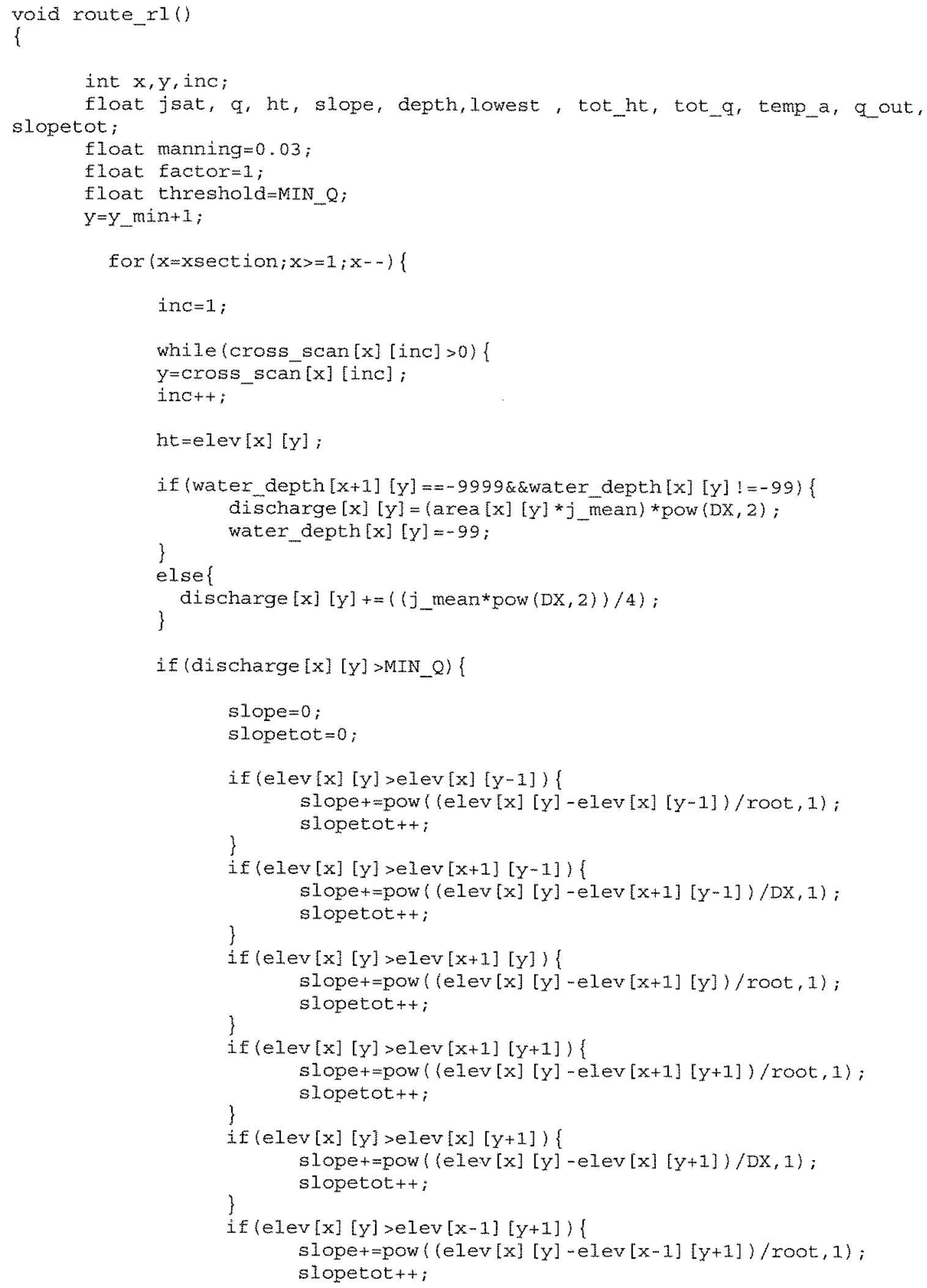




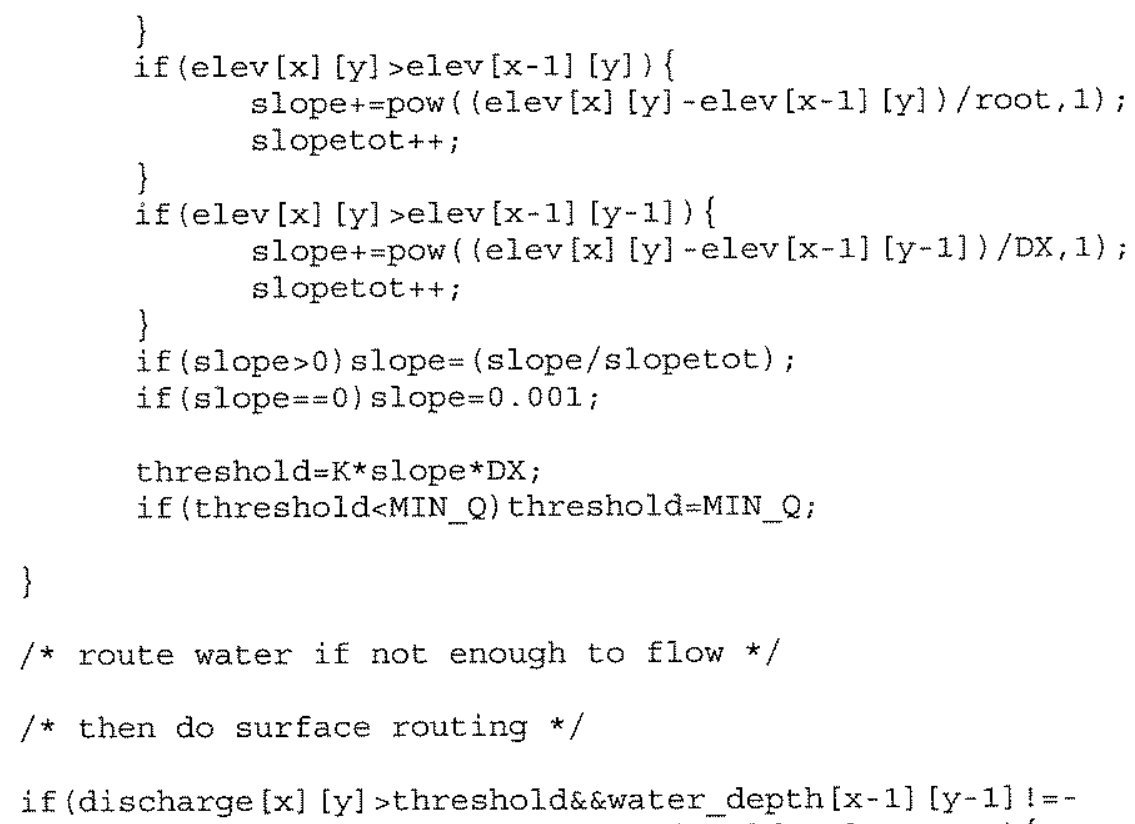

$$
\text { if }(e \operatorname{ev}[x-1][y-1]<\text { tot } h t)
$$




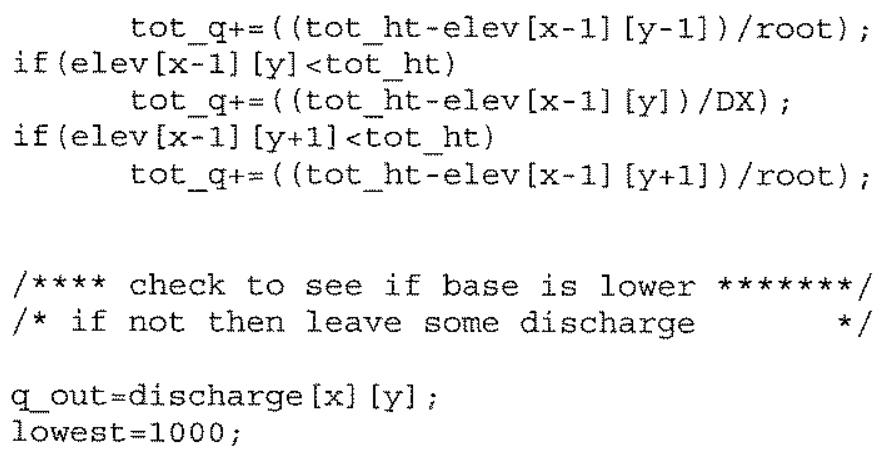

1] $[y+1] / / \operatorname{root}) / \operatorname{tot} q$ ) ;

1] $[y+1] / /$ root $/ /$ tot_q):

1] $[y+1] / / \operatorname{root}) /$ tot $q$ q) ; temp_a+=q_out*(1) tot_ht-elev[x- 
if $(e \operatorname{lev}[x-1][y-1]<=h t||$ elev $[x-1][y]<=h t||$ elev $[x-1][y+1]<=h t)($

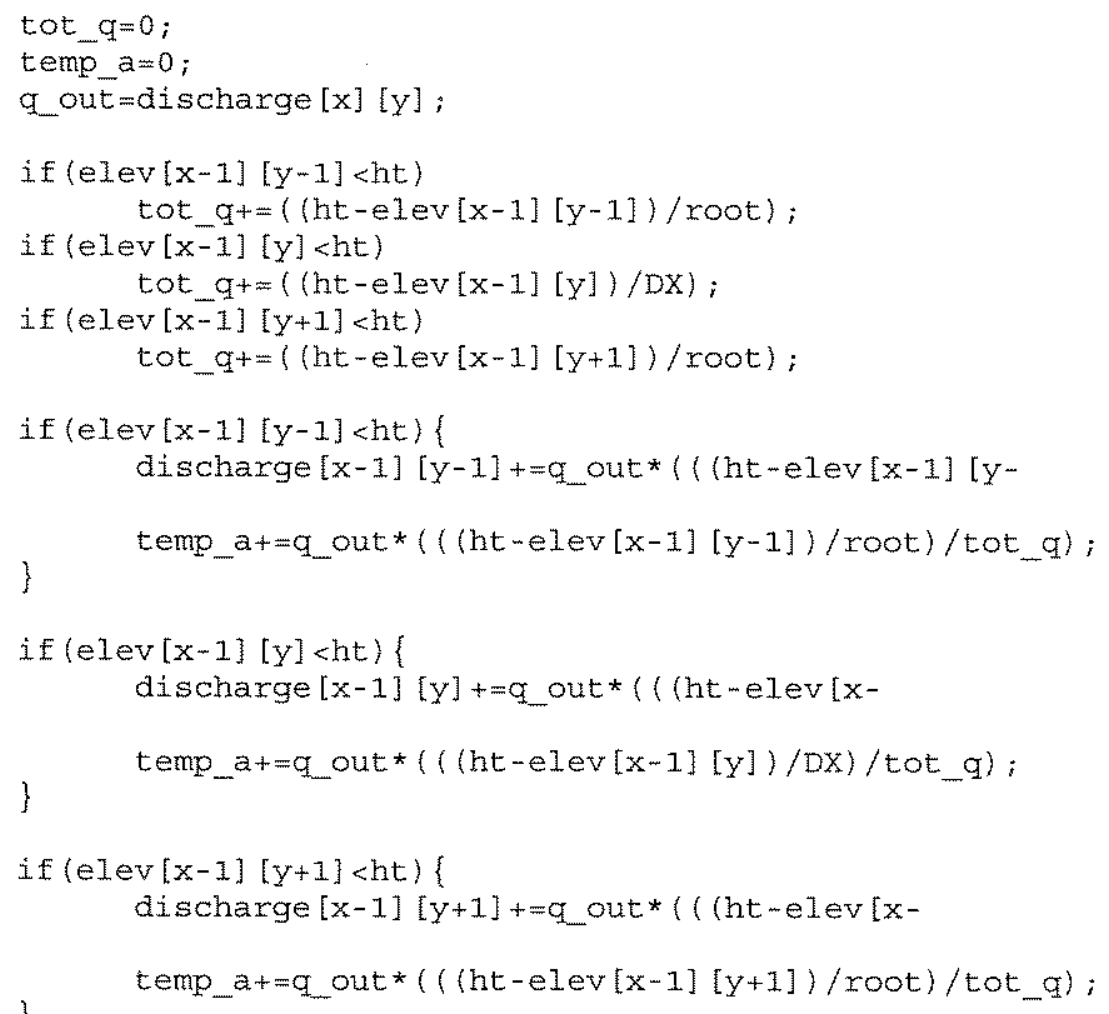
discharge $[x-1][y-1]+=q$ out* $(($ ht -elev $[x-1][y-$

\section{)} temp_a+=q_out* $(((h t-e l e v[x-1][y-1]) /$ root $) /$ tot_q $)$;

1])/root)/tot_q);

1] [y] $/ / \mathrm{DX}) /$ tot_q);

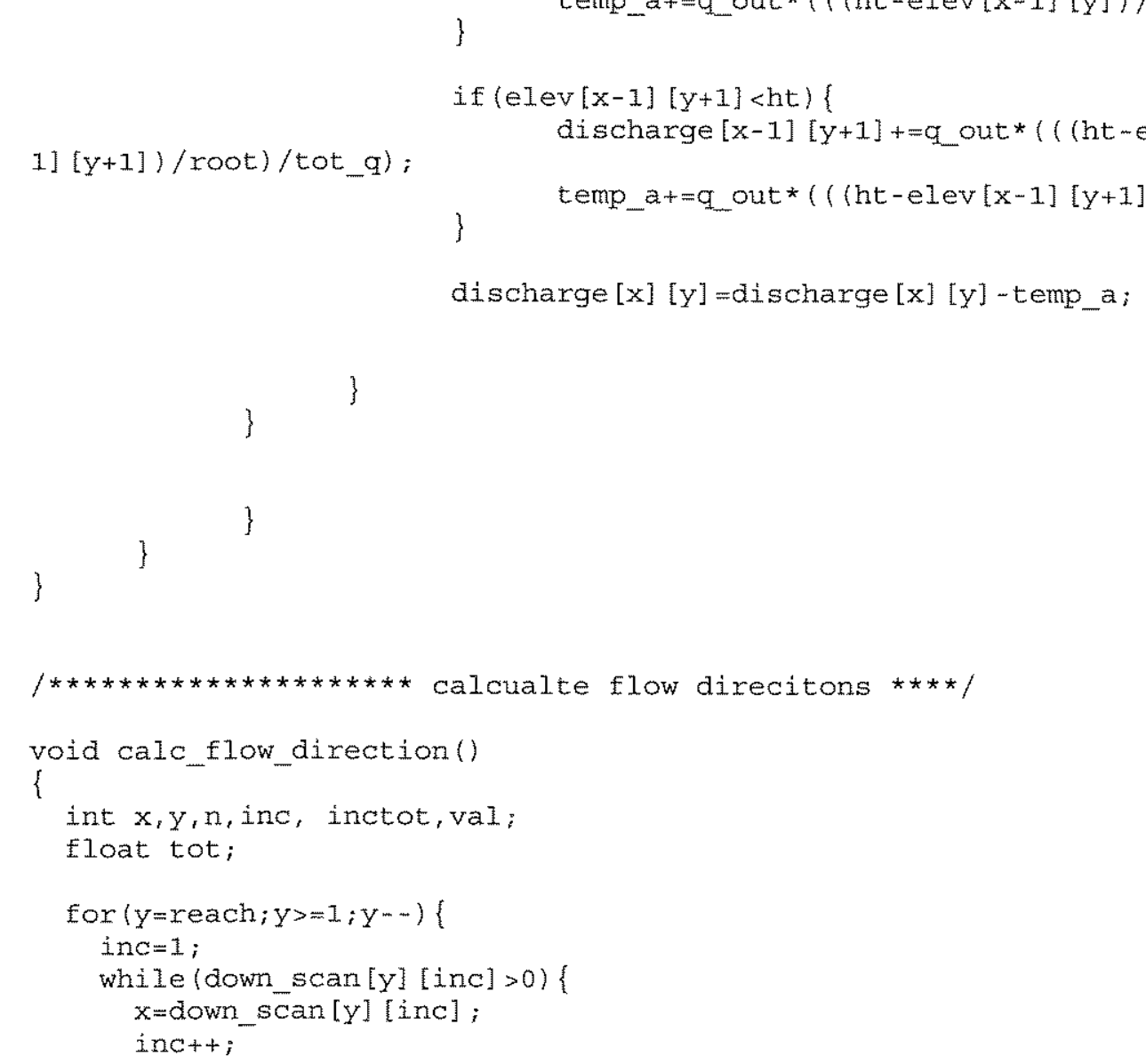




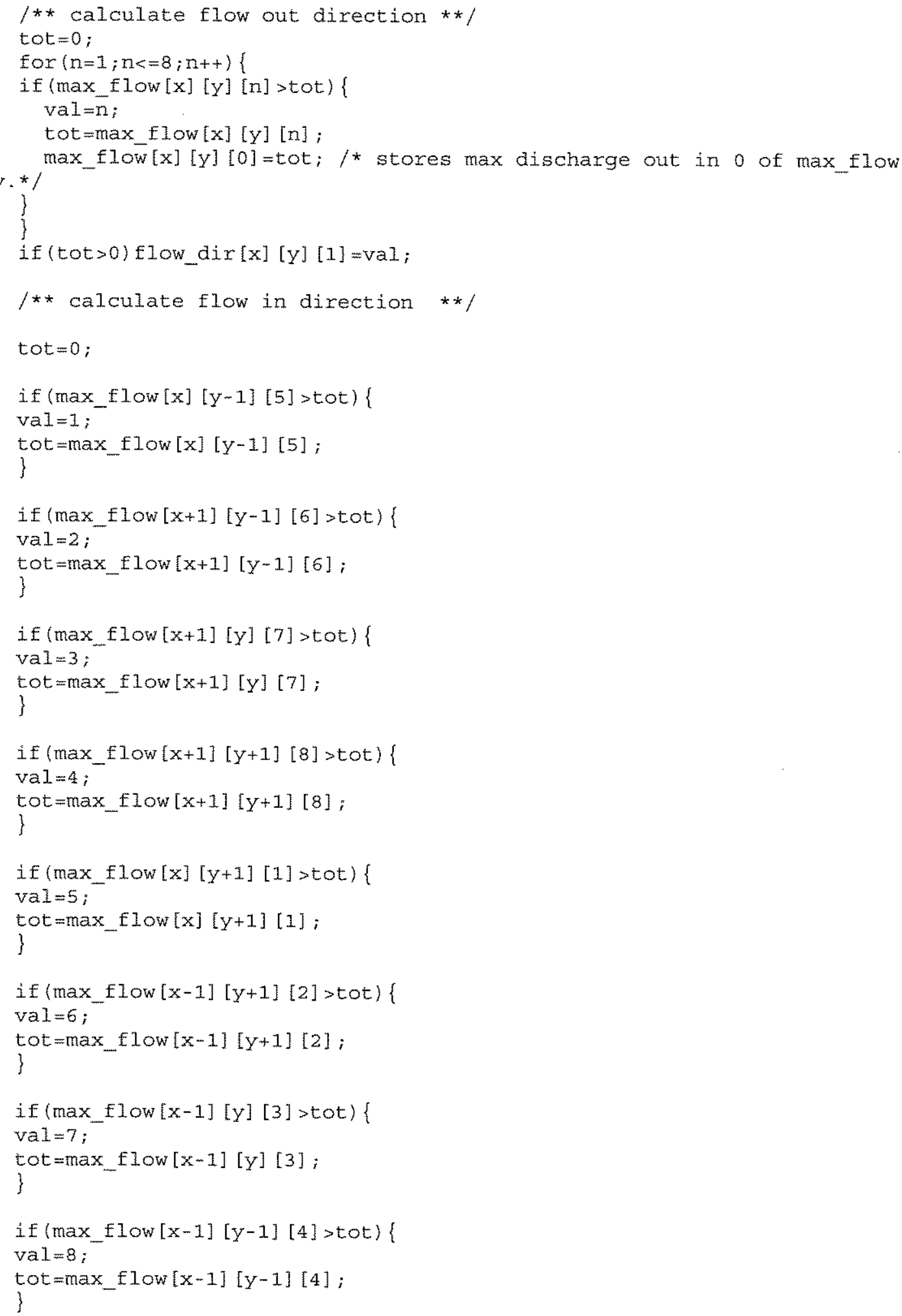




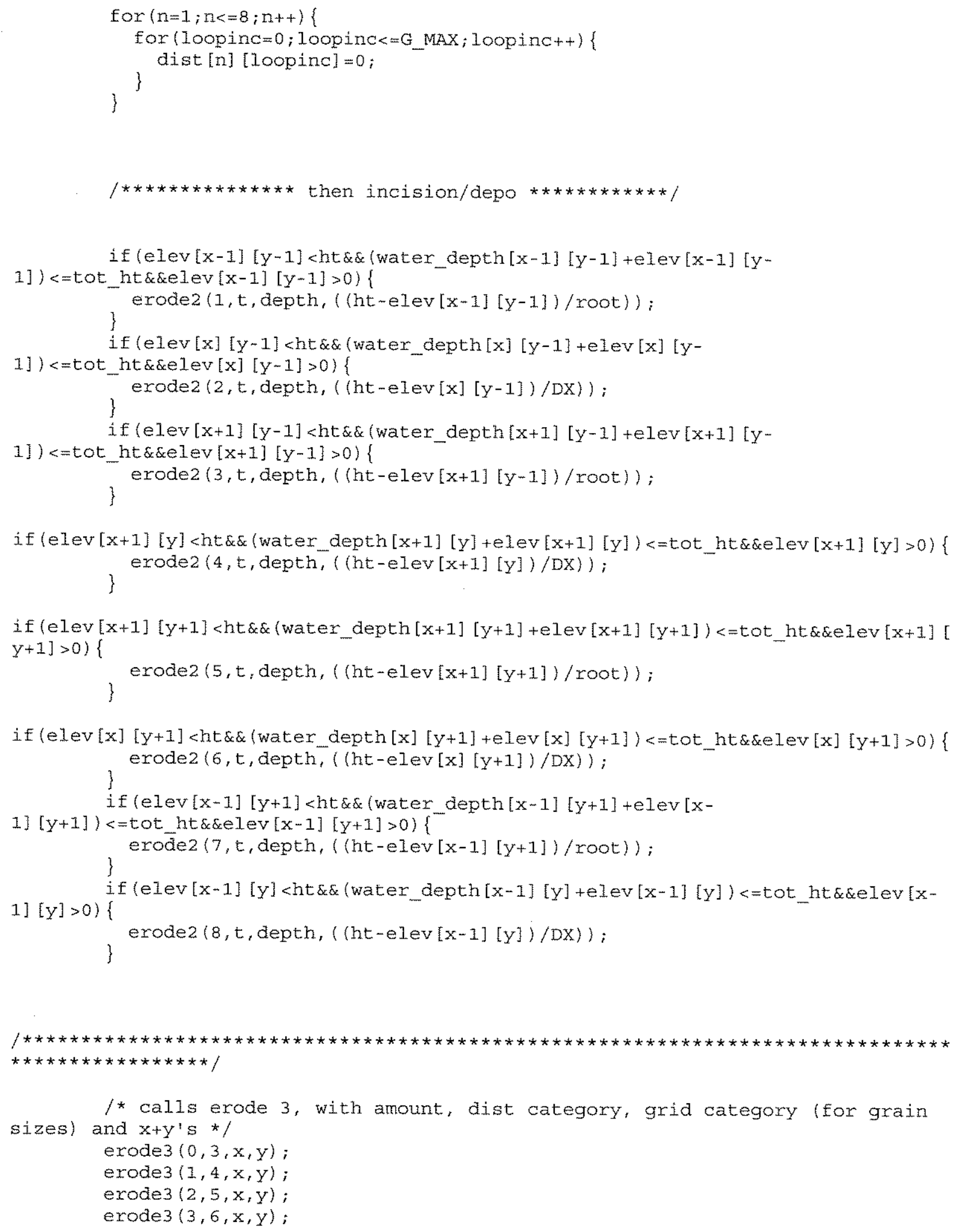




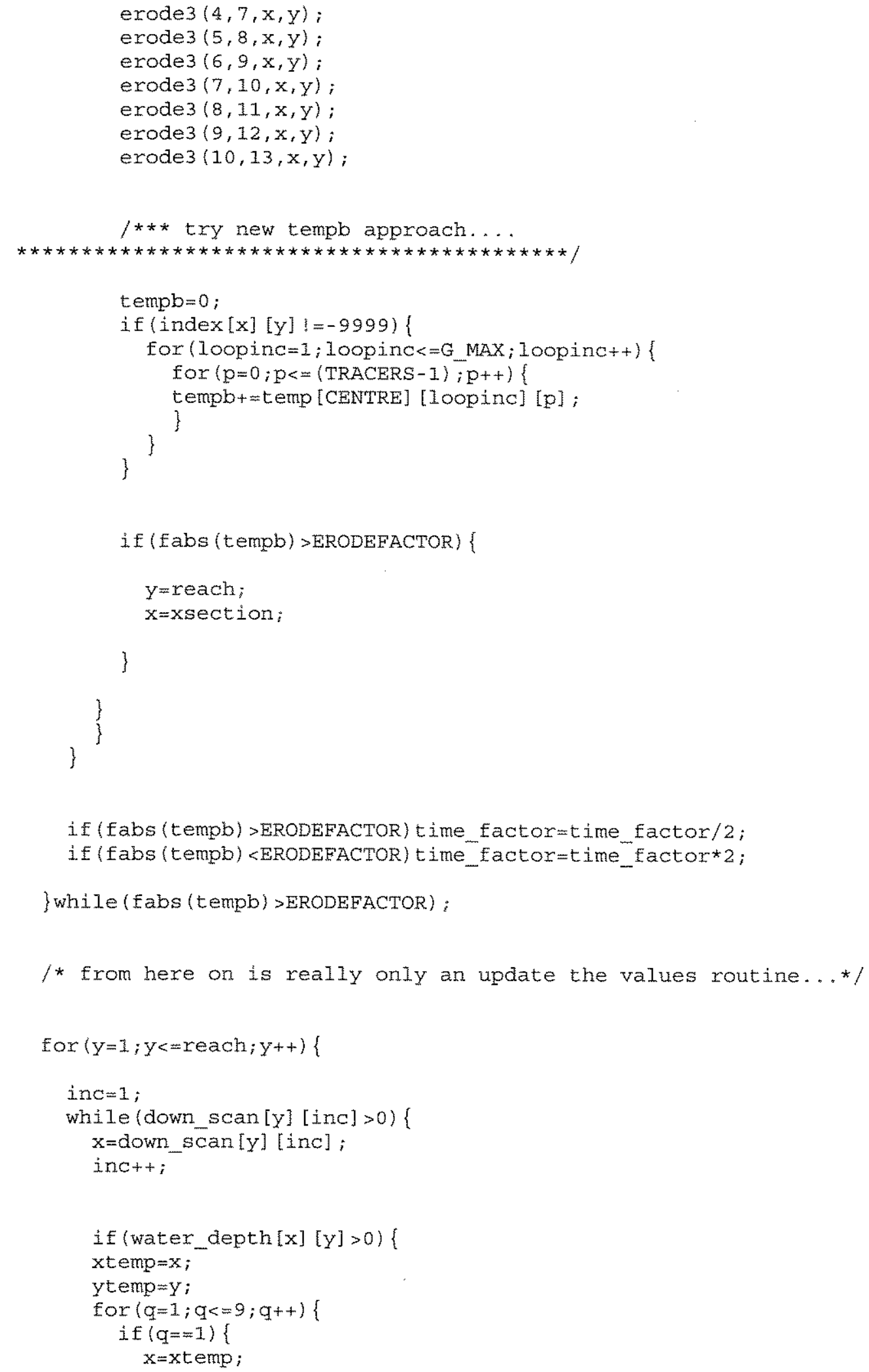




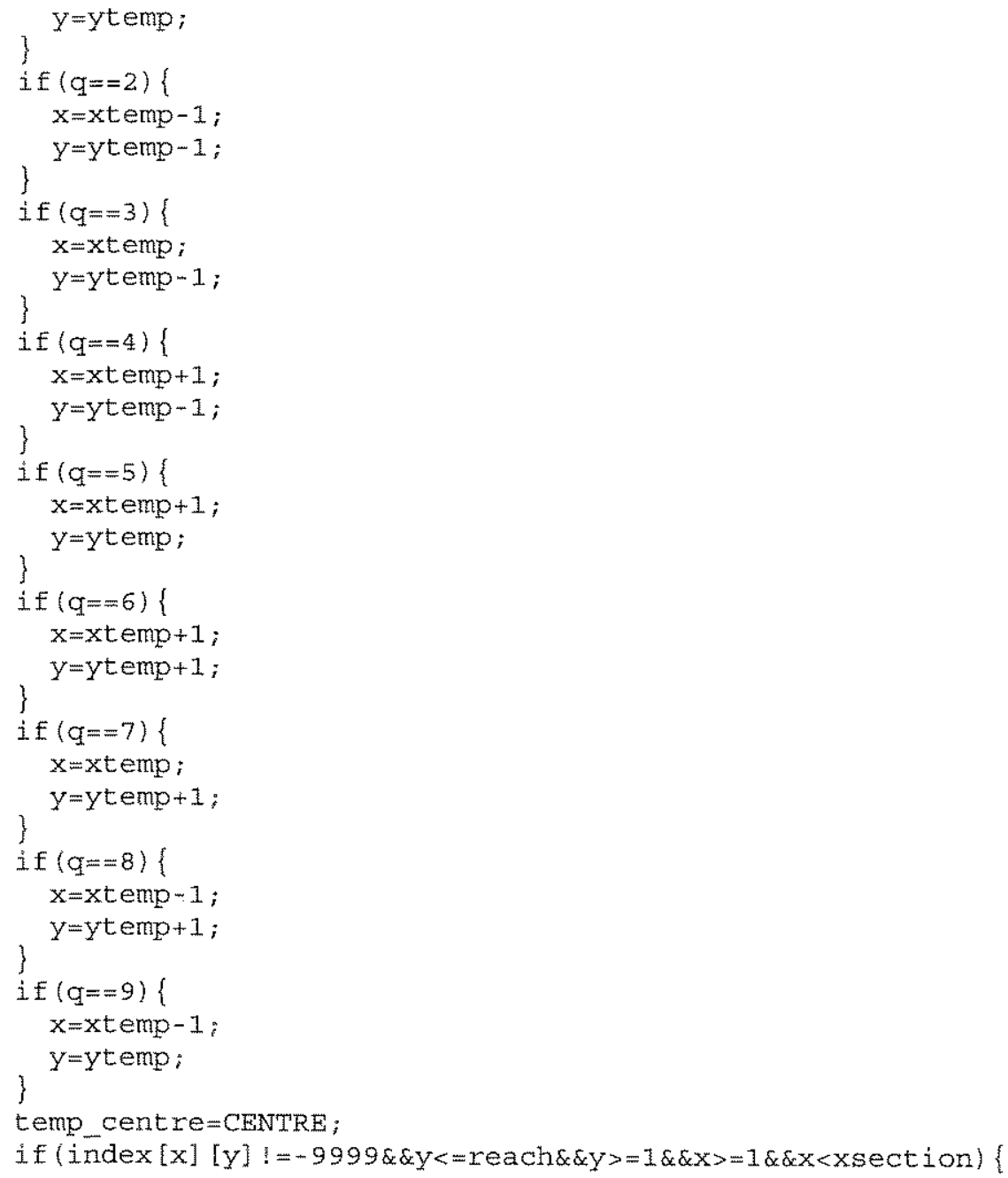




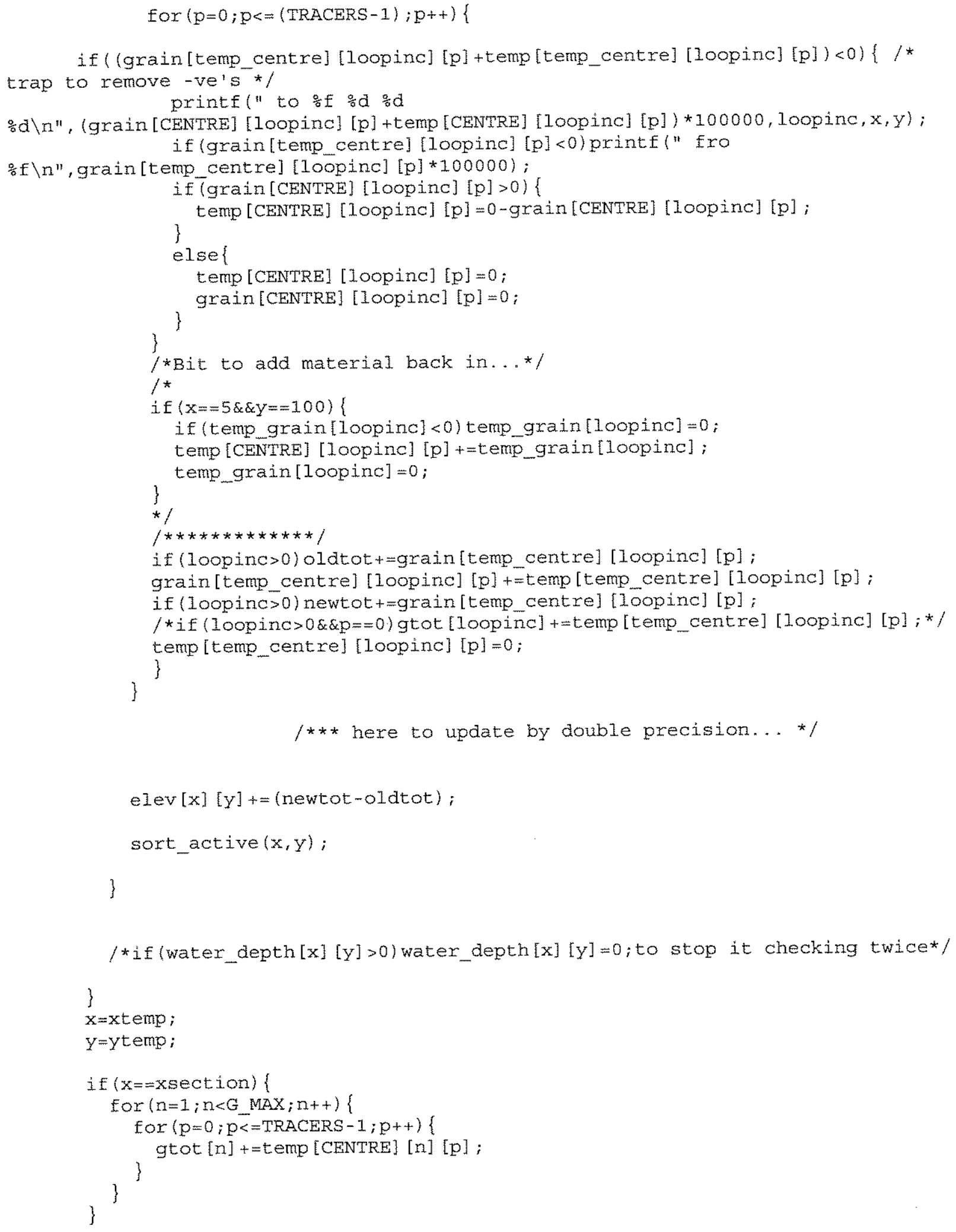




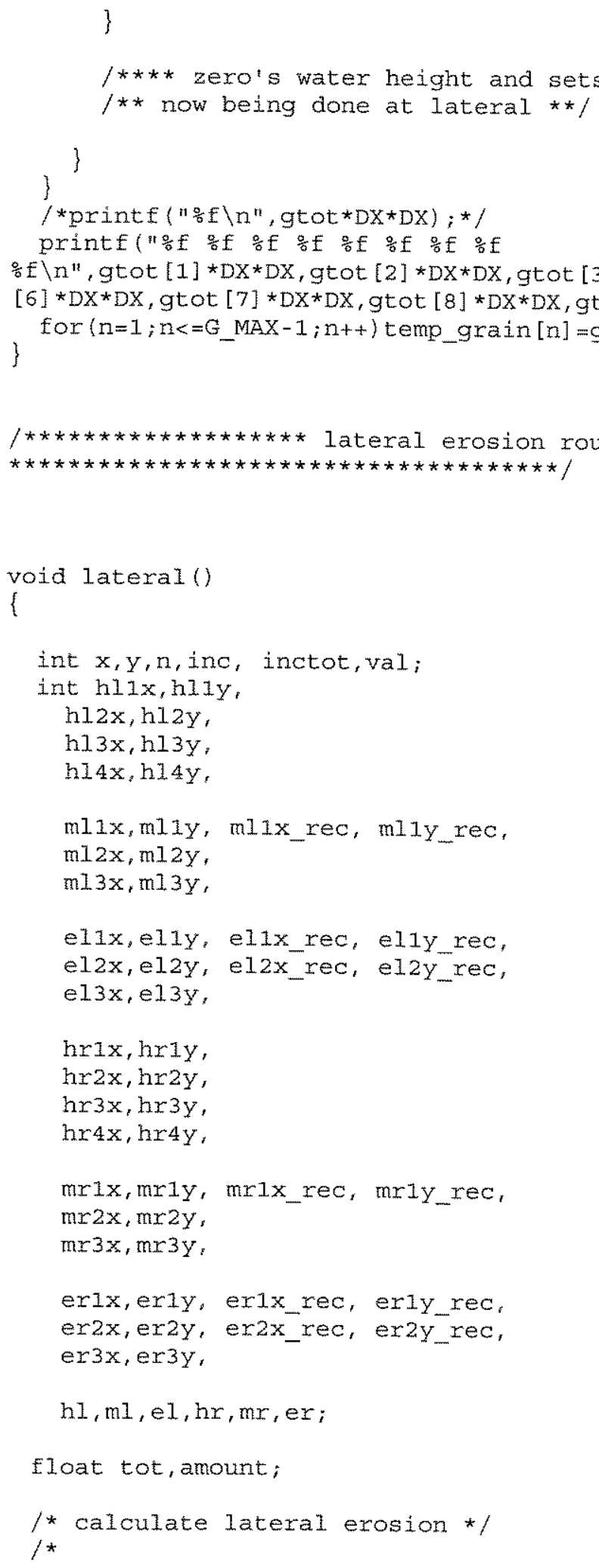




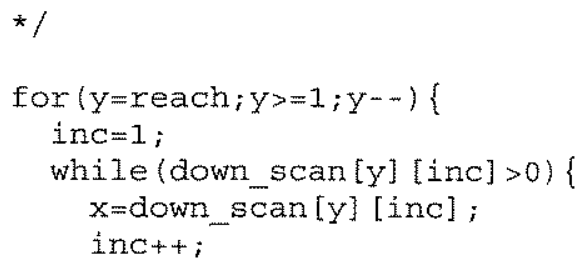

if (water_depth $[x][y]>0.0001 \& \&$ flow_dir $[x][y][0] !=0 \& \& f$ Iow dir $[x][y][1] !=0 \& \&(f 10 w d$

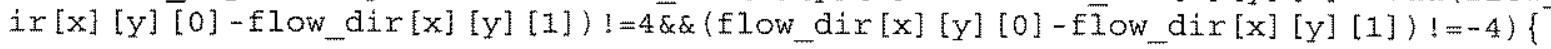

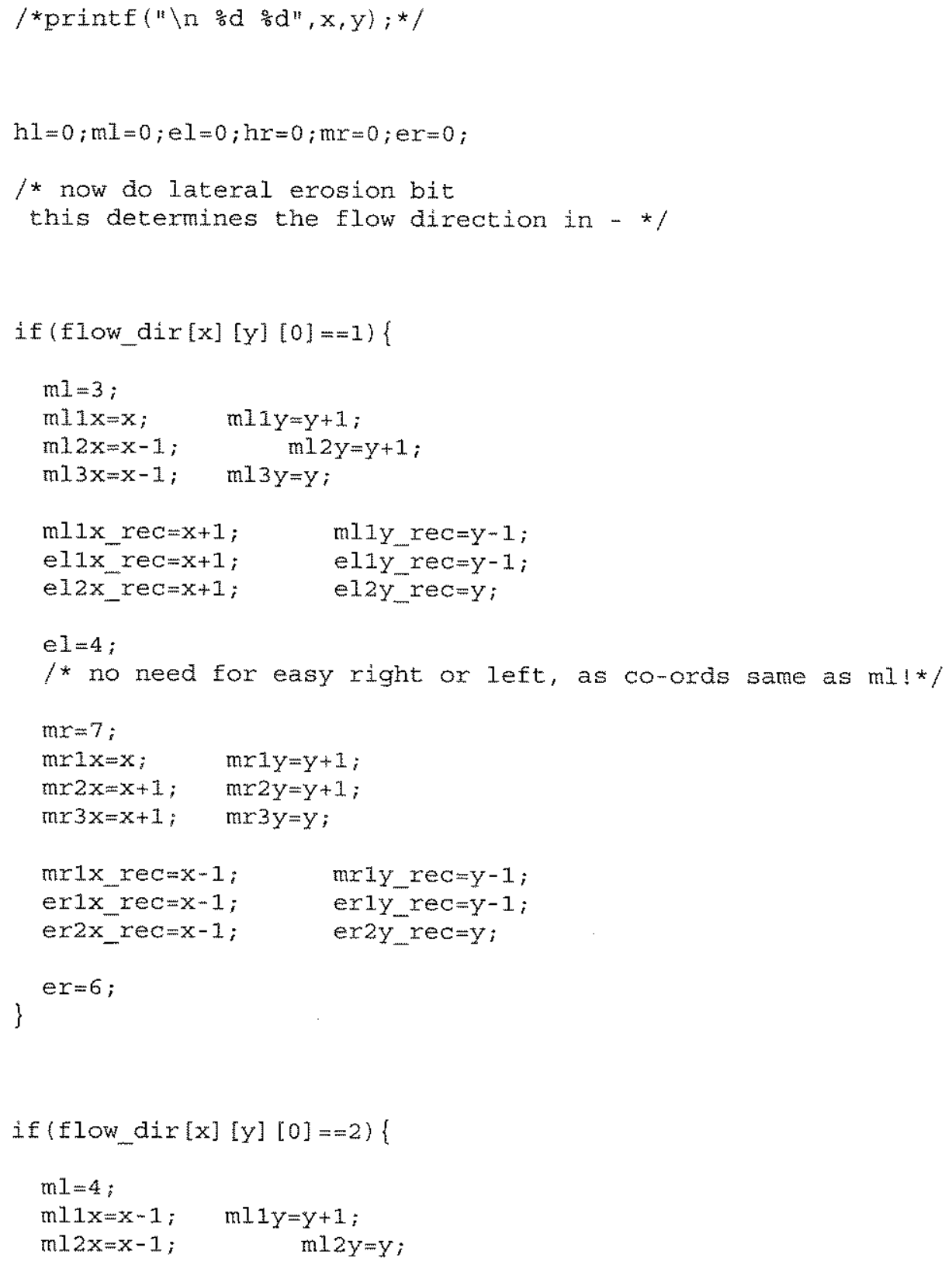




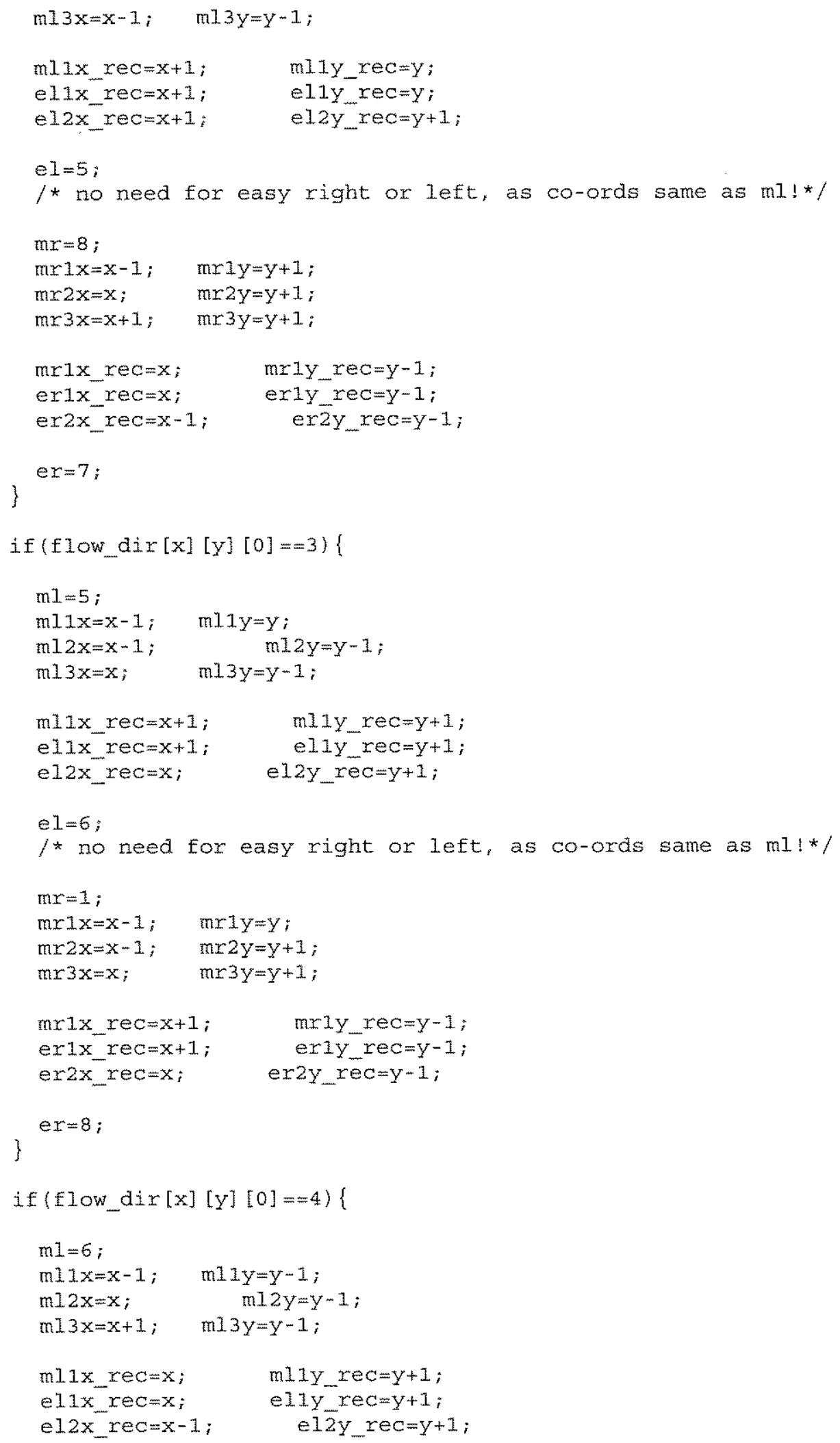




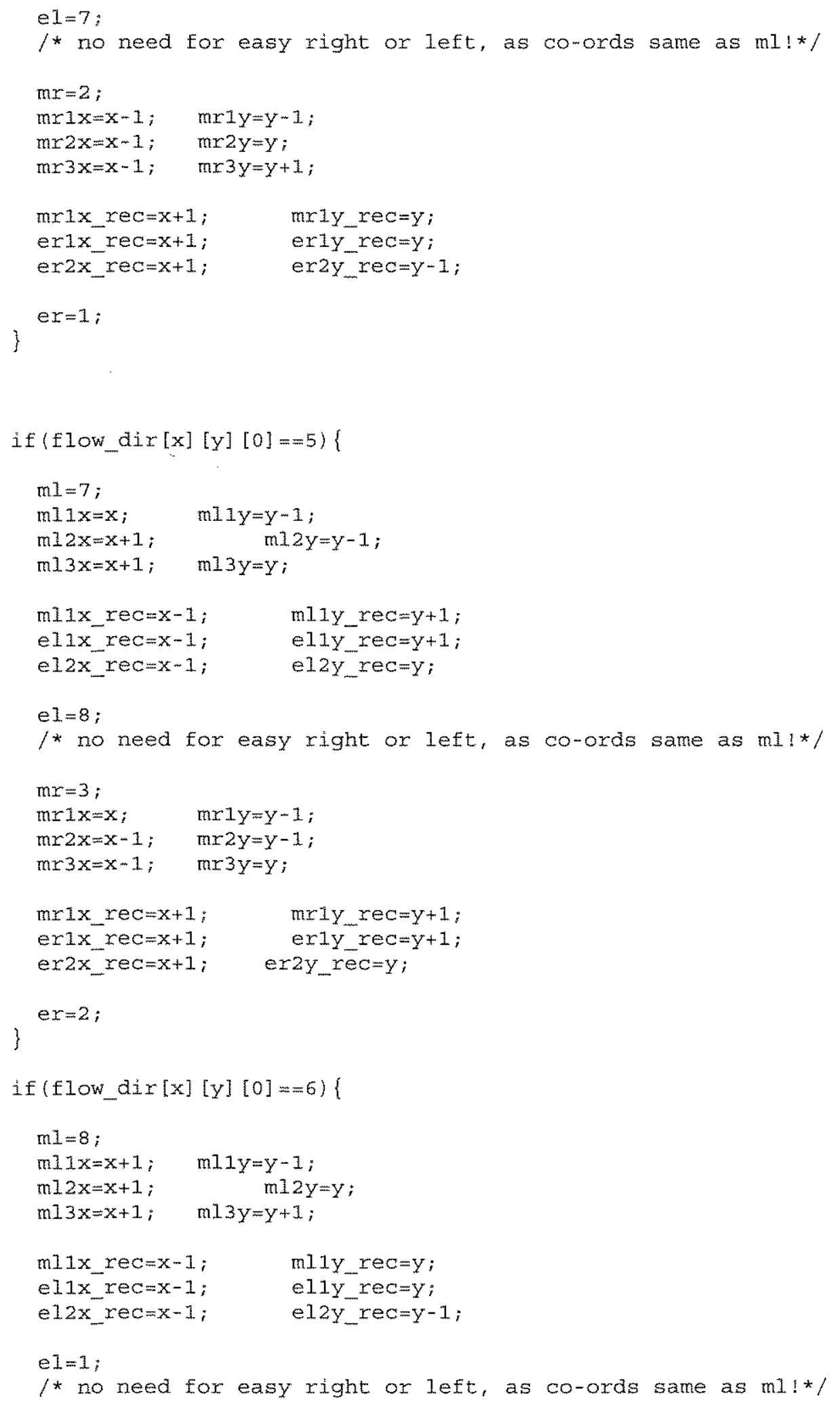




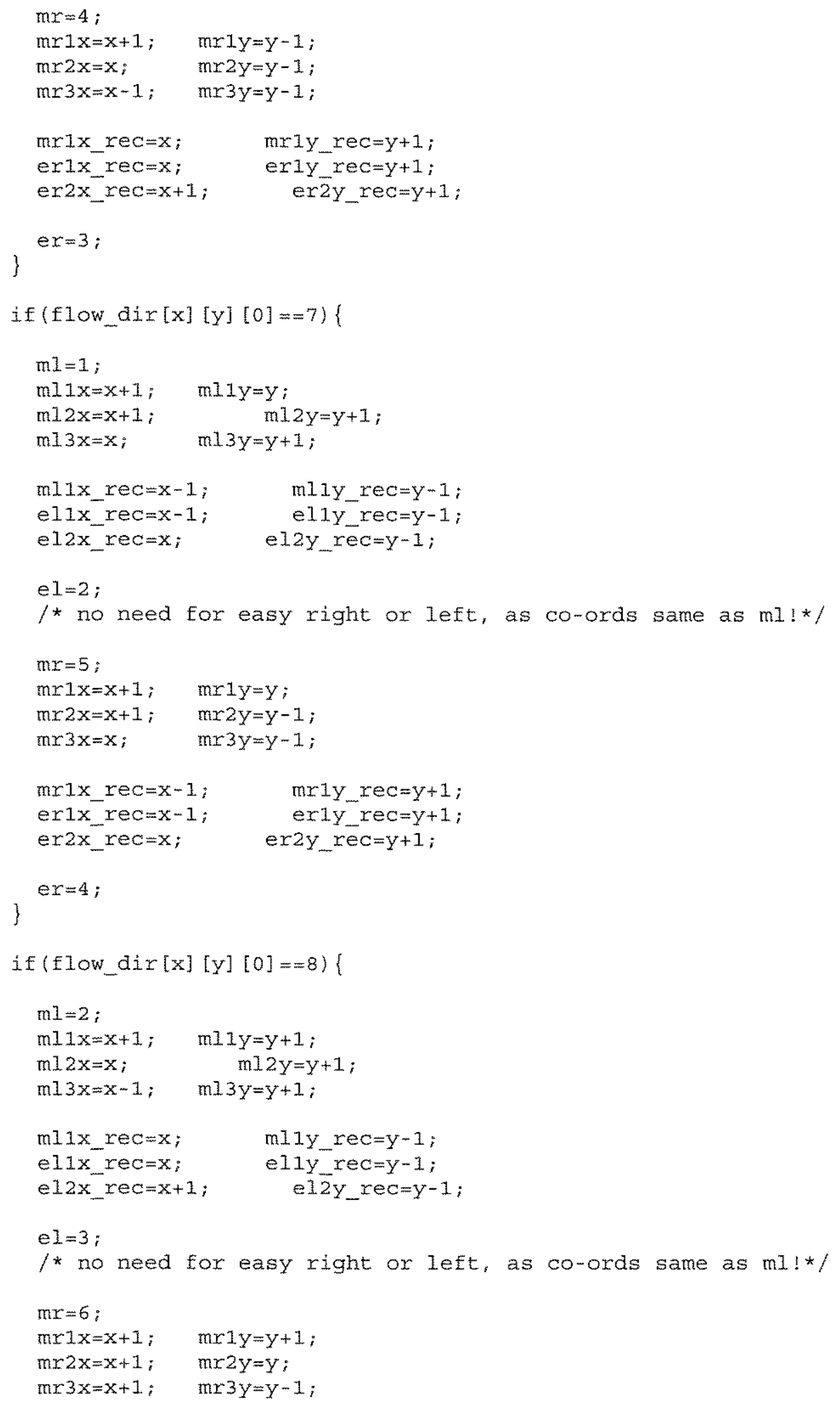




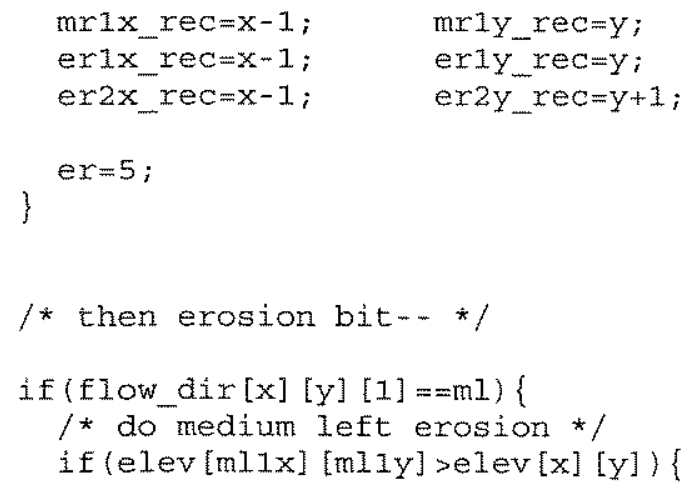

if $(e l e v[m 13 x][m 13 y]>\operatorname{elev}[x][y])\{$ 


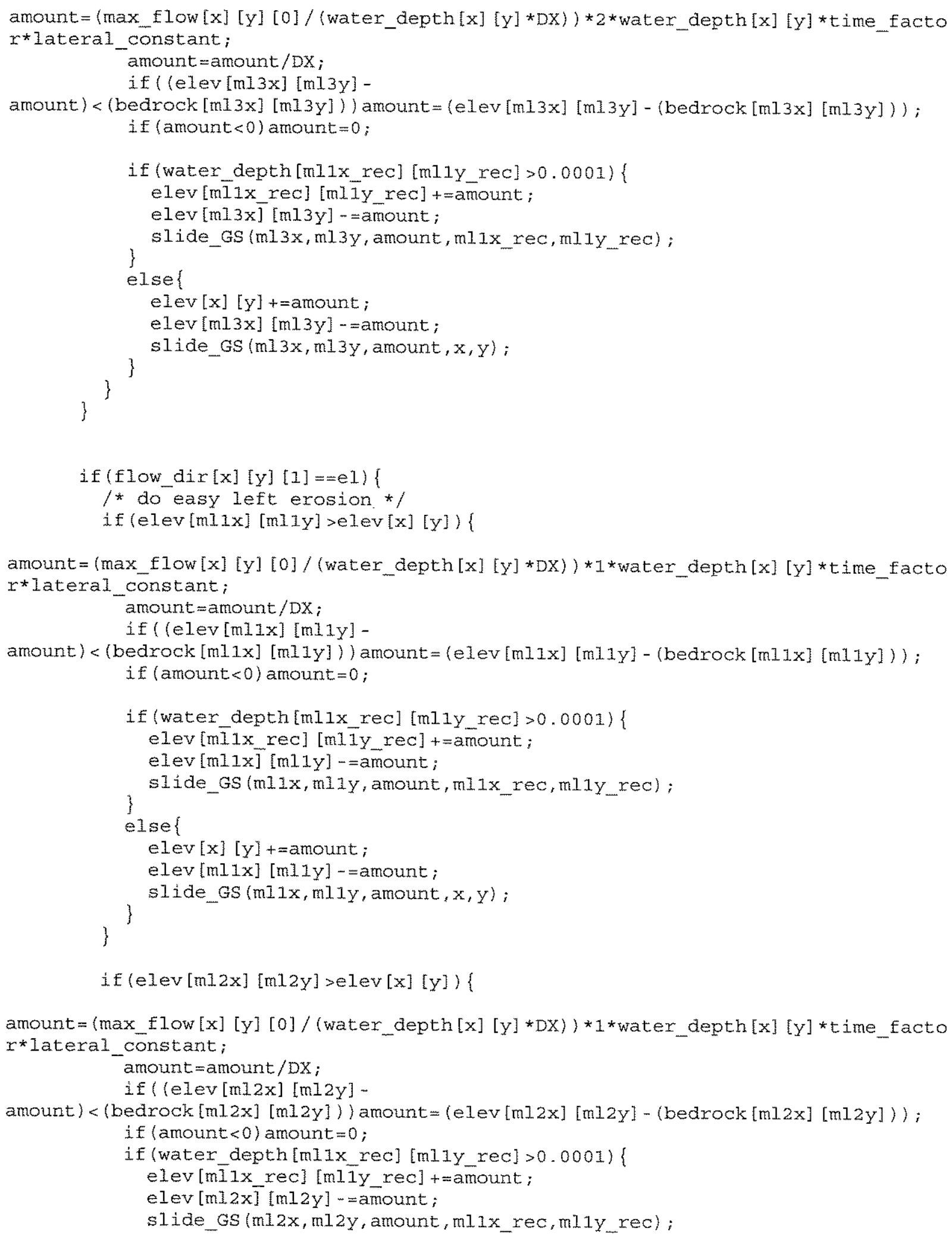




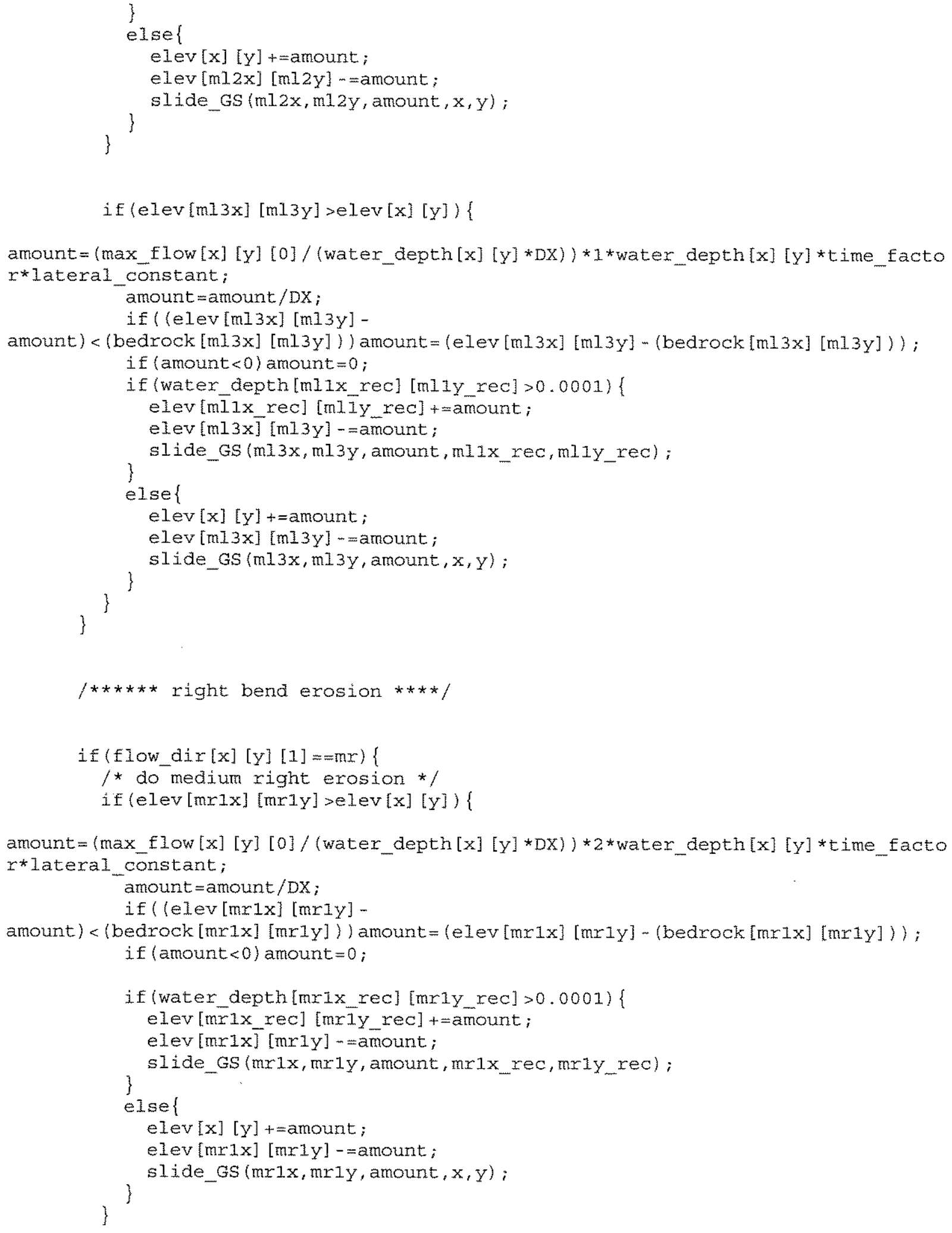


if (elev $[\mathrm{mr} 2 \mathrm{x}][\mathrm{m} r 2 \mathrm{y}]>\operatorname{elev}[\mathrm{x}][\mathrm{y}])\{$

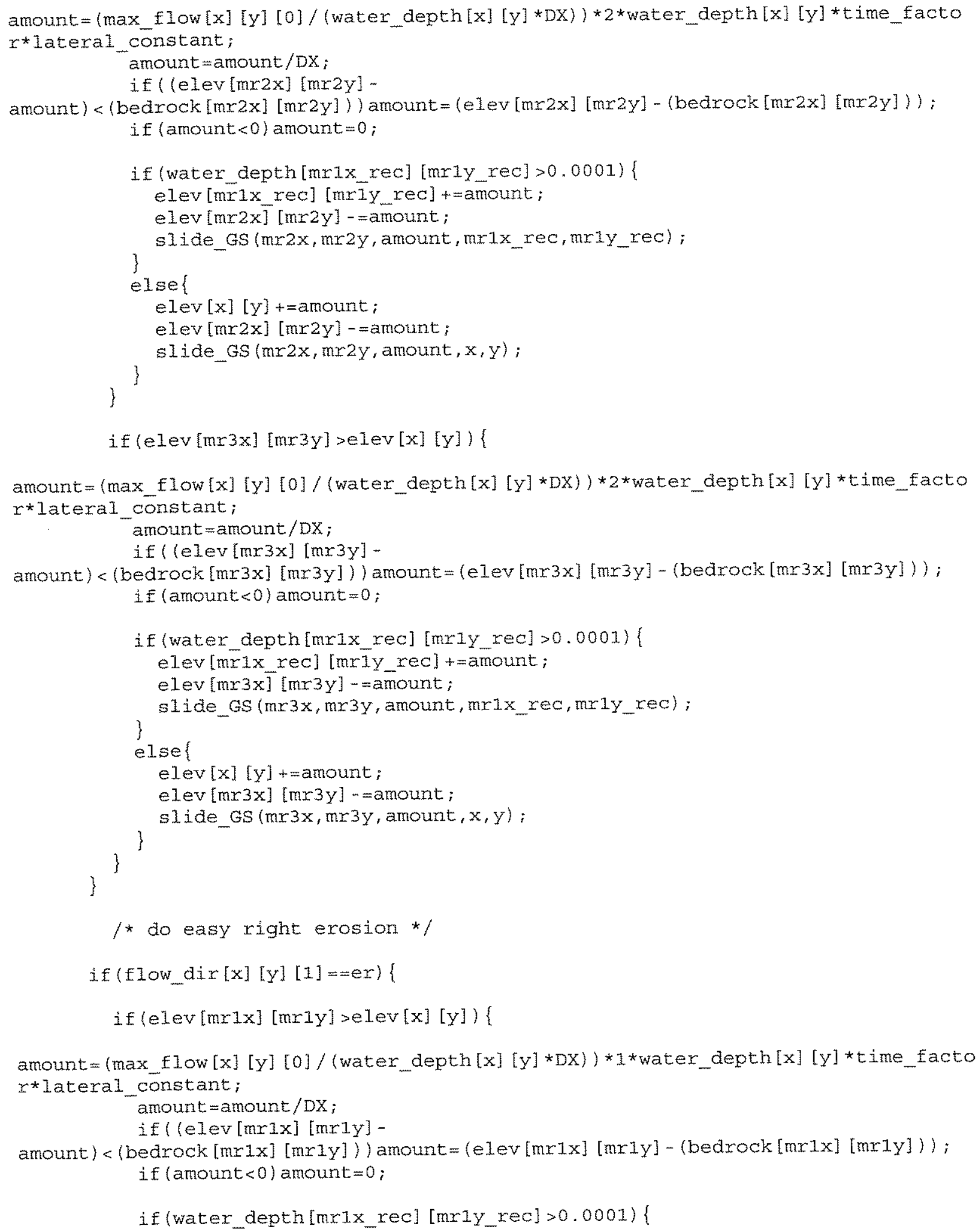




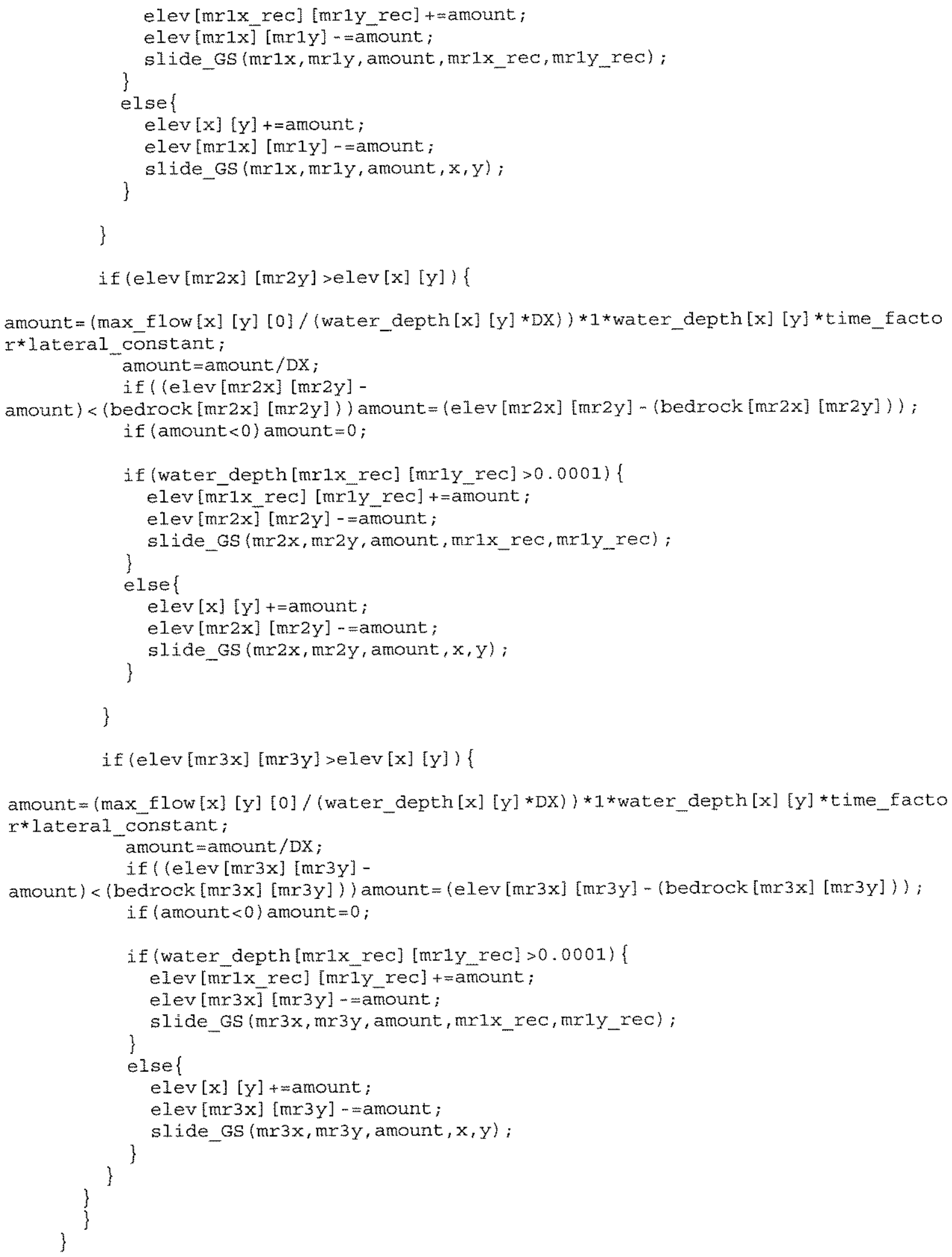




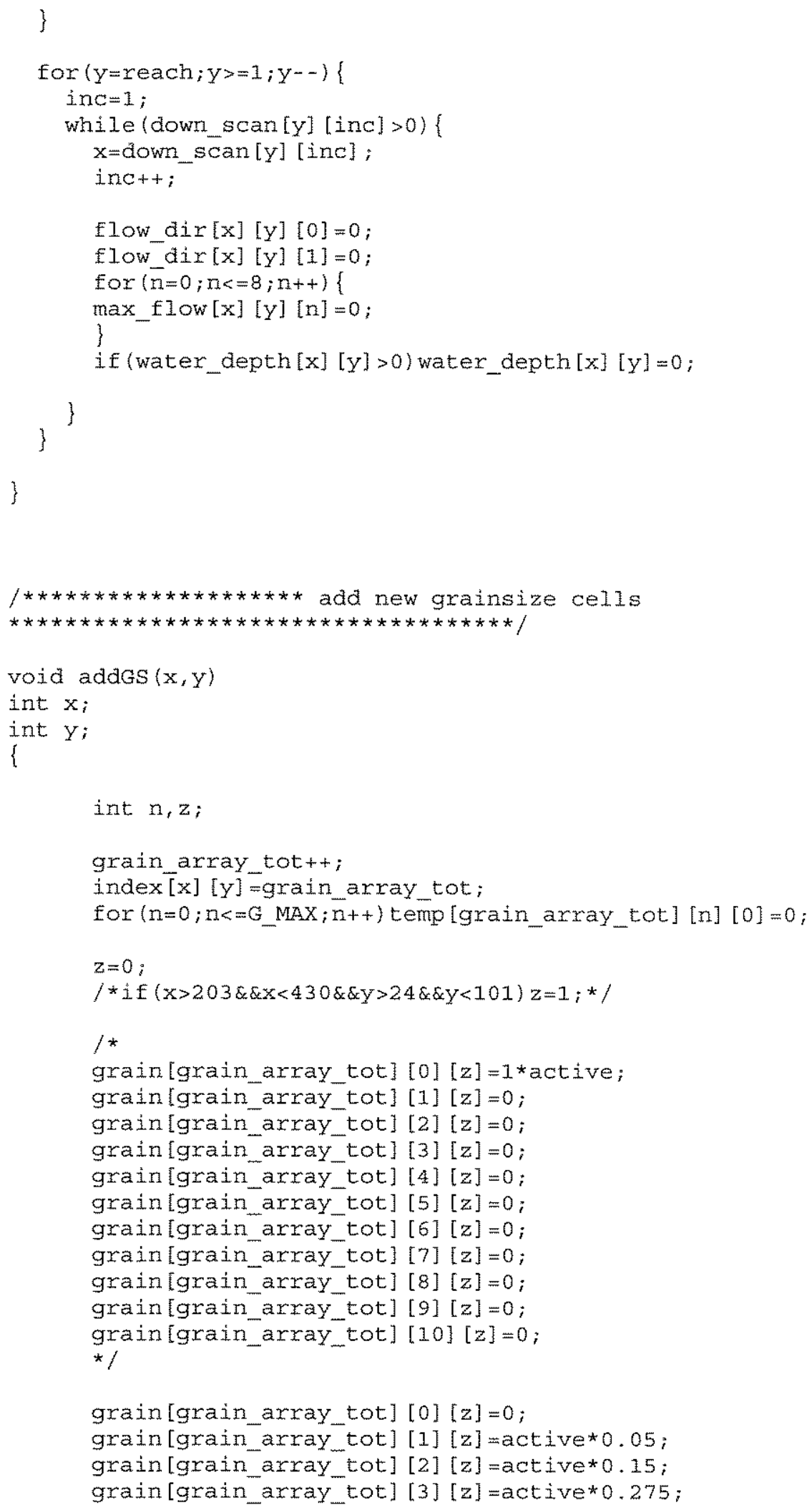




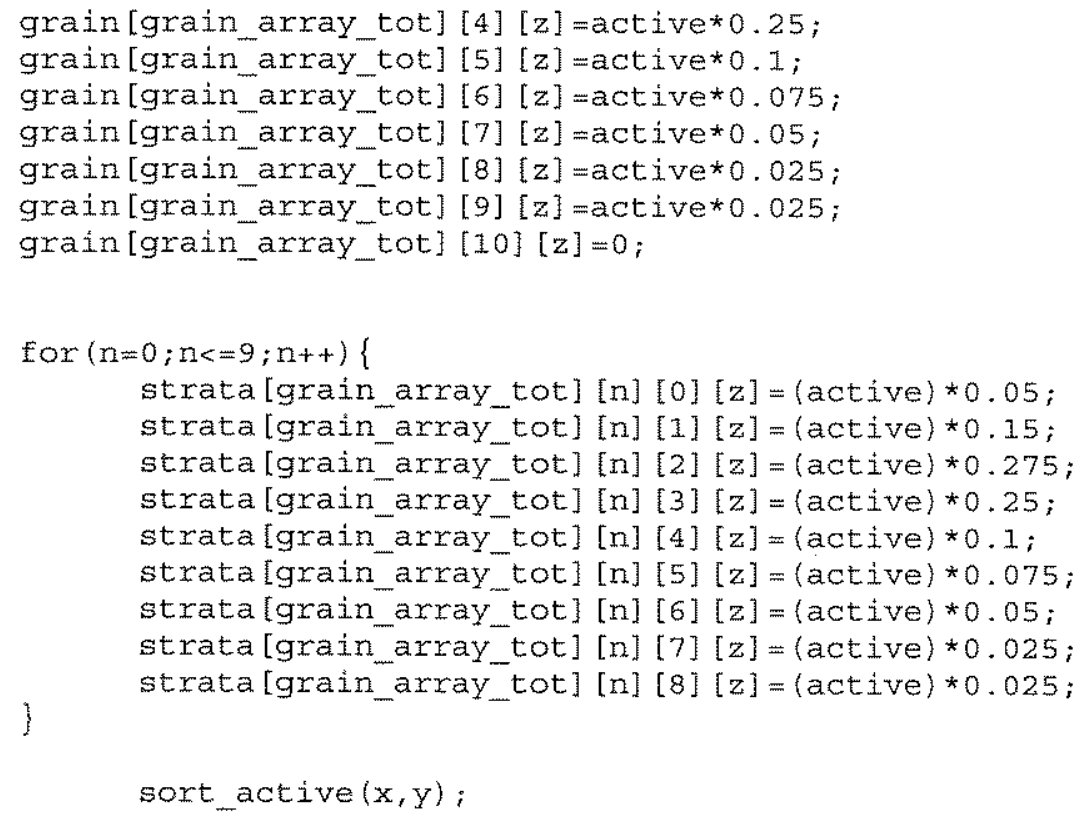




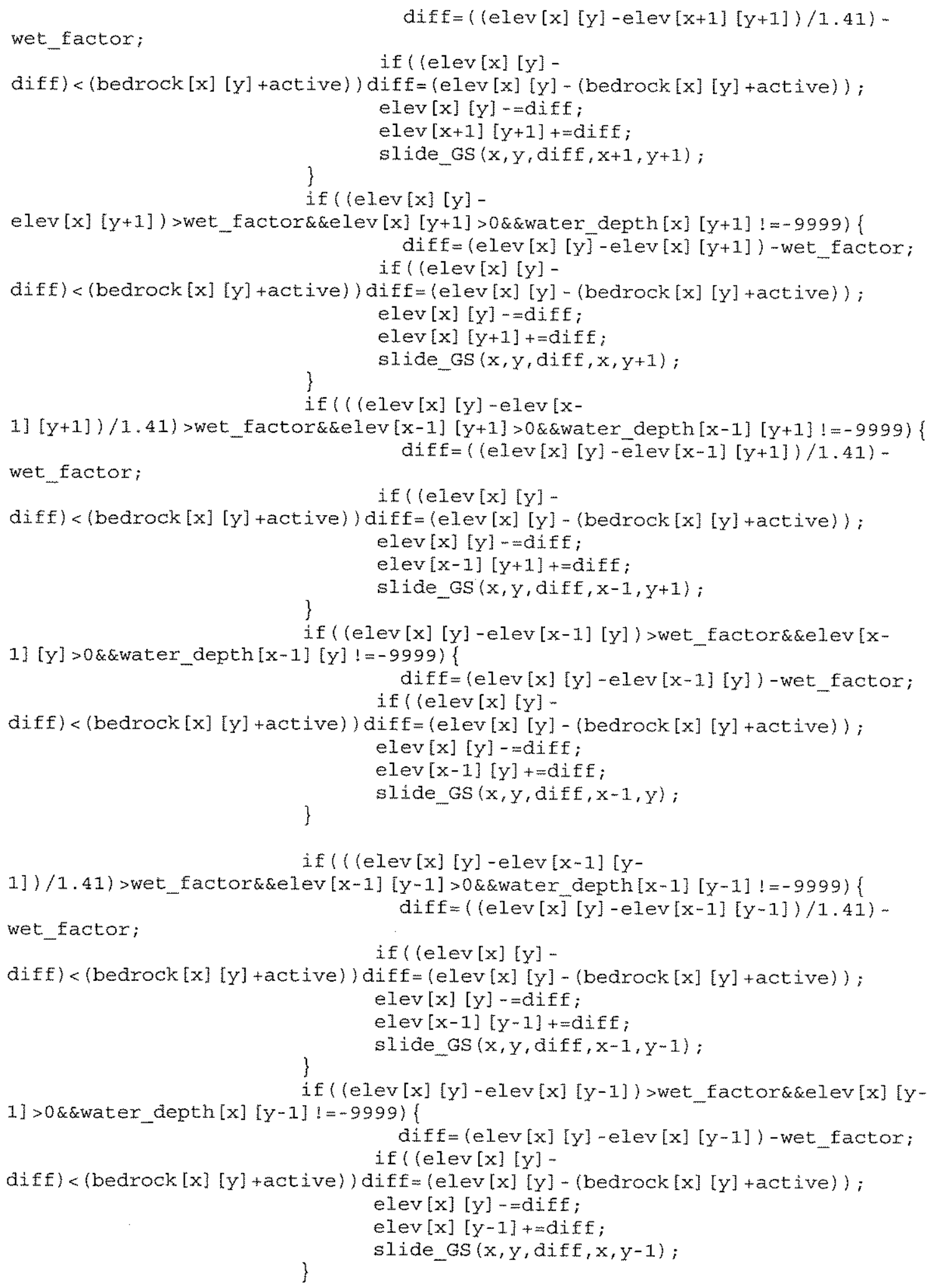


iff ( ( elev $[x][y]-e l e v[x+1][y-$

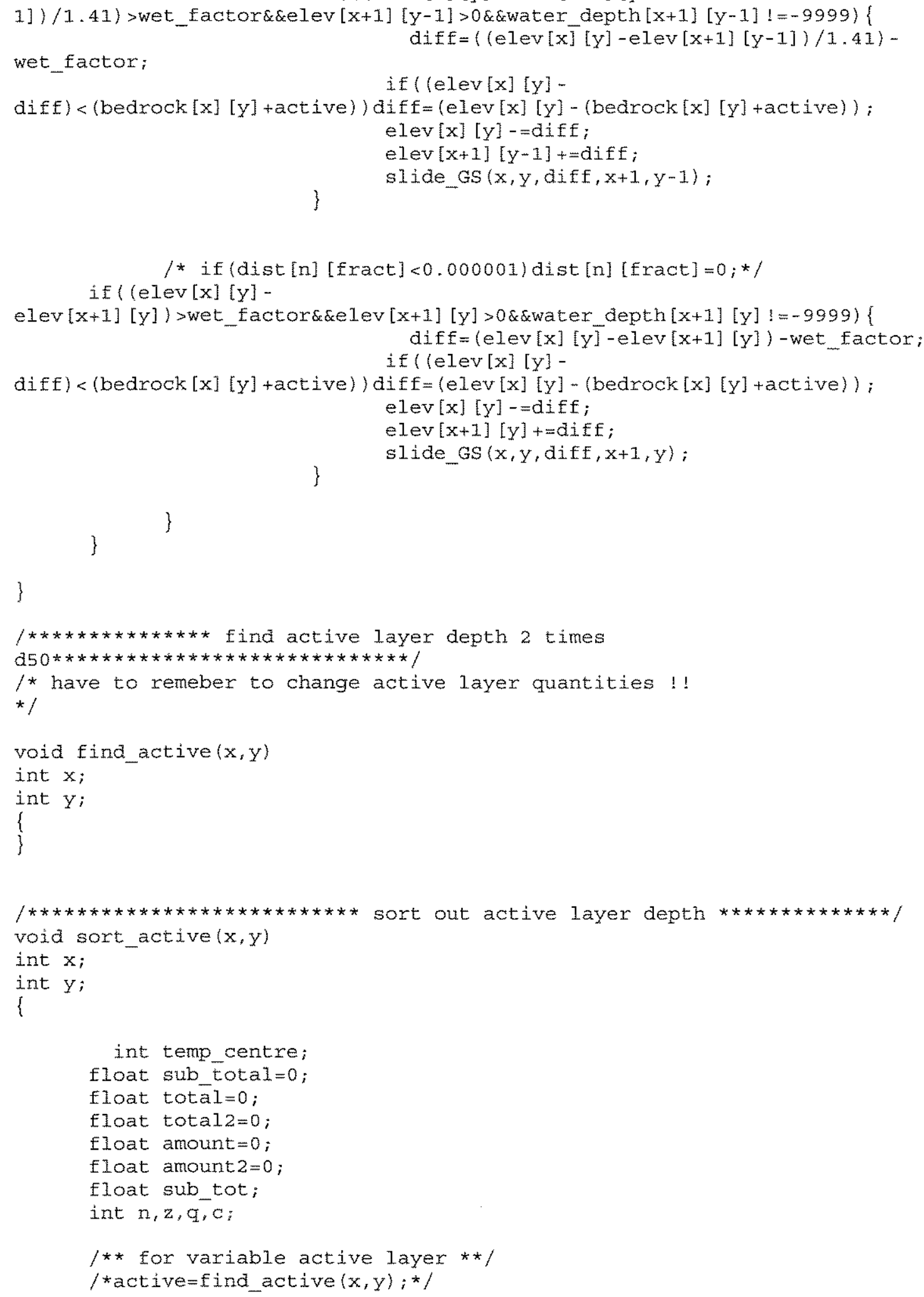




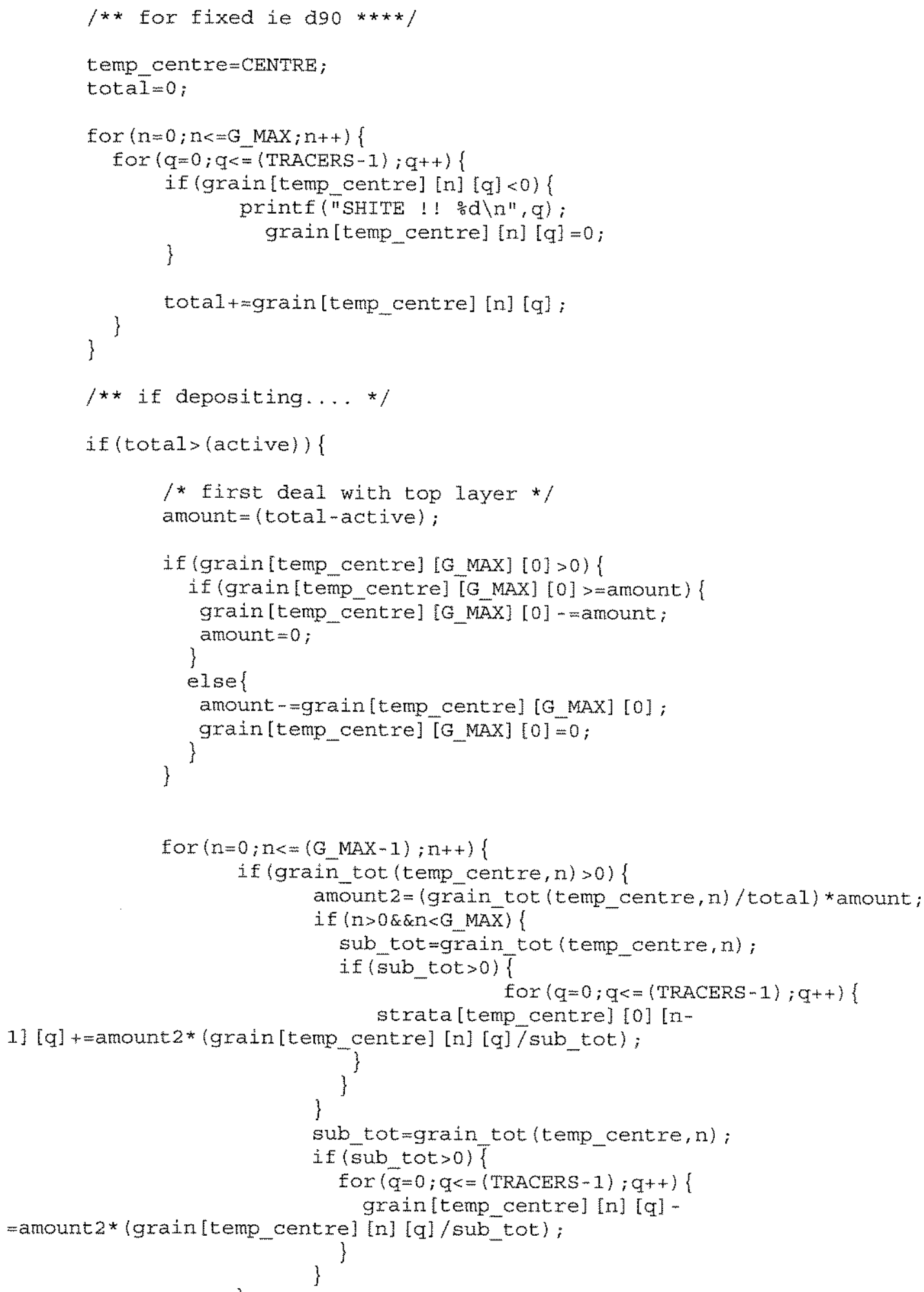


$/ *$ then workk down through the strata $* * /$

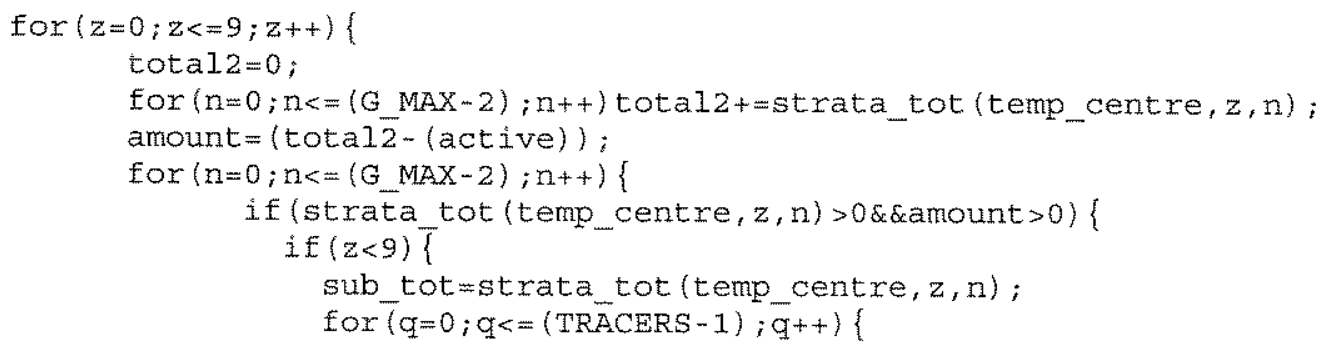




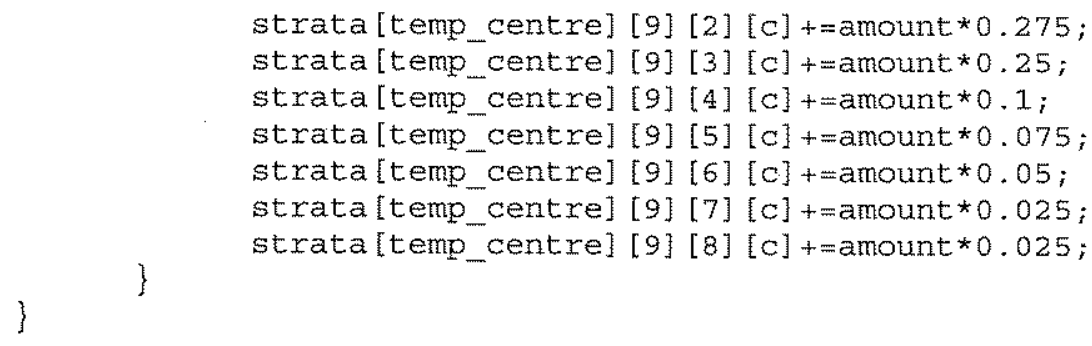

float four dp (inval)

float inval; 


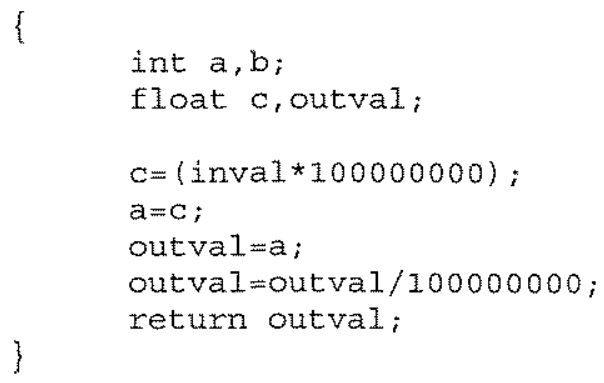




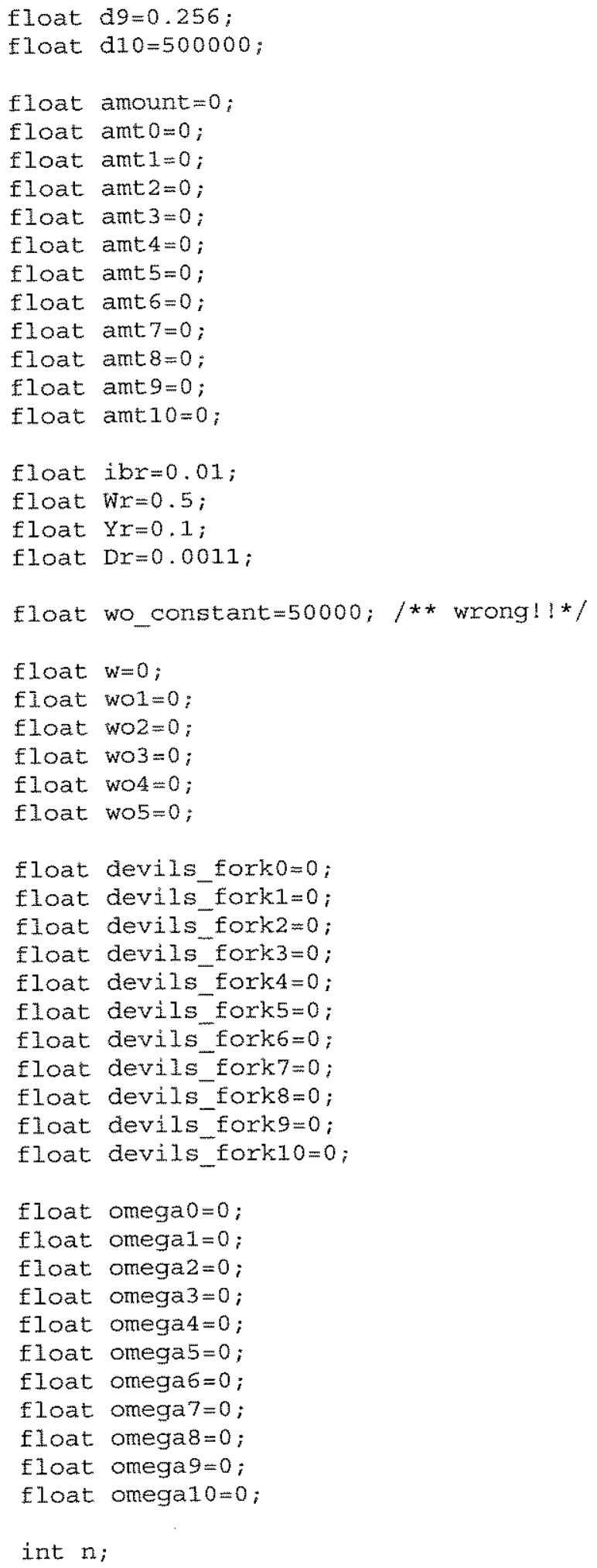


$\mathrm{V}=($ pow $($ depth, 0.67$)$ *pow $($ slope, 0.5$)) /$ manning

$/ * * *$ using Meyer Peter \& Muller */

$1 *$

if $(t>t$ crit5) amt $5=($ muller*pow $((t-t c r i t 5), 1.5))$;

iff $(t>t c r i t 4)$ amt $4=(\operatorname{muller} *$ pow $((t-t c r i t 4), 1.5))$;

if $(t>\operatorname{tarit} 3)$ amt $3=($ muller*pow $((t-t \operatorname{crit} 3), 1.5))$;

if $(t>t c r i t 2)$ amt $2=($ muller*pow $((t-t \operatorname{crit} 2), 1.5))$;

if $(t>\operatorname{tarit} 1)$ amt $1=(\operatorname{muller} \operatorname{pow}(t-\operatorname{tarit} 1), 1.5))$;

$* /$

/*** Using bagnold $1980 * * * * * * * * * * * * * * /$

$/ *$ assuming density of sed $=2250$ \& water $1000 * /$

/* this version gives weight, so divide by density 2250 to give volume */

$1 *$

$W=E * V ;$

wo1 $1=$ wo constant*pow $(d 1,1.5) * \log ((12 * \operatorname{depth} / \mathrm{d} 1))$;

wo2 $=$ wo constant $*$ pow $(\mathrm{d} 2,1.5) * \log ((12 * \operatorname{depth} / \mathrm{d} 2))$;

wo3 =wo_constant $*$ pow $(d 3,1.5) * \log ((12 *$ depth/d3));

wo $4=$ wo_constant*pow $(\mathrm{d} 4,1.5) * \log ((12 * \operatorname{depth} / \mathrm{d} 4))$;

wo5 $=$ wo_constant $*$ pow $(d 5,1.5) * \log ((12 * \operatorname{depth} /$ d5 $))$;

主 $(w>$ wo 1$) \operatorname{amt} 1=(1.8 * 0.5 *$ pow $(((w-w o 1) / W r), 1.5) *$ pow $(($ depth $/ Y r),-$ $0.6667) *$ pow $((d I / D r),-0.5)) / 2250 ;$

if $(w>$ wo2 $)$ amt $2=(1.8 * 0.5 *$ pow $(((w-w 02) / W r), 1.5) *$ pow $(($ depth $/$ Yr $)$, -

$0.6667) *$ pow $((\mathrm{d} 2 / \mathrm{Dr}),-0.5)) / 2250$

if $(w>$ wo3 $)$ amt $3=(1.8 * 0.5 *$ pow $(((w-w o 3) / W r), 1.5) *$ pow $(($ depth $/$ Yr $)$, -

$0.6667) *$ pow $((\mathrm{d} 3 / \mathrm{Dr}),-0.5)) / 2250$

if $(w>w 04)$ amt $4=(1.8 * 0.5 *$ pow $((w-w 04) / w r), 1.5) *$ pow $((\operatorname{depth} / Y r)$, -

$0.6667) *$ pow $((d 4 /$ Dr $),-0.5)) / 2250$;

if $(w>$ wo5 $)$ amt $5=(1.8 * 0.5 *$ pow $(((w-w 05) / W r), 1.5) *$ pow $((\operatorname{depth} / Y r)$, -

$0.6667) *$ pow $((d 5 /$ Dr $),-0.5)) / 2250$;

* $/$

$/ * * * * * * * * *$ using einstein brown $1950 * * * * * * * * * /$

$/ *$ assuming depth $=R * /$

/* all taken from steves fluid book */

devils_forko $=((2250-1000) *$ do $) /(1000 *$ depth*slope $)$;

devils_fork $1=((2250-1000) * \mathrm{~d} 1) /(1000 *$ depth*slope $)$;

devils fork $2=((2250-1.000) *$ d2 $) /(1000 *$ depth*slope $)$;

devils_fork $3=((2250-1000) *$ d 3$) /(1000 *$ depth*slope $)$;

devils_fork $4=((2250-1000) * \mathrm{~d} 4) /(1000 *$ depth*slope $)$;

devils fork $5=((2250-1000) *$ d5 $) /(1000 *$ depth*slope $)$;

devils_fork6 $=((2250-1000) * d 6) /(1000 *$ depth*slope);

devils_fork $7=((2250-1000) * d 7) /(1000 *$ depth*slope $)$;

devils fork $8=((2250-1000) *$ d8 $) /(1000 *$ depth*slope $)$;

devils_fork $9=((2250-1000) *$ d9 $) /(1000 *$ depth*slope $)$;

devils_fork10 $=((2250-1000) *$ d10 $) /(1000 *$ depth*slope $)$;

omega $0=40 *$ pow $((1 /$ devils fork 0$), 3)$;

omega $1=40 *$ pow $((1 /$ devils_fork 1$), 3)$; 

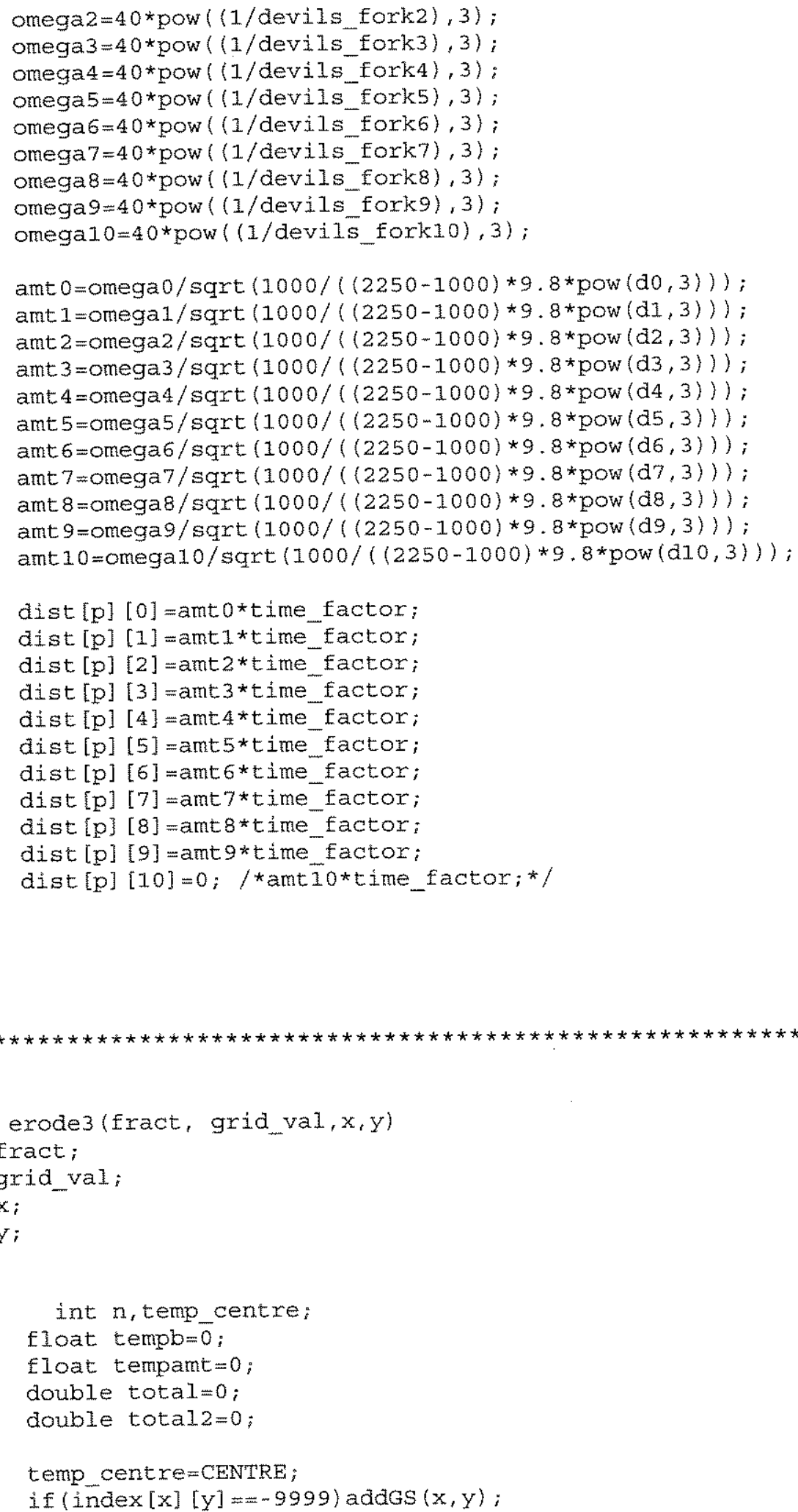


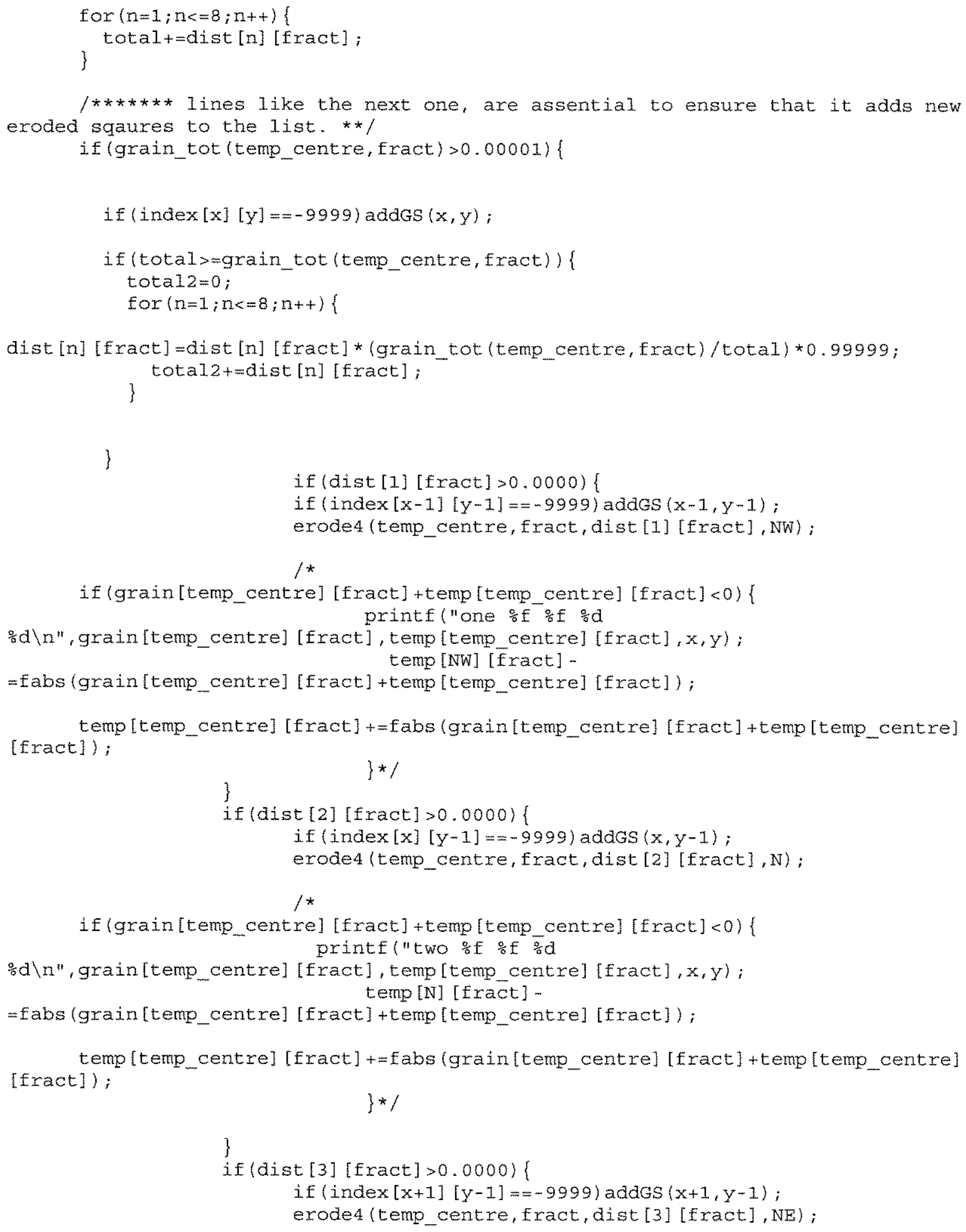




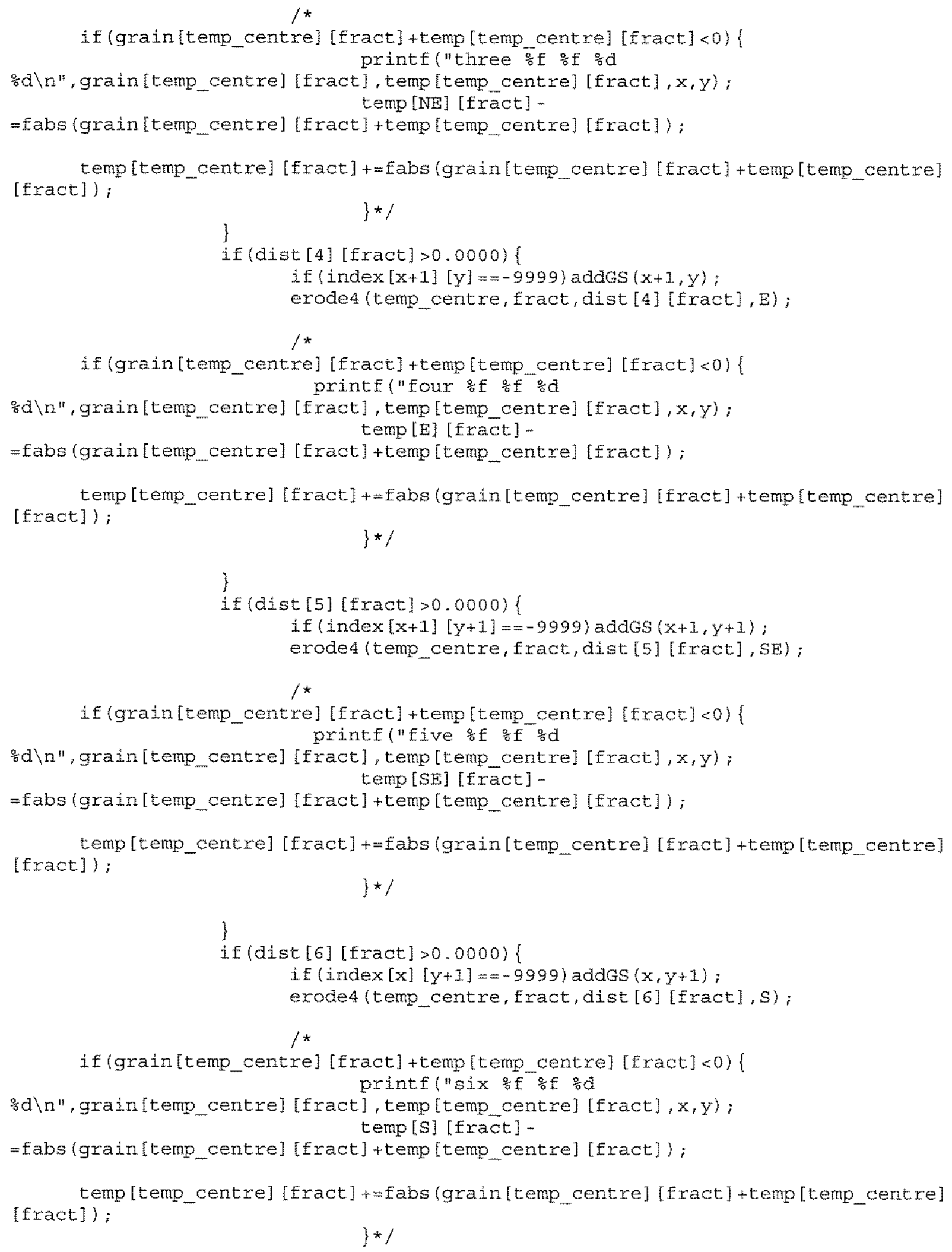




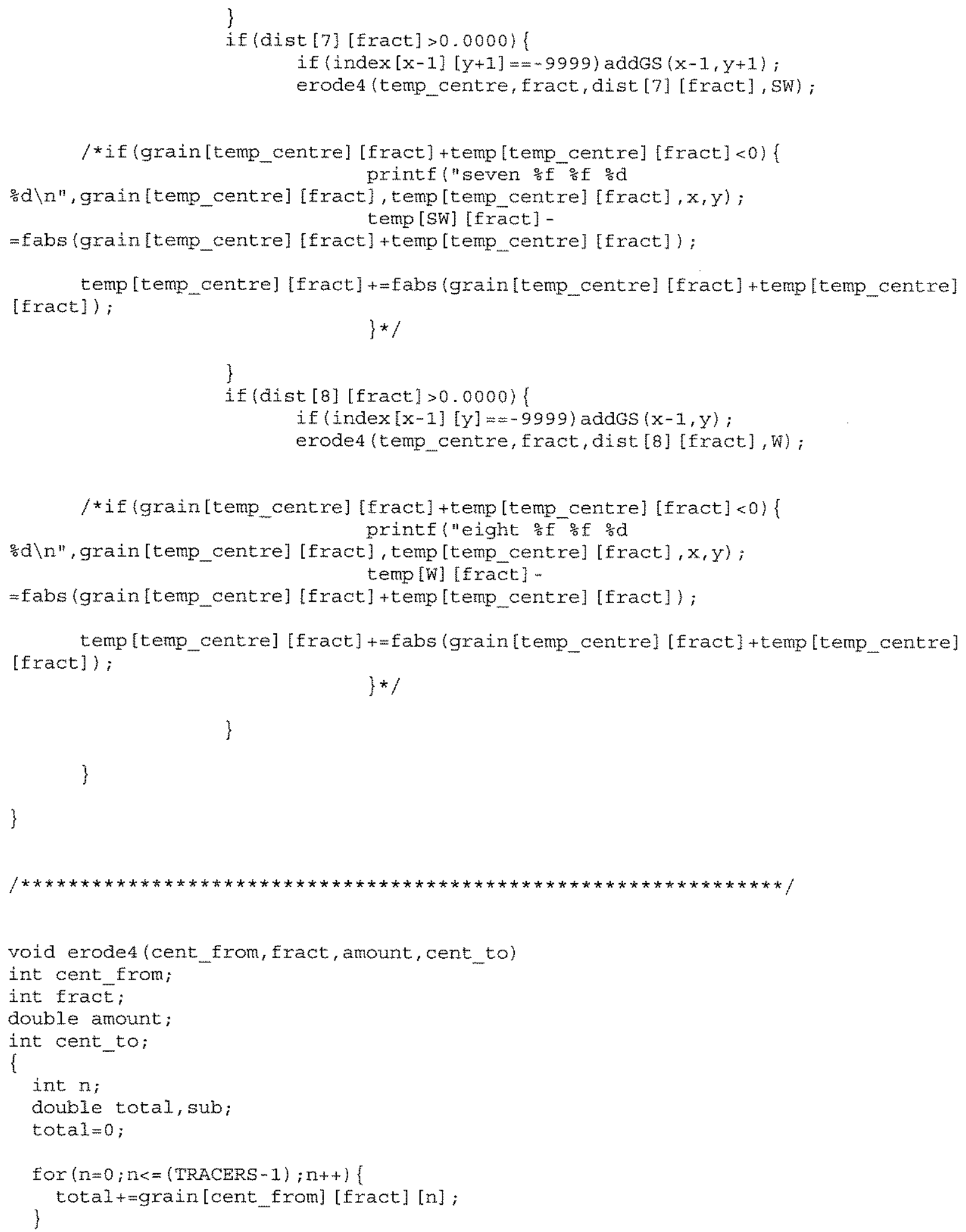




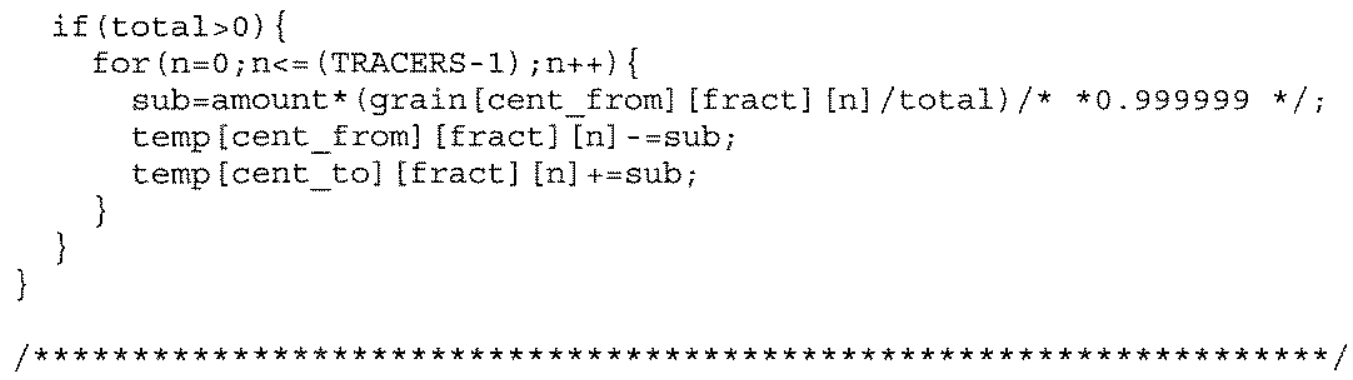




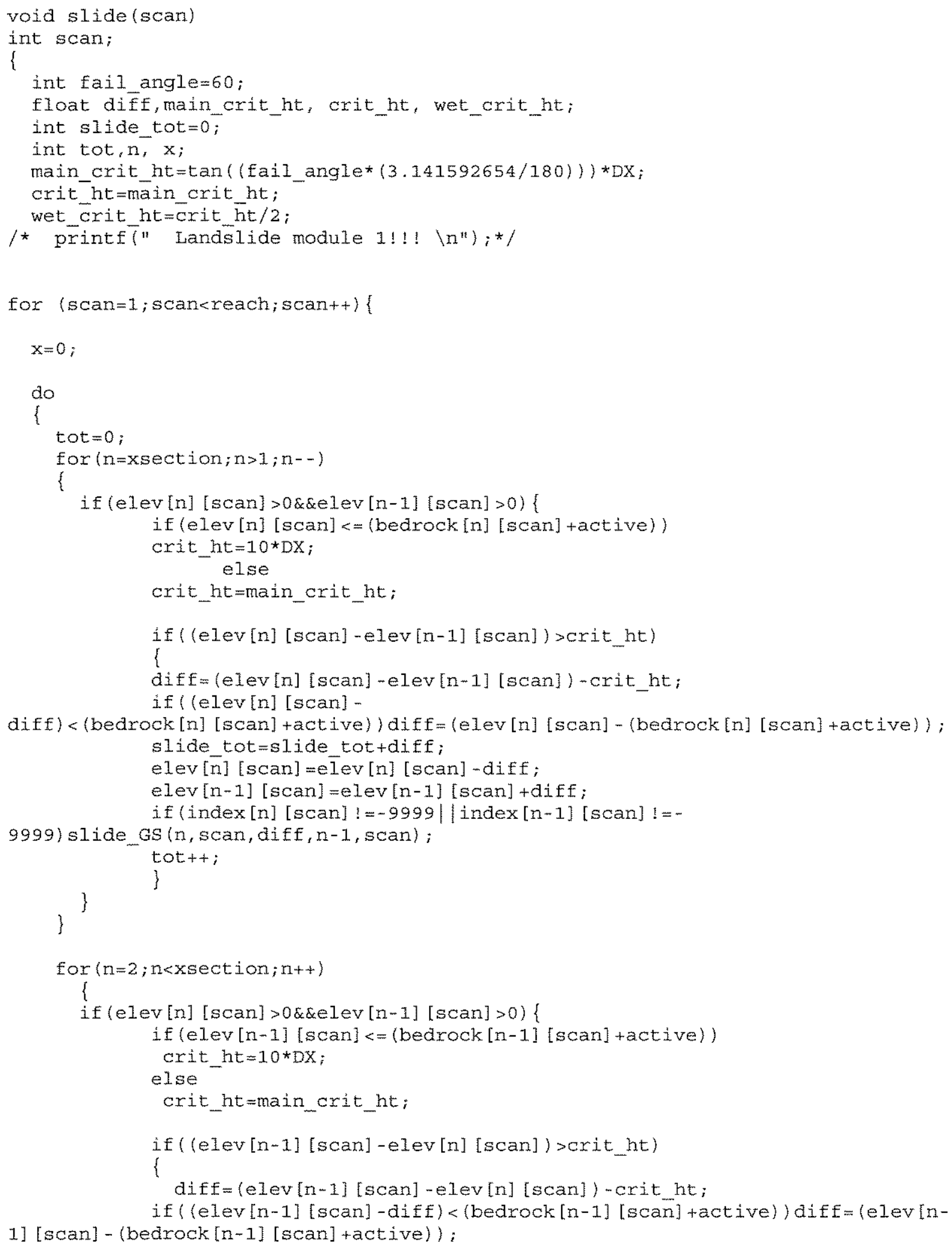




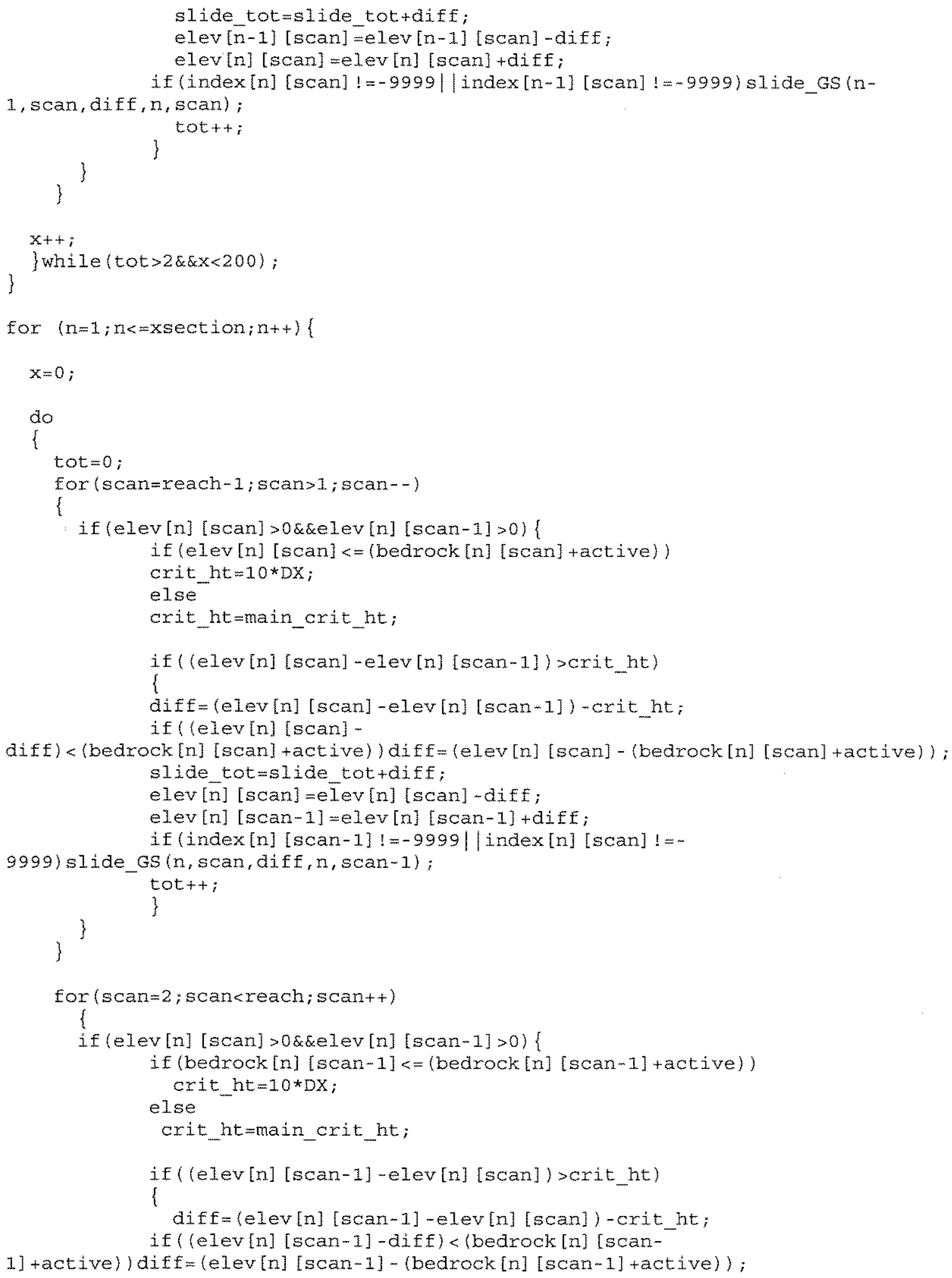




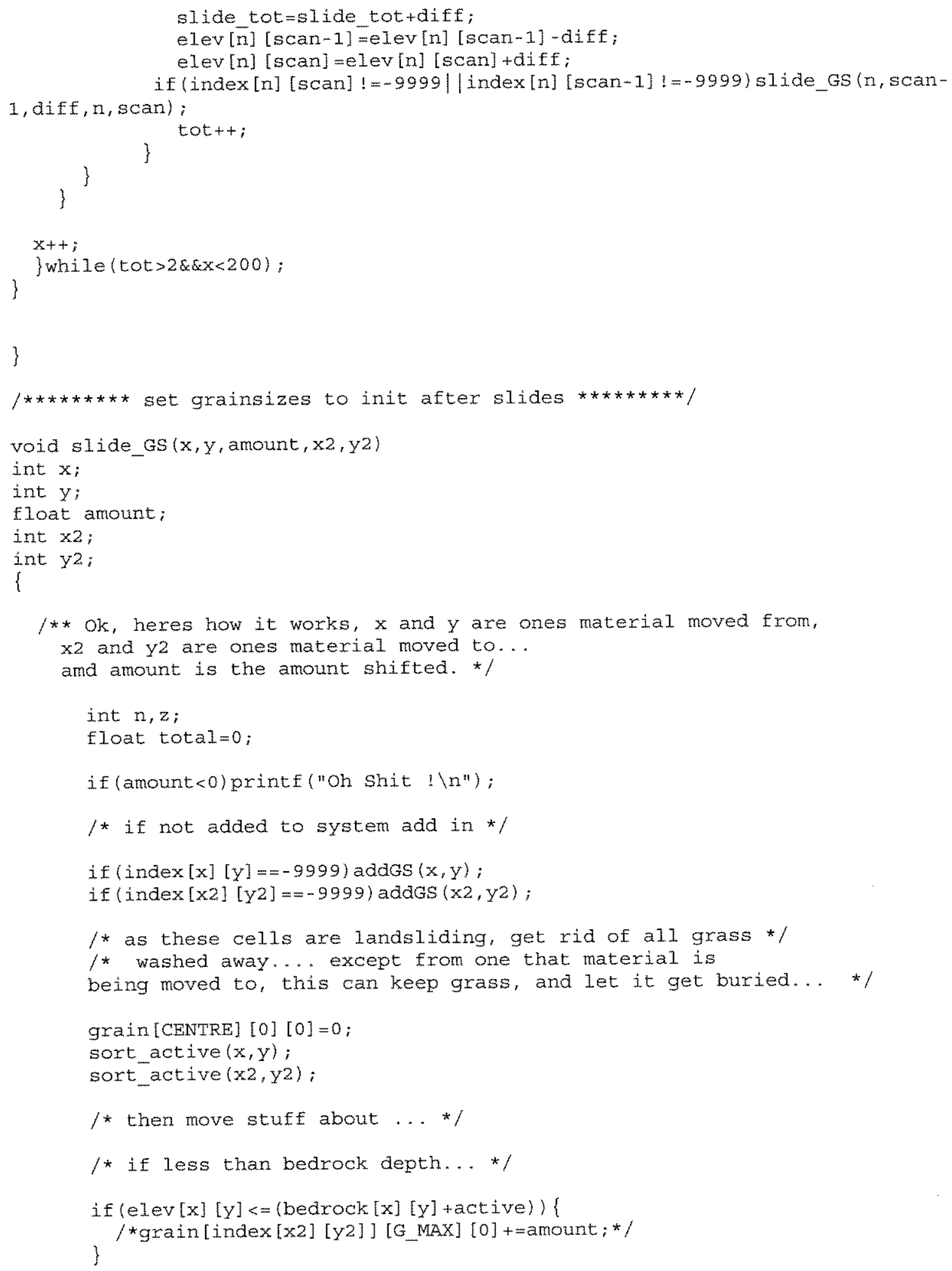




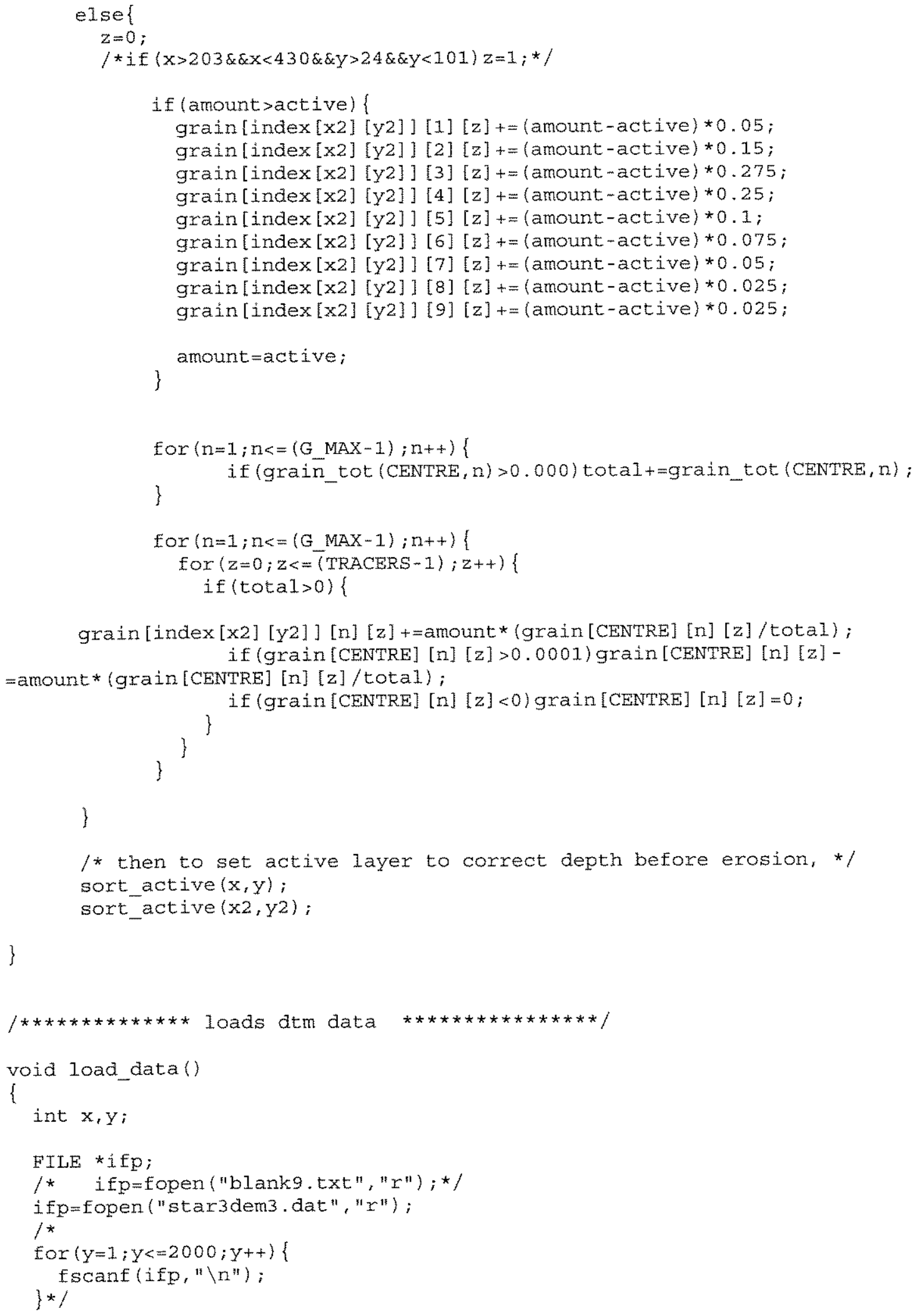




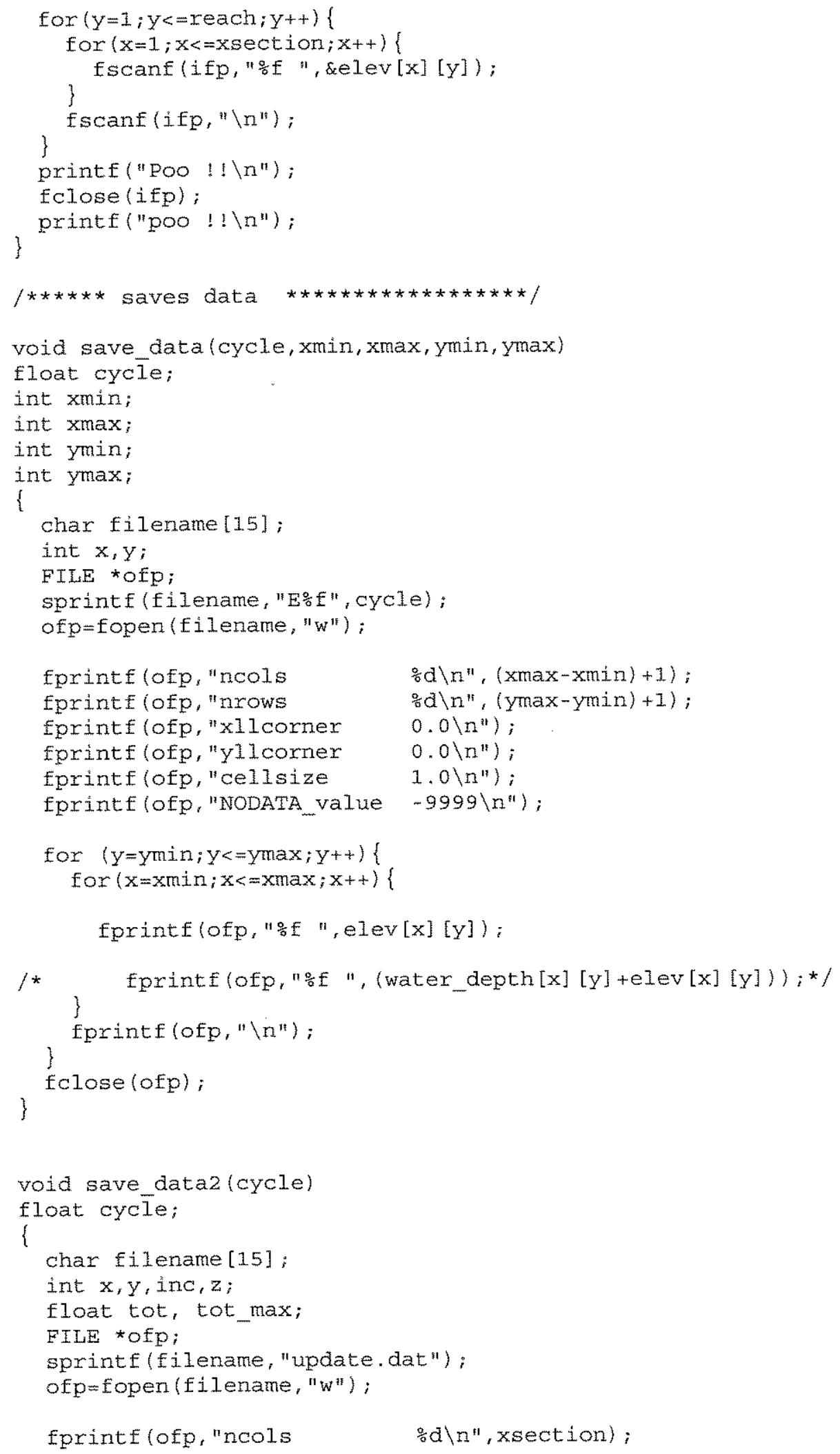




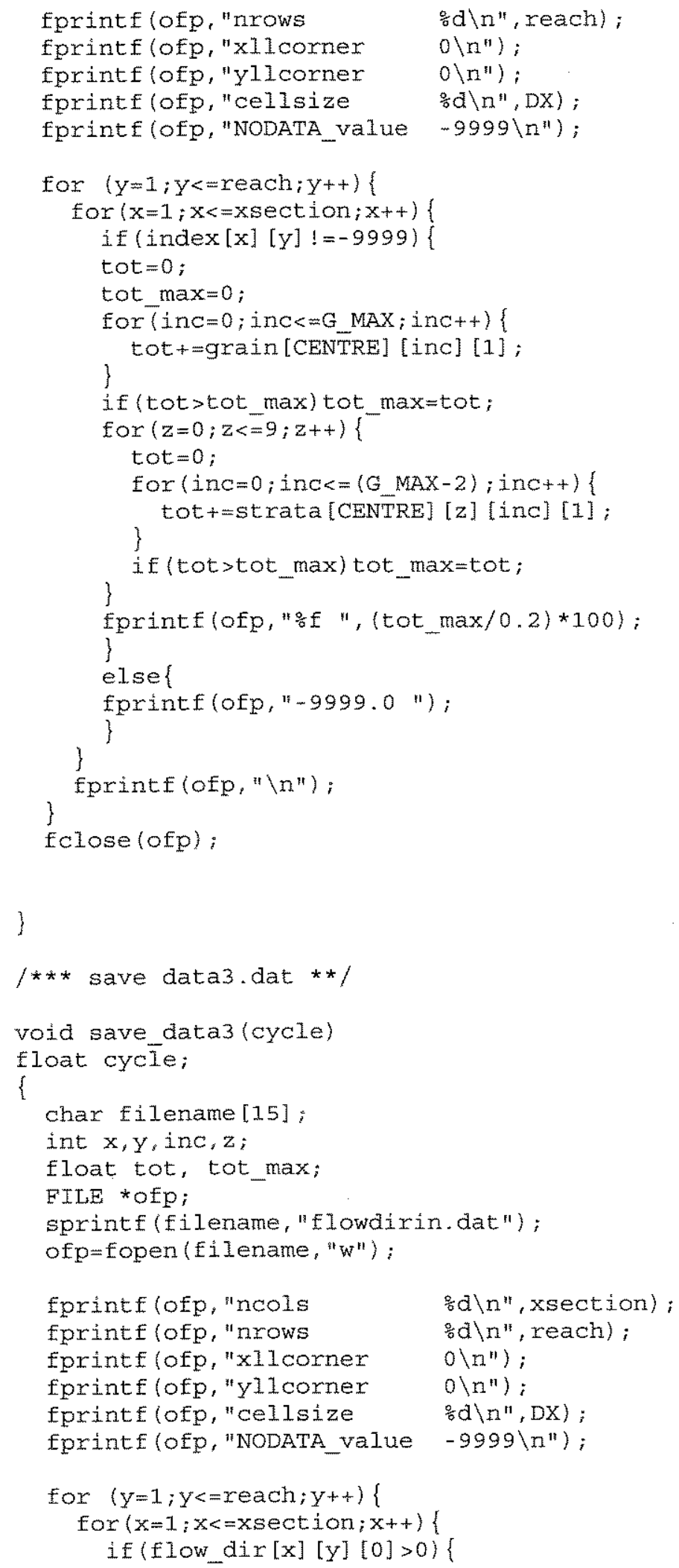




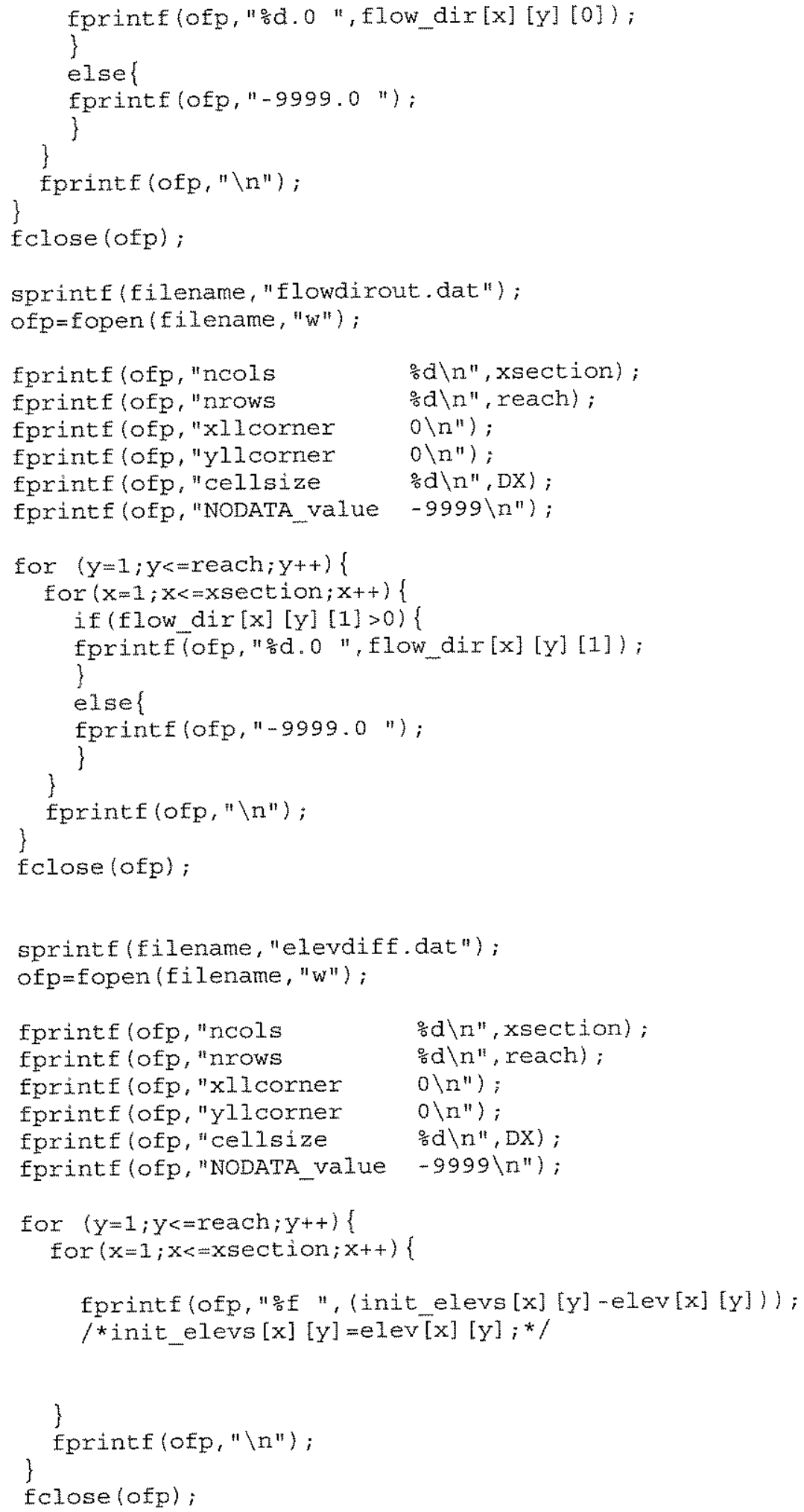




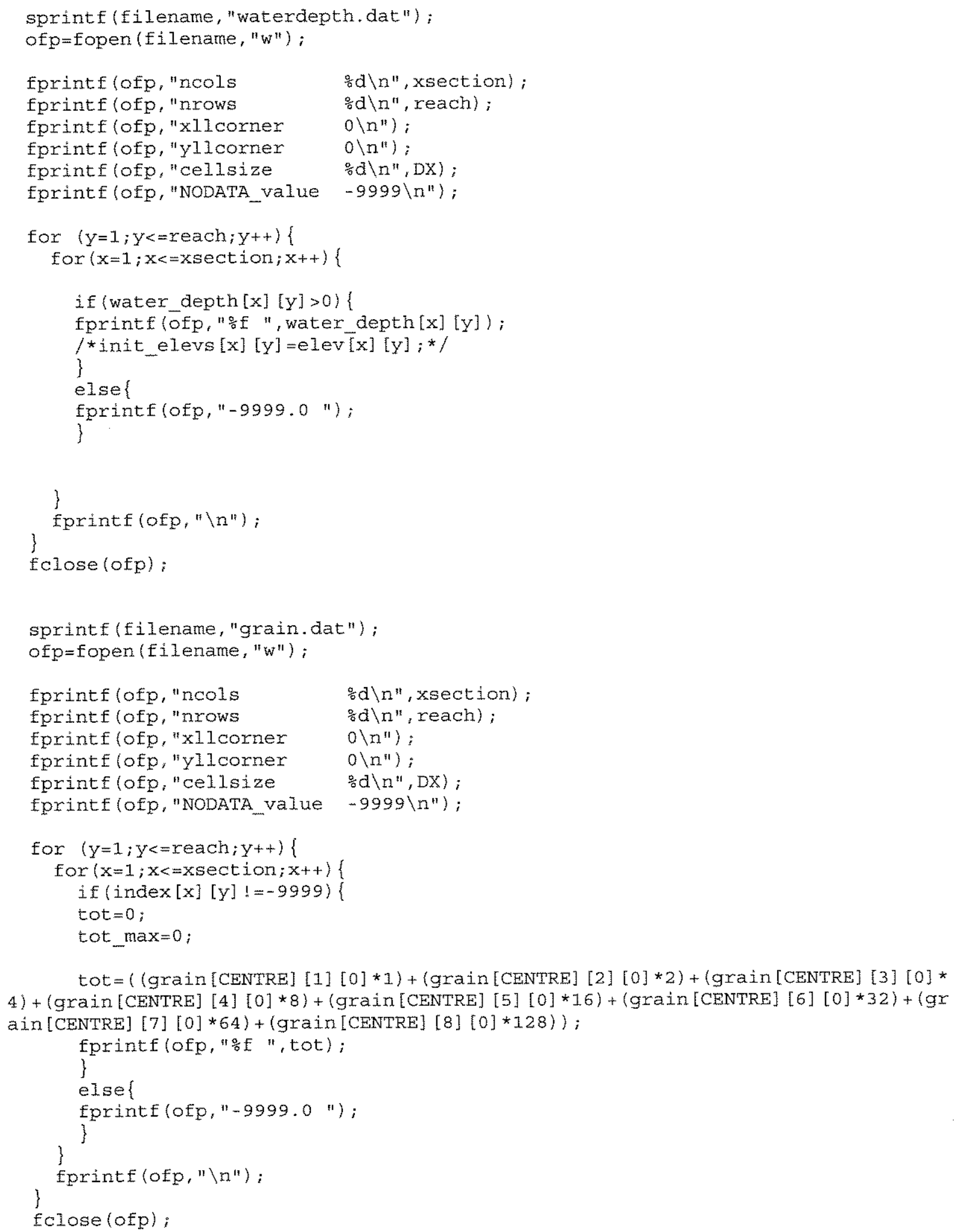




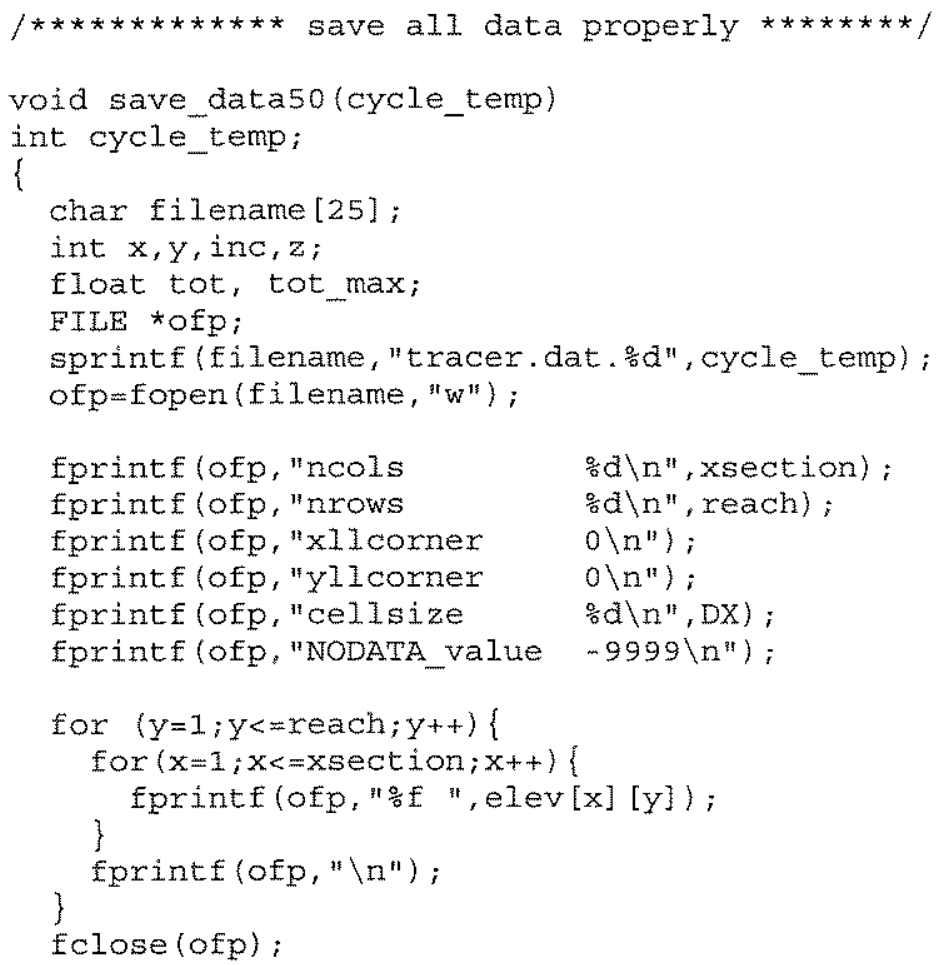




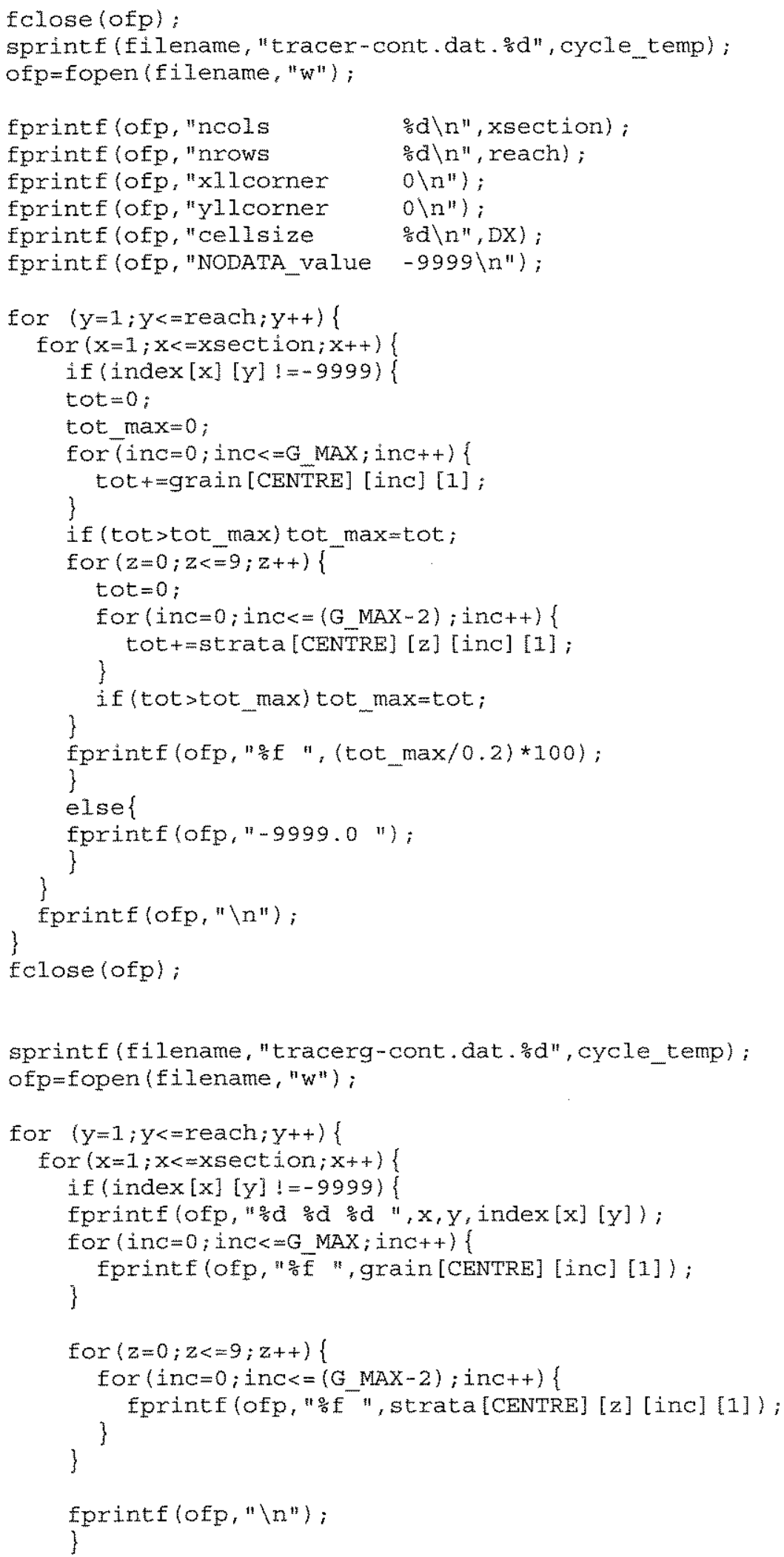




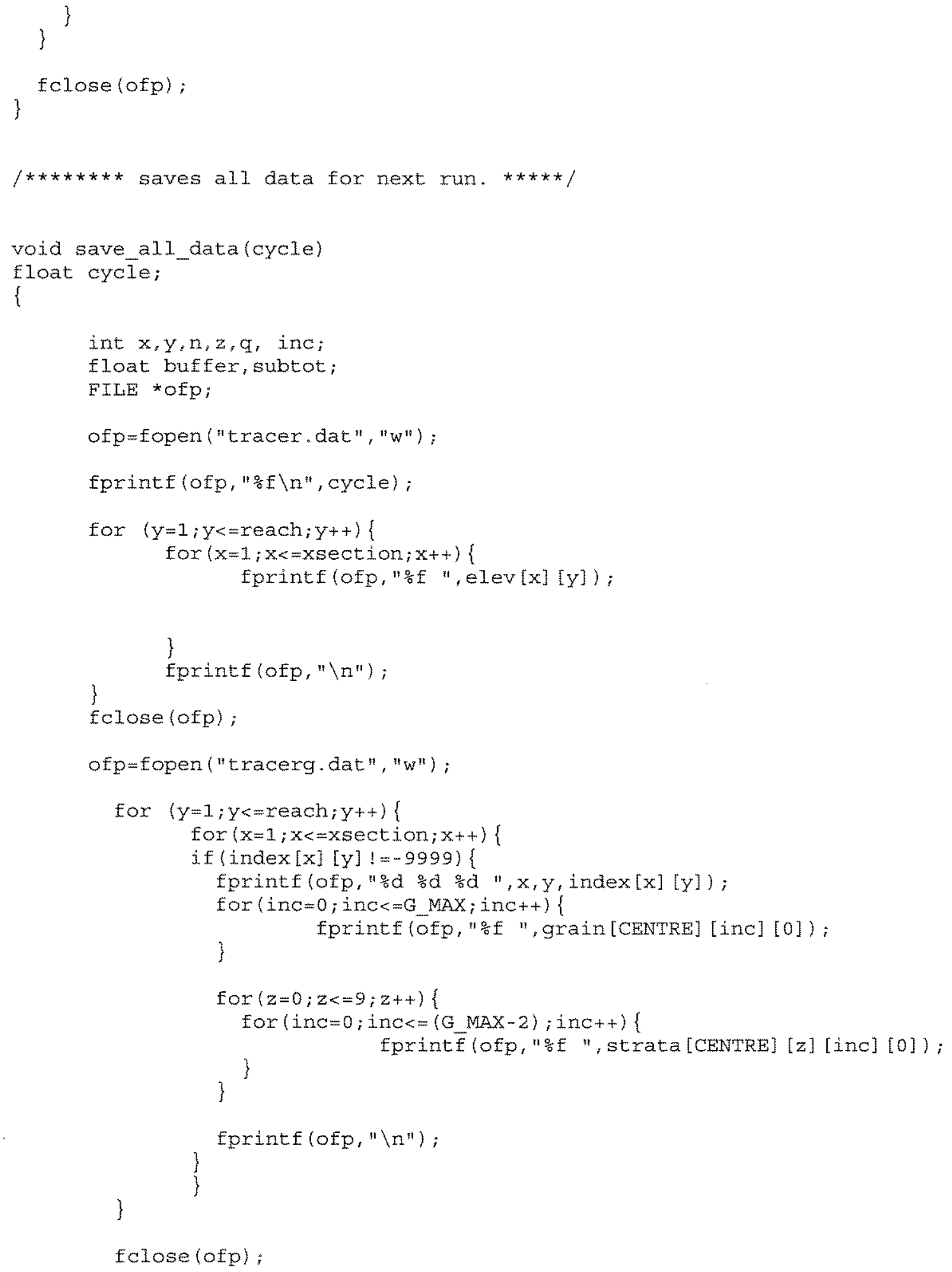




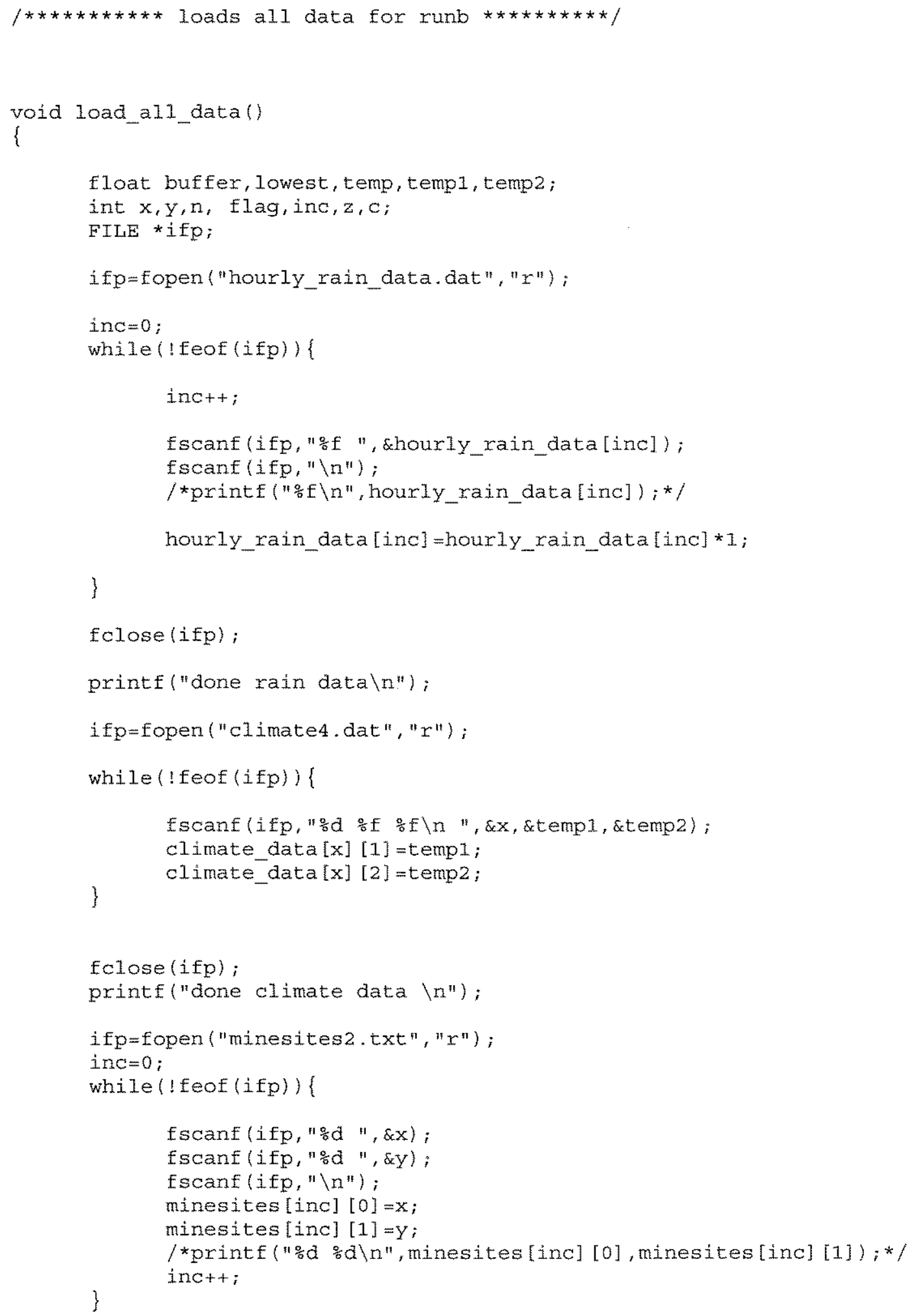




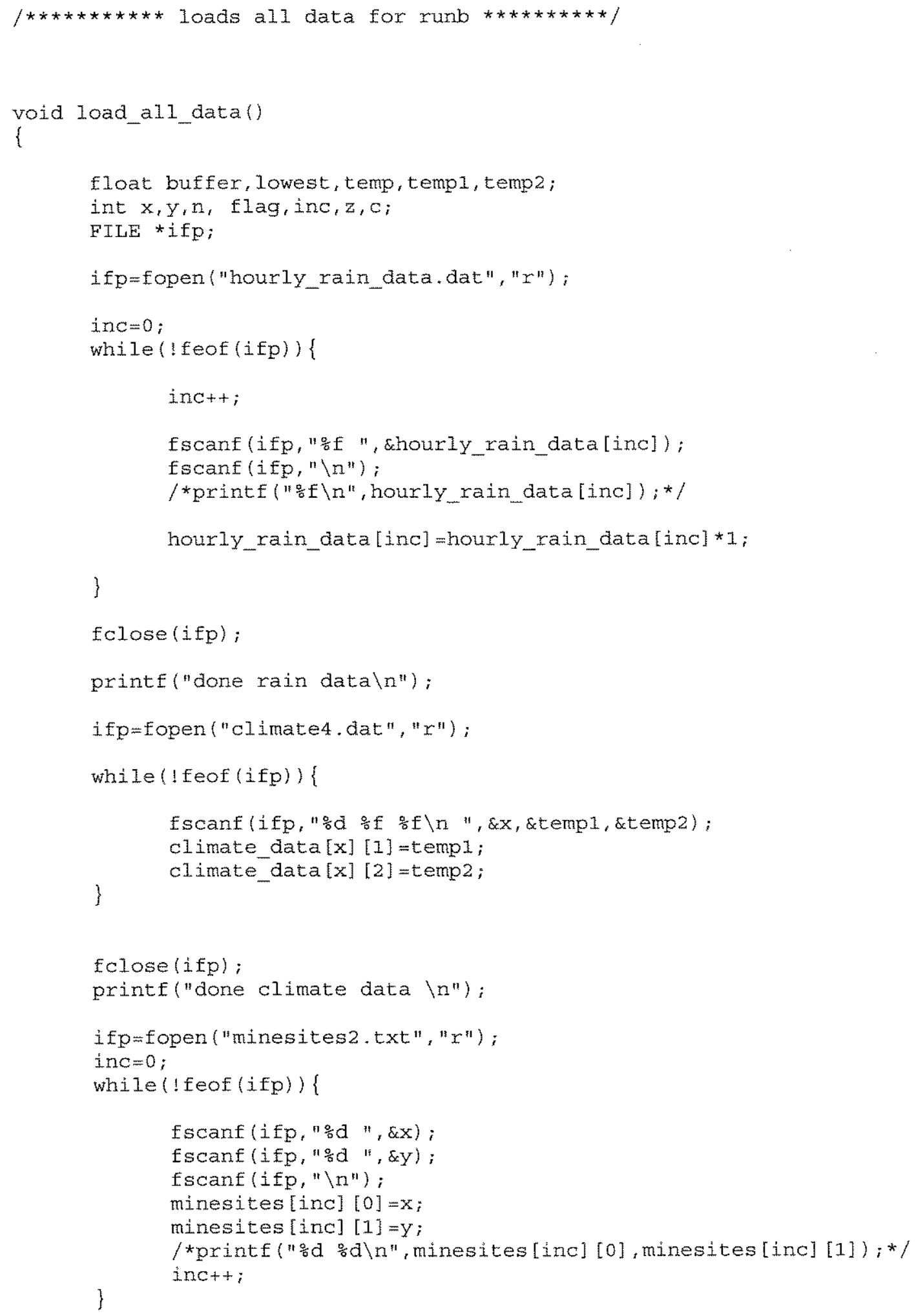




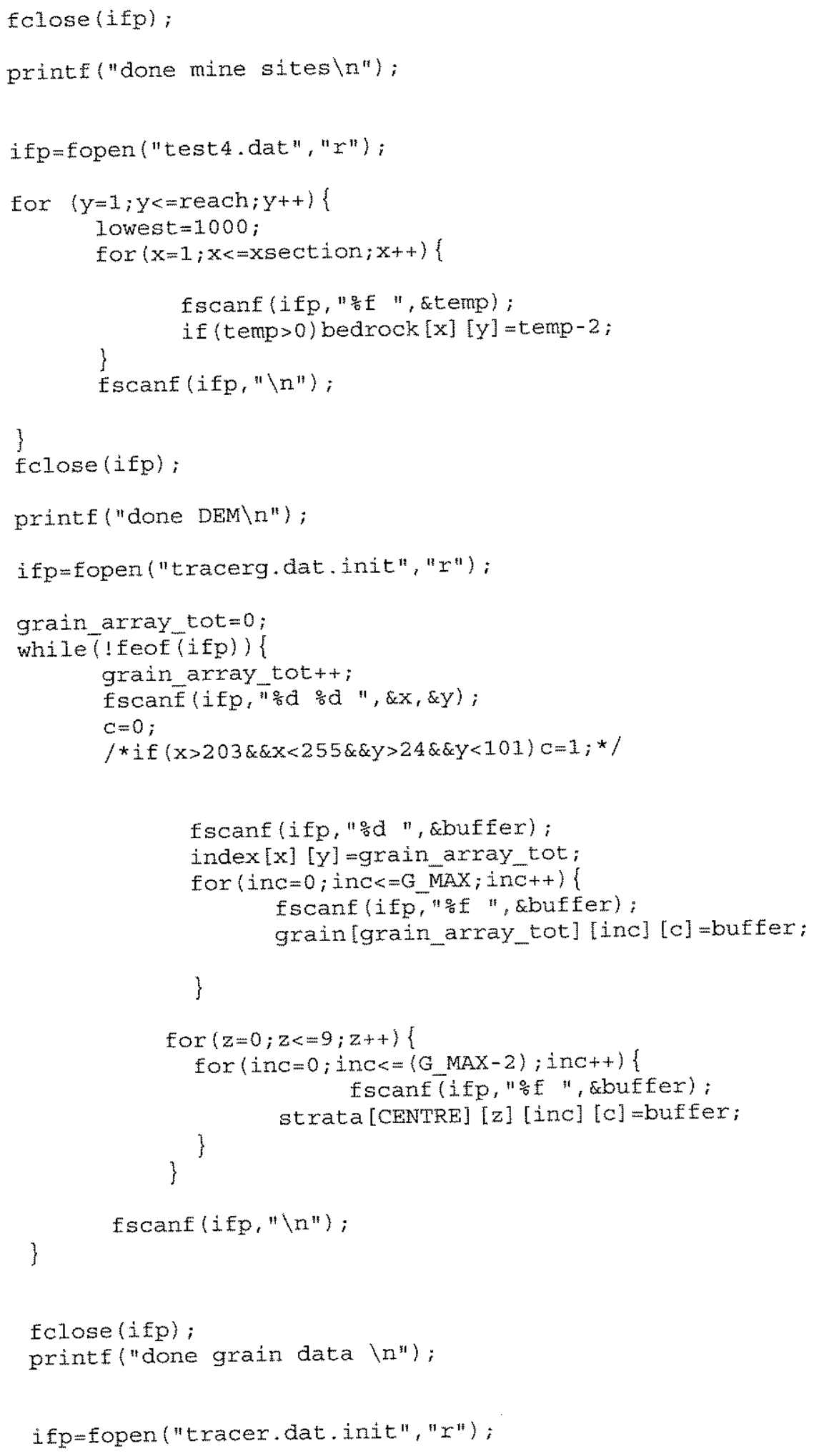




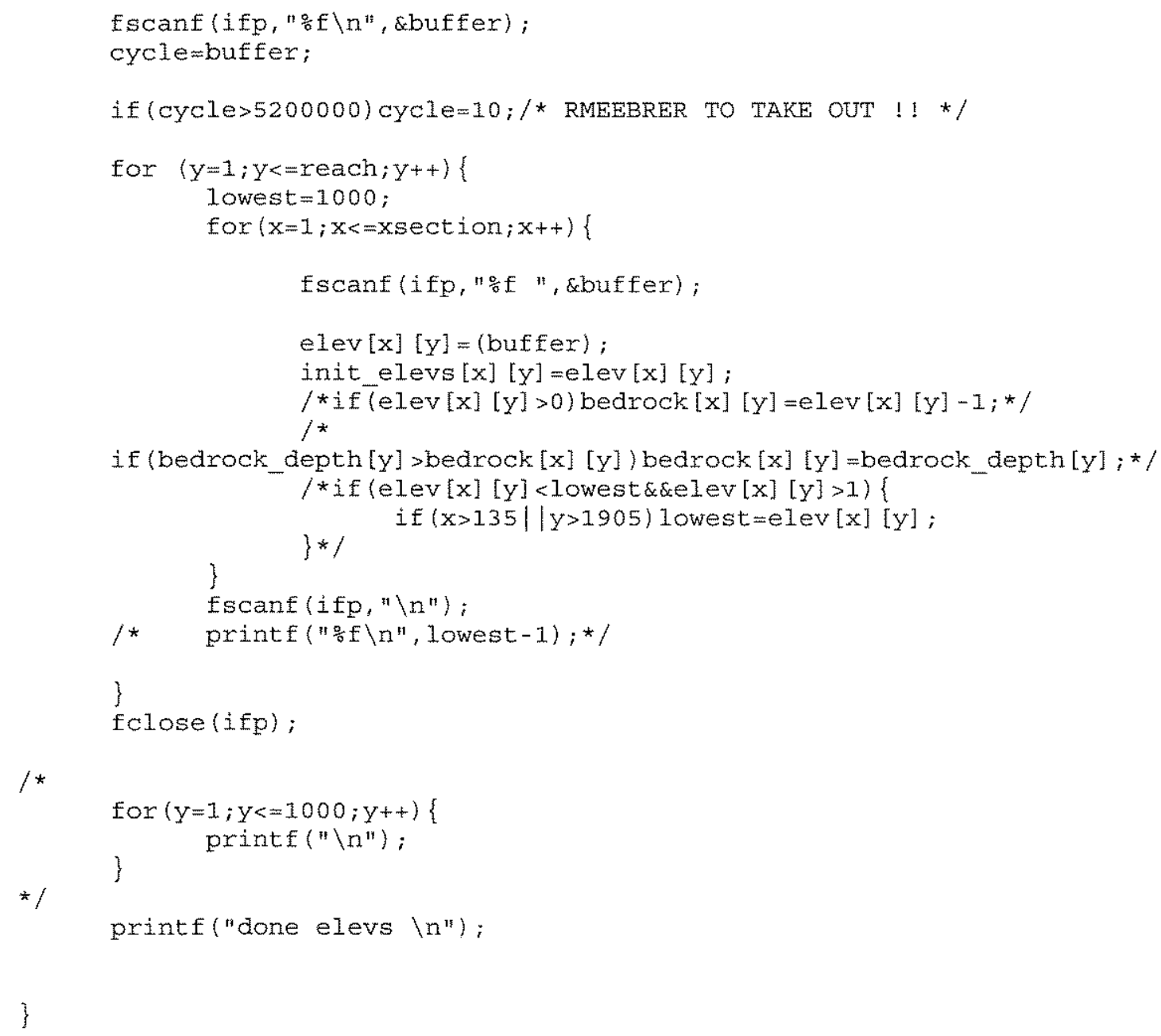

